\title{
Euler structures, the variety of representations and the Milnor-Turaev torsion
}

\author{
DAN BURGHELEA \\ STEFAN HALLER
}

\begin{abstract}
In this paper we extend and Poincare dualize the concept of Euler structures, introduced by Turaev for manifolds with vanishing Euler-Poincaré characteristic, to arbitrary manifolds. We use the Poincaré dual concept, co-Euler structures, to remove all geometric ambiguities from the Ray-Singer torsion by providing a slightly modified object which is a topological invariant. We show that when the co-Euler structure is integral then the modified Ray-Singer torsion when regarded as a function on the variety of generically acyclic complex representations of the fundamental group of the manifold is the absolute value of a rational function which we call in this paper the Milnor-Turaev torsion.
\end{abstract}

57R20; 58J52

\section{Introduction}

This paper was motivated by a question in geometric analysis.

Question Is the Ray-Singer torsion, when regarded as a positive real-valued function on the space of complex representations of the fundamental group, the absolute value of a holomorphic/meromorphic function. If so, what is the meaning of its argument? ${ }^{1}$

The answer provided in this paper goes as follows; cf Section 4.6. With the help of an integral co-Euler structure (for definitions and properties see Section 3.2) we decompose the Ray-Singer torsion as a product of two positive real-valued functions, the combinatorial torsion and the Bismut-Zhang anomaly. We verify that the first one, when restricted to generically acyclic representations, is the absolute value of a rational function, the Milnor-Turaev torsion, and the second is the absolute value of a holomorphic function.

\footnotetext{
${ }^{1}$ A similar question was treated by Quillen [26]. Instead of the variety of representation Quillen considers the affine space of complex structures in a smooth complex vector bundle over a Riemann surface, and instead of the Ray-Singer torsion the square root of the determinant of $D^{*} D$ where $D$ is the appropriate Cauchy-Riemann operator. More recently, a series of preprints of Braverman and Kappeler [3; $4 ; 5 ; 6]$ have been posted treating a similar question.
} 
The rational function is a topological invariant of the manifold and carries significant topological information; see Section 4.3. Its absolute value is a familiar quantity but the argument is an unexplored invariant which is analogous to the Atiyah-Patodi-Singer eta invariant [1], but not the same.

The values of the second function are obtained from an integral on a noncompact manifold, possibly divergent, but always regularizable and referred to as the invariant $R$ defined in Section 2.5. This invariant was first introduced by Bismut-Zhang and is a sort of Chern-Simons quantity. Both, the regularization of the integral and the structure of the invariant $R$, deserve and receive in this paper special attention.

The main results of the paper are contained in Theorem 3.10, Theorem 4.3, Theorem 4.9, Corollary 4.10 and Corollary 4.11. The paper contains a number of additional facts/results of independent interest expanding on the concepts described in the paper.

Let us make a more detailed presentation of the contents of this paper.

Section 2 is largely expository. We collect a number of facts about Riemannian metrics and vector fields with isolated zeros and about the Kamber-Tondeur form; see Section 2.1-Section 2.3. In Section 2.4 we describe the regularization of some possibly divergent integrals which is essential in the subsequent considerations (the invariant $R$, the comparison of Euler and co-Euler structures, the modifications of torsions). In Section 2.5 we define the invariant $R$.

We regard the invariant $R$ as providing a pairing between a Riemannian metric and a vector field with isolated zeros, with values in one-currents rel. boundary. This is in analogy with the Chern-Simons form which provides such pairing for two Riemannian metrics and with the Chern-Simons one-chain rel. boundary which provides a pairing between two vector fields with isolated zeros.

Section 3 discusses Euler and co-Euler structures and what they are good for. Turaev [31] introduced the concept of Euler structures for manifolds with vanishing Euler-Poincaré characteristics $\chi(M)$. It was observed by the first author [7] that Euler structures can be defined for manifolds with arbitrary Euler characteristics at the expense of a base point $x_{0} \in M$. The set of Euler structures $\mathfrak{E u l}_{x_{0}}(M ; \mathbb{Z})$ is an affine version of $H_{1}(M ; \mathbb{Z})$ in the sense that $H_{1}(M ; \mathbb{Z})$ acts freely and transitively on $\mathfrak{E u l}_{x_{0}}(M ; \mathbb{Z})$. There is also a real version of Euler structures which is an affine version of $H_{1}(M ; \mathbb{R})$ and will be denoted by $\mathfrak{E u l}_{x_{0}}(M ; \mathbb{R})$. There is a canonical map $\mathfrak{E} \mathfrak{u l}_{x_{0}}(M ; \mathbb{Z}) \rightarrow \mathfrak{E u l}_{x_{0}}(M ; \mathbb{R})$ which is affine over the homomorphism $H_{1}(M ; \mathbb{Z}) \rightarrow H_{1}(M ; \mathbb{R})$; see Section 3.1.

Given an Euler structure $\mathfrak{e} \in \mathfrak{E u l}_{x_{0}}(M ; \mathbb{Z})$, a cohomology orientation $\mathfrak{o}$, ie, an orientation of $\bigoplus_{i} H^{i}(M ; \mathbb{R})$, and a representation $\rho \in \operatorname{Rep}(\Gamma ; V), \Gamma=\pi_{1}\left(M, x_{0}\right)$, we use 
the Milnor-Turaev construction $[24 ; 31]$ to provide an element $\tau_{\text {comb }}^{\rho, \mathfrak{e}, \mathfrak{o}}$ in the determinant line $^{2}$

$$
\operatorname{Det}_{x_{0}}(M ; \rho):=\operatorname{det} H^{*}(M ; \rho) \otimes \operatorname{det} V^{-\chi(M)}
$$

which will be called Milnor-Turaev torsion; see Definition 3.12.

For the dependence on $\mathfrak{e}$ and $\mathfrak{o}$ we find

$$
\tau_{\mathrm{comb}}^{\rho, \mathfrak{e}+\sigma, \mathfrak{o}}=\tau_{\mathrm{comb}}^{\rho, \mathfrak{e}, \mathfrak{o}} \cdot[\operatorname{det} \circ \rho](\sigma)^{-1} \quad \text { and } \quad \tau_{\mathrm{comb}}^{\rho, \mathfrak{e},-\mathfrak{o}}=(-1)^{\operatorname{dim} V} \tau_{\mathrm{comb}}^{\rho, \mathfrak{e}, \mathfrak{o}}
$$

for all $\sigma \in H_{1}(M ; \mathbb{Z})$. Here $[\operatorname{det} \circ \rho]: H_{1}(M ; \mathbb{Z}) \rightarrow \mathbb{C}^{*}$ denotes the homomorphism induced from the homomorphism det $\circ \rho: \Gamma \rightarrow \mathbb{C}^{*}$.

If $H^{*}(M ; \rho)=0$ then $\operatorname{Det}_{x_{0}}(M ; \rho)=\mathbb{C}$, and $\tau_{\text {comb }}^{\rho, \mathfrak{e}, \mathfrak{o}}$ is a nonzero complex number.

In Section 3.2 we introduce the concept of co-Euler structures. The set of co-Euler structures $\mathfrak{E u l}_{x_{0}}^{*}(M ; \mathbb{R})$ is an affine version of $H^{n-1}\left(M ; \mathcal{O}_{M}\right)$ and depends on the choice of a base point $x_{0} \in M$ if $\chi(M) \neq 0$. By definition a co-Euler structure is an equivalence class of pairs $(g, \alpha)$, where $g$ is a Riemannian metric on $M$, and $\alpha \in \Omega^{n-1}\left(M \backslash\left\{x_{0}\right\} ; \mathcal{O}_{M}\right)$ with $d \alpha=E(g)$ where $E(g) \in \Omega^{n}\left(M ; \mathcal{O}_{M}\right)$ denotes the Euler form of $g$. Two such pairs $\left(g_{1}, \alpha_{1}\right)$ and $\left(g_{2}, \alpha_{2}\right)$ are equivalent if $\alpha_{2}-\alpha_{1}=$ $\operatorname{cs}\left(g_{1}, g_{2}\right)$ where $\operatorname{cs}\left(g_{1}, g_{2}\right) \in \Omega^{n-1}\left(M ; \mathcal{O}_{M}\right) / d \Omega^{n-2}\left(M ; \mathcal{O}_{M}\right)$ denotes the ChernSimons class. The main properties of Euler and co-Euler structures and the relationship between them are collected in Theorem 3.10. Co-Euler structures are nicely suited to remove the metric ambiguities of the analytic torsion and finally provide a topological invariant, the modified Ray-Singer metric/torsion.

More precisely, let $\mathbb{F}_{\rho}$ denote the flat complex vector bundle over $M$ associated to a representation $\rho \in \operatorname{Rep}(\Gamma ; V)$. Recall that the Ray-Singer metric is a Hermitian metric $\|\cdot\|_{\mathrm{RS}}^{\rho, g, \mu}$ on the determinant line $\operatorname{det} H^{*}\left(M ; \mathbb{F}_{\rho}\right)=\operatorname{det} H^{*}(M ; \rho)$ depending on a Riemannian metric $g$ on $M$ and a Hermitian structure $\mu$ on $\mathbb{F}_{\rho}$ when $M$ has even dimension, but is independent of them when $M$ is of odd dimension; see Bismut and Zhang [2]. Using a co-Euler structure $\mathfrak{e}^{*} \in \mathfrak{E u}_{x_{0}}^{*}(M ; \mathbb{R})$ permits to define a modified Ray-Singer metric $\|\cdot\|_{\mathrm{an}}^{\rho, \mathrm{e}^{*}}$ on the determinant line (1); see Definition 3.15. The anomaly formulas for the Ray-Singer torsion imply that this Hermitian metric indeed only depends on the representation $\rho$ and the co-Euler structure $\mathfrak{e}^{*}$. For the

\footnotetext{
${ }^{2}$ Here we use the following notation. For a finite-dimensional vector space $V$ we write det $V:=$ $\Lambda^{\operatorname{dim} V} V$ for its determinant line, a one-dimensional vector space. For a finite-dimensional graded vector space $W^{*}$ we define the (graded) determinant line by $\operatorname{det} W^{*}:=\operatorname{det} W^{\text {even }} \otimes\left(\operatorname{det} W^{\text {odd }}\right)^{*}$. Moreover, for a line $L$, ie, one-dimensional vector space, and $k \in \mathbb{N}$ we write $L^{k}:=L \otimes \cdots \otimes L$ and $L^{-k}:=\left(L^{k}\right)^{*}$. Then there are natural identifications $L^{k+l}=L^{k} \otimes L^{l}$ for all $k, l \in \mathbb{Z}$.
} 
dependence on $\mathfrak{e}^{*}$ we have

$$
\|\cdot\|_{\mathrm{an}}^{\rho, \mathrm{e}^{*}+\beta}=\|\cdot\|_{\mathrm{an}}^{\rho, \mathrm{e}^{*}} \cdot e^{\left\langle\log |[\operatorname{det} \circ \rho]|, \mathrm{PD}^{-1}(\beta)\right\rangle}
$$

for all $\beta \in H^{n-1}\left(M ; \mathcal{O}_{M}\right)$. Here $\log |[\operatorname{det} \circ \rho]| \in H^{1}(M ; \mathbb{R})$ denotes the class corresponding to the homomorphism $\log |[\operatorname{det} \circ \rho]|: H_{1}(M ; \mathbb{Z}) \rightarrow \mathbb{R}$, which is paired with the Poincaré dual $\mathrm{PD}^{-1}(\beta)$ of $\beta$.

There is a natural isomorphism $P: \mathfrak{E u l}_{x_{0}}(M ; \mathbb{R}) \rightarrow \mathfrak{E u l}_{x_{0}}^{*}(M ; \mathbb{R})$ which is affine over the Poincaré duality isomorphism PD: $H_{1}(M ; \mathbb{R}) \rightarrow H^{n-1}\left(M ; \mathcal{O}_{M}\right)$; see Section 3.2. We continue to denote by $P$ the composition of $P$ with the canonical map $\mathfrak{E u l}_{x_{0}}(M ; \mathbb{Z}) \rightarrow \mathfrak{E u r}_{x_{0}}^{*}(M ; \mathbb{R})$ and refer to $P\left(\mathfrak{E u l}_{x_{0}}(M ; \mathbb{Z})\right)$ as the lattice of integral co-Euler structures.

This permits a reformulation of the Bismut-Zhang theorem [2] as a statement which compares two topological invariants; see Theorem 3.17. More precisely, it says that analysis is able to recover the absolute value of the Milnor-Turaev torsion

$$
\left\|\tau_{\mathrm{comb}}^{\rho, \mathfrak{e}, \mathfrak{o}}\right\|_{\mathrm{an}}^{\rho, \mathrm{e}^{*}}=e^{\left\langle\log |[\operatorname{det} \circ \rho]|, \mathrm{PD}^{-1}\left(\mathrm{e}^{*}-P(\mathfrak{e})\right)\right\rangle} .
$$

Here $\log \mid[$ det $\circ \rho] \mid \in H^{1}(M ; \mathbb{R})$ denotes the cohomology class corresponding to the homomorphism $\log |[\operatorname{det} \circ \rho]|: H_{1}(M ; \mathbb{Z}) \rightarrow \mathbb{R}$.

Section 4 is dedicated to the Milnor-Turaev torsion viewed as a rational function on an algebraic set of generically acyclic representations which will be described below. This rational function is presented from a perspective analogous to the theory of characteristic classes of vector bundles (via the Grassmann variety, the Chern forms of the tautological bundle and the classifying map.)

Here is a brief description. For a collection $\left(k_{0}, k_{1}, \ldots, k_{n}\right)$ of nonnegative integers satisfying $k_{i}-k_{i-1} \pm \cdots+(-1)^{i} k_{0} \geq 0, i \leq n-1$ and $k_{n}-k_{n-1} \pm \cdots+(-1)^{n} k_{0}=0$, we denote by $\mathbb{D}_{\mathrm{ac}}\left(k_{0}, \ldots, k_{n}\right)$ the set of acyclic complexes $0 \rightarrow \mathbb{C}^{k_{0}} \rightarrow \cdots \rightarrow \mathbb{C}^{k_{n}} \rightarrow 0$. In Section 4.2 we will see that its Zariski closure is an (irreducible) affine variety. We will denote this closure by $\widehat{\mathbb{D}}_{\mathrm{ac}}\left(k_{0}, \ldots, k_{n}\right)$ and refer to it as the variety of generically acyclic complexes. The assignment which associates to an acyclic complex $C$ its torsion $\mathfrak{t}(C)$ defines a rational function $\mathfrak{t}$ on $\widehat{\mathbb{D}}_{\mathrm{ac}}\left(k_{0}, \ldots, k_{n}\right)$.

For a finitely presented group $\Gamma$ we denote by $\operatorname{Rep}(\Gamma ; V)$ the complex algebraic set of complex representations of $\Gamma$ on the complex vector space $V$; cf Section 4.1.

For a closed pointed manifold $\left(M, x_{0}\right)$ with $\Gamma=\pi_{1}\left(M, x_{0}\right)$ denote by $\operatorname{Rep}^{M}(\Gamma ; V)$ the algebraic closure of $\operatorname{Rep}_{0}^{M}(\Gamma ; V)$, that is, the Zariski open set of representations $\rho \in \operatorname{Rep}(\Gamma ; V)$ so that $H^{*}(M ; \rho)=0$. The elements of $\operatorname{Rep}^{M}(\Gamma ; V)$ are called generically acyclic representations. 
By choosing a triangulation $\tau$ of $M$, a collection of paths $\pi_{\tau}=\left\{\pi_{x_{\sigma}}\right\}$ from $x_{0}$ to the barycenters $x_{\sigma}$ of each simplex $\sigma$, an ordering $o$ of the set of simplices and a base (frame) $\epsilon$ of $V$, one obtains a regular map $t_{\pi_{\tau}, o, \epsilon}: \operatorname{Rep}^{M}(\Gamma ; V) \rightarrow \widehat{\mathbb{D}}_{\mathrm{ac}}\left(k_{0}, \ldots, k_{n}\right)$ with $k_{i}=\operatorname{dim}(V) \times \sharp\left(\mathcal{X}_{i}\right)$ where $\mathcal{X}_{i}$ denotes the set of $i$-simplices and $\sharp$ denotes cardinality. One can show that while the map $t_{\pi_{\tau}, o, \epsilon}$ depends on $\left(\pi_{\tau}, o, \epsilon\right)$, the pullback of $\mathfrak{t}$ by $t_{\pi_{\tau}, o, \epsilon}$ depends only on the Euler structure $\mathfrak{e}$ defined by $\left(\tau, \pi_{\tau}\right)$ (more precisely by $\left(X_{\tau}, \pi_{\tau}\right)$, cf Section 3.1), and the cohomology orientation $\mathfrak{o}$ induced by $o$. We denote the pullback $\mathfrak{t} \cdot t_{\pi_{\tau}, o, \epsilon}$ by

$$
\mathcal{T}_{\text {comb }}^{\mathfrak{e}, \mathcal{o}}: \operatorname{Rep}_{0}^{M}(\Gamma ; V) \rightarrow \mathbb{C}^{*} .
$$

In Section 4.2, in particular Theorem 4.3, we observe that this function is a rational function on $\operatorname{Rep}^{M}(\Gamma ; V)$ whose zeros and poles are therefore contained in the complement $\operatorname{Rep}^{M}(\Gamma ; V) \backslash \operatorname{Rep}_{o}^{M}(\Gamma ; V)$. Recall that for acyclic $\rho$ we have a canonical identification $\operatorname{Det}_{x_{0}}(M ; \rho)=\mathbb{C}$, and via this identification $\mathcal{T}_{\text {comb }}^{\mathfrak{e}, \mathfrak{o}}(\rho)=\left(\tau_{\text {comb }}^{\rho, \mathfrak{e}, \mathfrak{o}}\right)^{-1}$.

The function $\mathcal{T}_{\text {comb }}^{\mathfrak{e}, \mathfrak{o}}$ contains relevant topological information, even in the simplest possible examples. In Section 4.3 we compute this function for a mapping torus in terms of the Lefschetz zeta function of the gluing diffeomorphism.

If $M$ is obtained by surgery on a framed knot and $\operatorname{dim} V=1$, the function $\mathcal{T}_{\text {comb }}^{\mathfrak{e}, \mathfrak{o}}$ coincides with the Alexander polynomial of the knot; see Turaev [33].

If $\mathfrak{e}_{1}, \mathfrak{e}_{2} \in \mathfrak{E u l}_{x_{0}}(M ; \mathbb{Z})$ are two Euler structures with $P\left(\mathfrak{e}_{1}\right)=P\left(\mathfrak{e}_{2}\right)$ and $\mathfrak{o}_{1}, \mathfrak{o}_{2}$ are two cohomology orientations then the two functions $\mathcal{T}_{\text {comb }}^{\mathfrak{e}_{1}, \mathfrak{o}_{1}}$ and $\mathcal{T}_{\text {comb }}^{\mathfrak{e}_{2}, \mathfrak{o}_{2}}$ differ by multiplication with a root of unity; see (2). Therefore, up to multiplication by a root of unity, $\mathcal{T}_{\text {comb }}^{\mathfrak{e}, \mathfrak{o}}$ depends only on $\mathfrak{e}^{*}=P(\mathfrak{e})$. The Bismut-Zhang theorem implies that the absolute value of $\mathcal{T}_{\text {comb }}^{\mathfrak{e}, \mathfrak{a}}$ actually coincides with the modified Ray-Singer torsion associated with $\mathfrak{e}^{*}$, ie, the quantity

$$
\mathcal{T}_{\text {an }}^{\mathfrak{e}^{*}}(\rho)=T_{\text {an }}\left(\nabla_{\rho}, g, \mu\right) \cdot e^{-\int_{M} \omega\left(\nabla_{\rho}, \mu\right) \wedge \alpha} .
$$

Here $\mathbb{F}_{\rho}=\left(F_{\rho}, \nabla_{\rho}\right)$ denotes the flat complex vector bundle associated to the representation $\rho \in \operatorname{Rep}_{o}^{M}(\Gamma ; V), g$ is a Riemannian metric on $M, \mu$ is a Hermitian structure on $F_{\rho}, T_{\text {an }}\left(\nabla_{\rho}, g, \mu\right)$ denotes the Ray-Singer torsion, $\omega\left(\nabla_{\rho}, \mu\right)$ is the Kamber-Tondeur form (Section 2.3) and $\alpha$ is such that the pair $(g, \alpha)$ represents the co-Euler structure $\mathfrak{e}^{*}$. Recall that for acyclic $\rho$ we have a canonical identification $\operatorname{Det}_{x_{0}}(M ; \rho)=\mathbb{C}$ and via this identification $\mathcal{T}_{\text {an }}^{\mathfrak{e}^{*}}(\rho)=\|1\|_{\text {an }}^{\rho, \mathfrak{e}^{*}}$.

We use this fact to conclude (under some additional hypotheses) that the Ray-Singer torsion, when viewed as a function on the analytic set of flat connections, is the absolute value of a meromorphic function. The precise statement is contained in Corollary 4.10. This partially answers the question formulated at the beginning of this introduction. 
This was also known to $\mathrm{W}$ Müller at least restricting to unimodular flat connections. A more analytic treatment is announced in $[11 ; 10 ; 9]$; see also $[6 ; 4 ; 5 ; 3]$.

The argument of the complex number $\mathcal{T}_{\text {comb }}^{\mathfrak{e}, \mathfrak{o}}(\rho)$ is an apparently unexplored invariant. If $U$ is an open neighborhood of $[1,2] \times\{0\} \subseteq \mathbb{C}$ and $\rho: U \rightarrow \operatorname{Rep}_{0}^{M}(\Gamma ; V)$ is a holomorphic path in the nonsingular part of $\operatorname{Rep}_{0}^{M}(\Gamma ; V)$ joining $\rho_{1}=\rho(1)$ with $\rho_{2}=\rho(2)$, then the difference

$$
\arg \mathcal{T}_{\text {comb }}^{\mathfrak{e}, \mathfrak{o}}\left(\rho_{2}\right)-\arg \mathcal{T}_{\text {comb }}^{\mathfrak{e}, \mathfrak{o}}\left(\rho_{1}\right) \in \mathbb{R} / 2 \pi \mathbb{Z}
$$

can be derived from $\mathcal{T}_{\text {an }}^{\mathfrak{e}^{*}}(\rho(z)), P(\mathfrak{e})=\mathfrak{e}^{*}$; see (61) in Section 4.4. In general two representations $\rho_{1}$ and $\rho_{2}$ in the same connected component of the nonsingular part of $\operatorname{Rep}_{0}^{M}(\Gamma ; V)$ cannot be joined by a holomorphic path. However, one can always find a finite collection of representations $\rho^{i} \in \operatorname{Rep}_{0}^{M}(\Gamma ; V), i=1, \ldots, k$, with $\rho^{1}=\rho_{1}$ and

$\rho^{k}=\rho_{2}$ so that holomorphic paths from $\rho^{i}$ to $\rho^{i+1}$ do exist, and implicitly benefit from such formula.

As another application we provide a derivation of Marcsik's theorem (unpublished [22]) from the Bismut-Zhang theorem and the computation of $\mathcal{T}_{\text {comb }}^{\mathfrak{e}, \mathfrak{o}}$ for mapping tori; see Section 4.5.

Acknowledgements We would like to thank V Turaev for pointing out a sign mistake and some lacking references in a previous version of the paper. We also would like to thank the referee for suggestions and requests of clarifications. We believe they have improved the quality of the exposition. Part of this work was done while both authors enjoyed the hospitality of the Max Planck Institute for Mathematics in Bonn. A previous version was written while the second author enjoyed the hospitality of the Ohio State University. The second author was partially supported by the Fonds zur Förderung der wissenschaftlichen Forschung (Austrian Science Fund), project number P14195-MAT.

\section{A few characteristic forms and the invariant $R$}

\subsection{Euler, Chern-Simons, and the Mathai-Quillen form}

In this section we will briefly recall basic properties of the Euler form, the MathaiQuillen form and the Chern-Simons class. For more details we refer to [23] or [2, section III]. Although these forms are defined for any real vector bundle equipped with a connection and a parallel Hermitian metric we will only discuss them for the tangent bundle of a Riemannian manifold and the Levi-Civita connection. 
Let $M$ be smooth closed manifold of dimension $n$. Let $\pi: T M \rightarrow M$ denote the tangent bundle and $\mathcal{O}_{M}$ the orientation bundle, a flat real line bundle over $M$. For a Riemannian metric $g$ denote its Euler form by

$$
E(g) \in \Omega^{n}\left(M ; \mathcal{O}_{M}\right)
$$

and by

$$
\Psi(g) \in \Omega^{n-1}\left(T M \backslash M ; \pi^{*} \mathcal{O}_{M}\right)
$$

its Mathai-Quillen form. For two Riemannian metrics $g_{1}$ and $g_{2}$ we let

$$
\operatorname{cs}\left(g_{1}, g_{2}\right) \in \Omega^{n-1}\left(M ; \mathcal{O}_{M}\right) / d\left(\Omega^{n-2}\left(M ; \mathcal{O}_{M}\right)\right)
$$

denote their Chern-Simons class. The following relations hold:

$$
\begin{aligned}
d \operatorname{cs}\left(g_{1}, g_{2}\right) & =E\left(g_{2}\right)-E\left(g_{1}\right) \\
\operatorname{cs}\left(g_{2}, g_{1}\right) & =-\operatorname{cs}\left(g_{1}, g_{2}\right) \\
\operatorname{cs}\left(g_{1}, g_{3}\right) & =\operatorname{cs}\left(g_{1}, g_{2}\right)+\operatorname{cs}\left(g_{2}, g_{3}\right) \\
E(g) & =(-1)^{n} E(g) \\
\operatorname{cs}\left(g_{1}, g_{2}\right) & =(-1)^{n} \operatorname{cs}\left(g_{1}, g_{2}\right)
\end{aligned}
$$

When $n$ is odd both, the Euler form and the Chern-Simons class, vanish. Moreover, we have the equalities

$$
\begin{aligned}
d \Psi(g) & =\pi^{*} E(g) \\
\Psi\left(g_{2}\right)-\Psi\left(g_{1}\right) & \equiv \pi^{*} \operatorname{cs}\left(g_{1}, g_{2}\right) \quad \bmod d \Omega^{n-2}\left(T M \backslash M ; \pi^{*} \mathcal{O}_{M}\right) \\
v^{*} \Psi(g) & =(-1)^{n} \Psi(g)
\end{aligned}
$$

where $v: T M \rightarrow T M$ denotes the canonical involution, $v(x):=-x$.

For $M=\mathbb{R}^{n}$ equipped with the standard Riemannian metric $g_{0}$ we have

$$
\Psi\left(g_{0}\right)=\frac{\Gamma(n / 2)}{(2 \pi)^{n / 2}} \sum_{i=1}^{n}(-1)^{i} \frac{\xi_{i}}{\left(\sum \xi_{i}^{2}\right)^{n / 2}} d \xi_{1} \wedge \cdots \wedge \widehat{d \xi_{i}} \wedge \cdots \wedge d \xi_{n}
$$

in standard coordinates $x_{1}, \ldots, x_{n}, \xi_{1}, \ldots, \xi_{n}$ on $T M$. In general, the restriction of $\Psi$ to a fiber $T_{X} M$ coincides with the negative of the standard generator of the group $H^{n-1}\left(T_{x} M \backslash 0 ; \mathcal{O}_{T_{x} M}\right)$.

Suppose $X$ is a vector field with isolated zero $x$, and let $B_{\epsilon}(x)$ denote the ball of radius $\epsilon$ centered at $x$, with respect to some chart. From (9) and (11) one easily deduces

$$
\lim _{\epsilon \rightarrow 0} \int_{\partial\left(M \backslash B_{\epsilon}(x)\right)} X^{*} \Psi(g)=\operatorname{IND}_{X}(x),
$$


where $\operatorname{IND}_{X}(x)$ denotes the Hopf index of $X$ at $x$.

\subsection{Euler and Chern-Simons class for vector fields}

Let $M$ be a closed manifold and suppose $X$ is a vector field with isolated set of zeros $\mathcal{X} \subseteq M$. For every $x \in \mathcal{X}$ we have a Hopf index $\operatorname{IND}_{X}(x)$ of $X$ at $x$. Define a singular ${ }^{3}$ zero-chain

$$
E(X):=\sum_{x \in \mathcal{X}} \operatorname{IND}_{X}(x) x \in C_{0}(M ; \mathbb{Z})
$$

Given two vector fields $X_{1}$ and $X_{2}$ we are going to define a singular one-chain, well-defined up to boundaries,

$$
\operatorname{cs}\left(X_{1}, X_{2}\right) \in C_{1}(M ; \mathbb{Z}) / \partial C_{2}(M ; \mathbb{Z})
$$

with the properties:

$$
\begin{aligned}
\partial \operatorname{cs}\left(X_{1}, X_{2}\right) & =E\left(X_{2}\right)-E\left(X_{1}\right) \\
\operatorname{cs}\left(X_{2}, X_{1}\right) & =-\operatorname{cs}\left(X_{1}, X_{2}\right) \\
\operatorname{cs}\left(X_{1}, X_{3}\right) & =\operatorname{cs}\left(X_{1}, X_{2}\right)+\operatorname{cs}\left(X_{2}, X_{3}\right) \\
E(-X) & =(-1)^{n} E(X) \\
\operatorname{cs}\left(-X_{1},-X_{2}\right) & =(-1)^{n} \operatorname{cs}\left(X_{1}, X_{2}\right)
\end{aligned}
$$

This should be compared with the equalities (3)-(7) in Section 2.1.

Equation (17) follows from $\operatorname{IND}_{-X}(x)=(-1)^{n} \operatorname{IND}_{X}(x)$. The construction of (13) is accomplished by first reducing to vector fields with nondegenerate zeros with the help of a small perturbation, and then taking the zero set of a generic homotopy connecting $X_{1}$ with $X_{2}$.

More precisely, suppose we have two vector fields $X_{1}^{\prime}$ and $X_{2}^{\prime}$ with nondegenerate zeros. Consider the vector bundle $p^{*} T M \rightarrow I \times M$, where $I$ is the interval $[1,2]$ and $p: I \times$ $M \rightarrow M$ denotes the natural projection. Choose a section $\mathbb{X}$ of $p^{*} T M$ transversal to the zero section and which restricts to $X_{i}^{\prime}$ on $\{i\} \times M, i=1,2$. The zero set of $\mathbb{X}$ is a canonically oriented one-dimensional submanifold with boundary. Its fundamental class, when pushed forward via $p$, gives rise to $c(\mathbb{X}) \in C_{1}(M ; \mathbb{Z}) / \partial C_{2}(M ; \mathbb{Z})$ satisfying (14).

Suppose $\mathbb{X}_{1}$ and $\mathbb{X}_{2}$ are two nondegenerate homotopies from $X_{1}^{\prime}$ to $X_{2}^{\prime}$. Then we have $c\left(\mathbb{X}_{1}\right)=c\left(\mathbb{X}_{2}\right) \in C_{1}(M ; \mathbb{Z}) / \partial C_{2}(M ; \mathbb{Z})$. Indeed, consider the vector bundle $q^{*} T M \rightarrow I \times I \times M$, where $q: I \times I \times M \rightarrow M$ denotes the natural projection.

\footnotetext{
${ }^{3}$ Here, and throughout the paper, singular chain stands for smooth singular chain.
} 
Choose a section of $q^{*} T M$ which is transversal to the zero section, restricts to $\mathbb{X}_{i}$ on $\{i\} \times I \times M, i=1,2$, and restricts to $X_{i}^{\prime}$ on $\{s\} \times\{i\} \times M$ for all $s \in I$ and all $i=1,2$. The zero set of such a section then gives rise to $\sigma$ satisfying $c\left(\mathbb{X}_{2}\right)-c\left(\mathbb{X}_{1}\right)=\partial \sigma$. So we get a well-defined $\operatorname{cs}\left(X_{1}^{\prime}, X_{2}^{\prime}\right):=c(\mathbb{X}) \in C_{1}(M ; \mathbb{Z}) / \partial C_{2}(M ; \mathbb{Z})$ satisfying (14), provided $X_{1}^{\prime}$ and $X_{2}^{\prime}$ have nondegenerate zero set.

Now suppose $X$ is a vector field with isolated zeros. For every zero $x \in \mathcal{X}$ we choose a contractible open neighborhood $B_{x}$ of $x$, assuming all $B_{x}$ are disjoint. Set $B:=\bigcup_{x \in \mathcal{X}} B_{x}$. Choose a vector field with nondegenerate zeros $X^{\prime}$ that coincides with $X$ on $M \backslash B$. Let $\mathcal{X}^{\prime}$ denote its zero set. Then $\operatorname{IND}_{X}(x)=\sum_{y \in \mathcal{X}^{\prime} \cap B_{x}} \operatorname{IND}_{X^{\prime}}(y)$ for every $x \in \mathcal{X}$. So we can choose a one-chain $c\left(X, X^{\prime}\right)$ in $B$ which satisfies $\partial c\left(X, X^{\prime}\right)=E\left(X^{\prime}\right)-E(X)$. Since $H_{1}(B ; \mathbb{Z})$ vanishes, the one-chain $c\left(X, X^{\prime}\right)$ is well-defined up to a boundary.

Given two vector fields $X_{1}$ and $X_{2}$ with isolated zeros we choose $B, X_{1}^{\prime}, X_{2}^{\prime}$, $c\left(X_{1}, X_{1}^{\prime}\right)$ and $c\left(X_{2}, X_{2}^{\prime}\right)$ as above and set

$$
\operatorname{cs}\left(X_{1}, X_{2}\right):=c\left(X_{1}, X_{1}^{\prime}\right)+\operatorname{cs}\left(X_{1}^{\prime}, X_{2}^{\prime}\right)-c\left(X_{2}, X_{2}^{\prime}\right) .
$$

Note that for two vector fields which coincide on $M \backslash B$ and have nondegenerate zeros only, one can construct a nondegenerate homotopy between them which is constant on $M \backslash B$. Together with $H_{1}(B ; \mathbb{Z})=0$ this immediately implies that the one-chain $\operatorname{cs}\left(X_{1}, X_{2}\right) \in C_{1}(M ; \mathbb{Z}) / \partial C_{2}(M ; \mathbb{Z})$ is independent of these choices. Clearly (14)-(16) and (18) hold too.

\subsection{Kamber-Tondeur one-form}

Let $E$ be a real or complex vector bundle over $M$. For a connection $\nabla$ and a Hermitian structure $\mu$ on $E$ we define a real-valued one-form $\omega(\nabla, \mu):=-\frac{1}{2} \operatorname{tr}_{\mu}(\nabla \mu)$ in $\Omega^{1}(M ; \mathbb{R})$. More explicitly, for a tangent vector $X \in T M$

$$
\omega(\nabla, \mu)(X):=-\frac{1}{2} \operatorname{tr}_{\mu}\left(\nabla_{X} \mu\right) .
$$

This form is known as Kamber-Tondeur form [20]. Our convention differs from the one in Bismut and Zhang [2] by a factor $-\frac{1}{2}$. Here one interprets $\mu$ as a section in the bundle $H$ of sesquilinear forms on $E$ equipped with the induced connection (still denoted by $\nabla$ ) and one denotes by $\operatorname{tr}_{\mu}$ the bundle map from $H$ to the trivial rank one bundle defined by $\mu$. Note that if $\nabla$ is flat then $\omega(\nabla, \mu)$ will be a closed one-form. This and the following formulas are easily seen by noticing that $\omega\left(\nabla^{\operatorname{det} E}, \mu^{\operatorname{det} E}\right)=\omega(\nabla, \mu)$ where $\nabla^{\operatorname{det} E}$ and $\mu^{\operatorname{det} E}$ denote the induced connection and Hermitian structure on the determinant line $\operatorname{det} E:=\Lambda^{\operatorname{rank} E} E$. The verification of this equality is straightforward. One calculates $\omega$ in coordinates and with respect to a $\mu$-orthonormal frame in the 
neighborhood of a given point $x \in M$. A simple comparison between the two sides of the equality above verifies the statement.

Recall that the space of connections on $E$ is an affine space over $\Omega^{1}\left(M ; E^{*} \otimes E\right)$. For two connections $\nabla^{1}$ and $\nabla^{2}$ we find

$$
\omega\left(\nabla^{2}, \mu\right)-\omega\left(\nabla^{1}, \mu\right)=\mathfrak{R}\left(\operatorname{tr}\left(\nabla^{2}-\nabla^{1}\right)\right)
$$

where the trace is with respect to the last two variables, and $\mathfrak{R}$ denotes the real part.

If $\mu_{1}$ and $\mu_{2}$ are Hermitian structures then there is a unique section $A \in C^{\infty}\left(E^{*} \otimes E\right)$ such that $\mu_{2}(e, f)=\mu_{1}(A e, f)$. We set $V\left(\mu_{1}, \mu_{2}\right):=|\operatorname{det} A|$ which is a positive real-valued function on $M$. Its value at $x \in M$ is the volume, with respect to $\left(\mu_{2}\right)_{x}$, of a parallelepiped obtained from an orthonormal base, with respect to $\left(\mu_{1}\right)_{x}$. We have

$$
\omega\left(\nabla, \mu_{2}\right)-\omega\left(\nabla, \mu_{1}\right)=-\frac{1}{2} d \log V\left(\mu_{1}, \mu_{2}\right)
$$

Suppose $\nabla$ is flat, and let $U \subseteq M$ be a contractible open set. Then we find a parallel Hermitian structure $\tilde{\mu}$ over $U$, and can use (20) to obtain a local primitive of the closed one-form $\left.\omega(\nabla, \mu)\right|_{U}=d\left(-\frac{1}{2} \log V(\tilde{\mu}, \mu)\right)$.

Remark 2.1 Suppose $\nabla$ is flat, and let $x_{0}$ be a base point in $M$. Parallel transport defines a right action of $\pi_{1}\left(M, x_{0}\right)$ on $E_{x_{0}}$, the fiber over $x_{0}$. Composing with the inversion we obtain a representation $\rho: \pi_{1}\left(M, x_{0}\right) \rightarrow \operatorname{GL}\left(E_{x_{0}}\right)$. Denote by $[$ det $\circ \rho]: H_{1}(M ; \mathbb{Z}) \rightarrow \mathbb{C}^{*}$ the homomorphism induced by the homomorphism det $\circ \rho: \pi_{1}\left(M, x_{0}\right) \rightarrow \mathbb{C}^{*}$. Let $\log |[\operatorname{det} \circ \rho]| \in H^{1}(M ; \mathbb{R})$ denote the cohomology class corresponding to the homomorphism $\log |[\operatorname{det} \circ \rho]|: H_{1}(M ; \mathbb{Z}) \rightarrow \mathbb{R}$. This class is called the Kamber-Tondeur class and is represented by the Kamber-Tondeur form $\omega(\nabla, \mu)$ for every Hermitian metric $\mu$. Put another way, the cohomology class of $\omega(\nabla, \mu)$ captures the absolute value of the holonomy in $\left(\operatorname{det} E, \nabla^{\operatorname{det} E}\right)$.

The following observation will be useful in the proof of Corollary 4.10.

Proposition 2.2 Let $E$ be a complex vector bundle over $M$ equipped with a Hermitian structure $\mu$. Then there exist mappings $\nabla \mapsto \widetilde{\omega}(\nabla, \mu)$ which are affine over the map $\operatorname{tr}: \Omega^{1}\left(M ; E^{*} \otimes E\right) \rightarrow \Omega^{1}(M ; \mathbb{C})$ such that $\Re(\widetilde{\omega}(\nabla, \mu))=\omega(\nabla, \mu)$ and such that $\tilde{\omega}(\nabla, \mu)$ is closed whenever $\nabla$ is flat.

Proof Choose a reference connection $\nabla^{\prime}$ on $E$, and define

$$
\tilde{\omega}(\nabla, \mu):=\operatorname{tr}\left(\nabla-\nabla^{\prime}\right)+\omega\left(\nabla^{\prime}, \mu\right) .
$$


This is certainly affine over the trace, and its real part coincides with $\omega(\nabla, \mu)$ in view of (19). If $E$ admits flat connections we can choose $\nabla^{\prime}$ to be flat. Then $\widetilde{\omega}(\nabla, \mu)$ will be closed provided $\nabla$ was flat.

\subsection{Regularization of an integral}

Let $M$ be a closed manifold of dimension $n$. Suppose $\alpha \in \Omega^{n-1}\left(M \backslash \mathcal{X} ; \mathcal{O}_{M}\right)$ is a form with isolated set of singularities $\mathcal{X} \subseteq M$, and suppose $d \alpha \in \Omega^{n}\left(M ; \mathcal{O}_{M}\right)$ is globally smooth. Here $\mathcal{O}_{M}$ denotes the orientation bundle of $M$, a flat real line bundle over $M$. For $x \in \mathcal{X}$ define a real number

$$
\operatorname{IND}_{\alpha}(x):=\lim _{\epsilon \rightarrow 0} \int_{\partial\left(M \backslash B_{\epsilon}(x)\right)} \alpha=\int_{\partial\left(M \backslash B_{\epsilon^{\prime}}(x)\right)} \alpha+\int_{B_{\epsilon^{\prime}}(x)} d \alpha
$$

where $B_{\epsilon}(x)$ denotes the ball of radius $\epsilon$ in a chart centered at $x$ and $\epsilon^{\prime}$ is small enough. Note that for a Riemannian metric $g$, and a vector field $X$ with isolated zero $x$, the form $X^{*} \Psi(g)$ has an isolated singularity at $x, d X^{*} \Psi(g)=E(g)$ is globally smooth, see (8), and in view of (12) we have

$$
\operatorname{IND}_{X}^{* \Psi(g)}(x)=\operatorname{IND}_{X}(x) .
$$

Let $\alpha$ be as above, and suppose $\omega \in \Omega^{1}(M)$ is a closed real or complex valued oneform. Choose a function $f$ such that $f$ vanishes on $\mathcal{X}$ and such that $\omega^{\prime}:=\omega-d f$ vanishes locally around $\mathcal{X}$. Define

$$
S(\omega, \alpha):=S(\omega, \alpha ; f):=\int_{M \backslash \mathcal{X}} \omega^{\prime} \wedge \alpha-\int_{M} f d \alpha .
$$

The notation is justified by the following lemma.

Lemma 2.3 The quantity $S(\omega, \alpha ; f)$ does not depend on the choice of $f$. It is linear in $\omega$ and $\alpha$. It satisfies:

(i) $S(d h, \alpha)=\sum_{x \in \mathcal{X}} h(x) \operatorname{IND}_{\alpha}(x)-\int_{M} h d \alpha$ for every smooth function $h$.

(ii) $S(\omega, \beta)=\int_{M} \omega \wedge \beta$ for all $\beta \in \Omega^{n-1}\left(M ; \mathcal{O}_{M}\right)$. Particularly, $S(\omega, d \gamma)=0$ for all $\gamma \in \Omega^{n-2}\left(M ; \mathcal{O}_{M}\right)$.

Proof Let us first show that $S(\omega, \alpha ; f)$ does not depend on $f$. So suppose $f_{i}$ are functions which vanish on $\mathcal{X}$ and are such that $\omega_{i}^{\prime}:=\omega-d f_{i}$ vanishes locally around $\mathcal{X}$. Then $\omega_{2}^{\prime}-\omega_{1}^{\prime}=-d\left(f_{2}-f_{1}\right)$ and $f_{2}-f_{1}$ vanish locally around $\mathcal{X}$. Using Stokes' theorem for forms with compact support on $M \backslash \mathcal{X}$ we get

$$
S\left(\omega, \alpha ; f_{2}\right)-S\left(\omega, \alpha ; f_{1}\right)=-\int_{M \backslash \mathcal{X}} d\left(f_{2}-f_{1}\right) \wedge \alpha-\int_{M}\left(f_{2}-f_{1}\right) d \alpha=0 .
$$


Now let us turn to (i). Choose a function $f$ so that $d h-d f$ vanishes locally around $\mathcal{X}$ and so that $f$ vanishes on $\mathcal{X}$. For every $x \in \mathcal{X}$ choose an embedded ball $B_{\epsilon}(x)$ around $x$ such that $d h-d f$ vanishes on $B_{\epsilon}(x)$. Then $h-f$ is constant equal to $h(x)$ on $B_{\epsilon}(x)$, and Stokes' theorem implies

$$
S(d h, \alpha)=\int_{M \backslash \mathcal{X}}(d h-d f) \wedge \alpha-\int_{M} f d \alpha=\sum_{x \in \mathcal{X}} h(x) \int_{\partial\left(M \backslash B_{\epsilon}(x)\right)} \alpha-\int_{M} h d \alpha .
$$

With $\epsilon \rightarrow 0$ the statement follows. The remaining assertions are obvious.

\subsection{The invariant $R$}

Let $M$ be a closed manifold of dimension $n$. For a closed one-form $\omega$ and two Riemannian metrics $g_{1}$ and $g_{2}$ we set

$$
R\left(\omega, g_{1}, g_{2}\right):=\int_{M} \omega \wedge \operatorname{cs}\left(g_{1}, g_{2}\right)
$$

Although $\operatorname{cs}\left(g_{1}, g_{2}\right)$ (Section 2.1), is only defined up to $d \Omega^{n-2}\left(M ; \mathcal{O}_{M}\right)$ the quantity (22) is unambiguously defined according to Stokes' theorem. If the dimension of $M$ is odd then $R\left(\omega, g_{1}, g_{2}\right)=0$; see (7).

For a closed one-form $\omega$, and two vector fields $X_{1}$ and $X_{2}$ with isolated zeros we set

$$
R\left(\omega, X_{1}, X_{2}\right):=\int_{\operatorname{cs}\left(X_{1}, X_{2}\right)} \omega .
$$

Again, even though $\operatorname{cs}\left(X_{1}, X_{2}\right)$ is only defined up to a boundary (Section 2.2) the quantity (23) is well-defined in view of Stokes' theorem.

For a Riemannian metric $g$ and a vector field $X$ with isolated zeros $\mathcal{X} \subseteq M$ we have $X^{*} \Psi(g) \in \Omega^{n-1}\left(M \backslash \mathcal{X} ; \mathcal{O}_{M}\right)$ and $d X^{*} \Psi(g)=E(g)$; see Section 2.1, especially (8). So we may define

$$
R(\omega, X, g):=S\left(\omega, X^{*} \Psi(g)\right)
$$

for every closed one-form $\omega$; see Section 2.4. The invariant $R(\omega, X, g)$ has first appeared in the work of Bismut and Zhang [2].

Proposition 2.4 On $M$, let $\omega$ be a closed one-form, $h$ a smooth function, $g$ and $g_{i}$ Riemannian metrics, and let $X$ and $X_{i}$ be vector fields with isolated zeros. Then:

(i) $R\left(\omega, g_{1}, g_{2}\right), R\left(\omega, X_{1}, X_{2}\right)$ and $R(\omega, X, g)$ are linear in $\omega$.

(ii) $R\left(\omega, g_{2}, g_{1}\right)=-R\left(\omega, g_{1}, g_{2}\right)$.

(iii) $R\left(\omega, X_{2}, X_{1}\right)=-R\left(\omega, X_{1}, X_{2}\right)$. 
(iv) $R(d h, X, g)=h(E(X))-\int_{M} h E(g)$.

(v) $R\left(d h, g_{1}, g_{2}\right)=\int_{M} h E\left(g_{1}\right)-\int_{M} h E\left(g_{2}\right)$.

(vi) $R\left(d h, X_{1}, X_{2}\right)=h\left(E\left(X_{2}\right)\right)-h\left(E\left(X_{1}\right)\right)$.

(vii) $R\left(\omega, X_{1}, X_{3}\right)=R\left(\omega, X_{1}, X_{2}\right)+R\left(\omega, X_{2}, X_{3}\right)$.

(viii) $R\left(\omega, g_{1}, g_{3}\right)=R\left(\omega, g_{1}, g_{2}\right)+R\left(\omega, g_{2}, g_{3}\right)$.

(ix) $R\left(\omega, X, g_{2}\right)-R\left(\omega, X, g_{1}\right)=R\left(\omega, g_{1}, g_{2}\right)$.

(x) $R\left(\omega, X_{2}, g\right)-R\left(\omega, X_{1}, g\right)=R\left(\omega, X_{1}, X_{2}\right)$.

Here $h(E(X))=\sum_{x \in \mathcal{X}} h(x) \operatorname{IND}_{X}(x)$ where $\mathcal{X}$ denotes the zero set of $X$.

Proof Statements (i)-(iii) and (v)-(viii) follow immediately from Stokes' theorem, Lemma 2.3, equations (3)-(5) and equations (14)-(16).

Assertion (iv) follows from Lemma 2.3(i), equation (21), and from $d X^{*} \Psi(g)=E(g)$; see (8).

Statement (ix) follows from Lemma 2.3(ii) and $X^{*} \Psi\left(g_{2}\right)-X^{*} \Psi\left(g_{1}\right)=\operatorname{cs}\left(g_{1}, g_{2}\right)$; see (9). Statements (iv) and (ix) can be also found in [2].

It remains to prove (x). In view of (iv) and (vi) we may assume that $\omega$ vanishes in a neighborhood of the zero set $\mathcal{X}_{1} \cup \mathcal{X}_{2}$. It is then clear that we may also assume that $X_{1}$ and $X_{2}$ have nondegenerate zeros only. Choose a nondegenerate homotopy $\mathbb{X}$ from $X_{1}$ to $X_{2}$. Perturbing the homotopy slightly, cutting it into several pieces and using (vii) we may assume that the zero set $\mathbb{X}^{-1}(0) \subseteq I \times M$ is actually contained in a closed simply connected $I \times V$. Again, we may assume that $\omega$ vanishes on $V$. Then obviously $R\left(\omega, X_{1}, X_{2}\right)=0$. Moreover, in this situation Stokes' theorem implies

$$
\begin{aligned}
R\left(\omega, X_{2}, g\right)-R\left(\omega, X_{1}, g\right) & =\int_{M \backslash V} \omega \wedge X_{2}^{*} \Psi(g)-\int_{M \backslash V} \omega \wedge X_{1}^{*} \Psi(g) \\
& =\int_{I \times(M \backslash V)} d\left(p^{*} \omega \wedge \mathbb{X}^{*} \tilde{p}^{*} \Psi(g)\right) \\
& =-\int_{I \times(M \backslash V)} p^{*}(\omega \wedge E(g))=0 .
\end{aligned}
$$

where $p: I \times M \rightarrow M$ denotes the natural projection, and $\tilde{p}: p^{*} T M \rightarrow T M$ denotes the natural vector bundle homomorphism over $p$. For the last calculation note that $d \mathbb{X}^{*} \tilde{p}^{*} \Psi(g)=p^{*} E(g)$ in view of (8), and that $\omega \wedge E(g)=0$ because of dimensional reasons.

Remark 2.5 A similar definition of $R(\omega, X, g)$ works for any vector field $X$ with arbitrary zero set $\mathcal{X}:=\{x \in M \mid X(x)=0\}$ provided $\omega$ is exact when restricted to a sufficiently small neighborhood of $\mathcal{X}$. 


\subsection{Extension of Chern-Simons theory}

The material in this section will not be used in the rest of the paper, but puts the observations in Proposition 2.4 in a more natural perspective.

Let $M$ be a closed manifold of dimension $n$. Equip $\Omega^{k}(M ; \mathbb{R})$ with the $C^{\infty}$-topology. The continuous linear functionals on $\Omega^{k}(M ; \mathbb{R})$ are called $k$-currents. Let $\mathcal{D}_{k}(M)$ denote the space of all $k$-currents and $\delta: \mathcal{D}_{k}(M) \rightarrow \mathcal{D}_{k-1}(M)$ be given by

$$
(\delta \varphi)(\alpha):=\varphi(d \alpha) .
$$

Clearly $\delta^{2}=0$. By the deRham theorem for currents the chain complex $\left(\mathcal{D}_{*}(M), \delta\right)$ computes the homology of $M$ with real coefficients.

We have a morphism of chain complexes

$$
C_{*}(M ; \mathbb{R}) \rightarrow \mathcal{D}_{*}(M), \quad \sigma \mapsto \hat{\sigma}, \quad \hat{\sigma}(\alpha):=\int_{\sigma} \alpha .
$$

Here $C_{*}(M ; \mathbb{R})$ denotes the space of singular chains with real coefficients. Moreover, we have a morphism of chain complexes

$$
\Omega^{n-*}\left(M ; \mathcal{O}_{M}\right) \rightarrow \mathcal{D}_{*}(M), \quad \beta \mapsto \hat{\beta}, \quad \hat{\beta}(\alpha):=(-1)^{\frac{1}{2}|\alpha|(|\alpha|+1)} \int_{M} \alpha \wedge \beta .
$$

Here $|\alpha|$ denotes the degree of $\alpha$.

Note that any zero-current represents a degree zero homology class, and $H_{0}(M ; \mathbb{R})$ identifies canonically to $\bigoplus_{\alpha} \mathbb{R}_{\alpha}$ where $\mathbb{R}_{\alpha}$ is a copy of $\mathbb{R}$ corresponding to a connected component of $M=\bigsqcup_{\alpha} M_{\alpha}$. One denotes by $\bar{\chi}(M)$ the vector $\left\{\chi\left(M_{\alpha}\right)\right\} \in \bigoplus_{\alpha} \mathbb{R}_{\alpha}$, and by $\mathcal{E}_{0}$ the affine subspace of $\mathcal{D}_{0}(M)$ consisting of zero-currents which represent $\bar{\chi}(M)$. Elements in $\mathcal{E}_{0}$ are called Euler currents.

Every vector field $X$ with isolated set of zeros $\mathcal{X}_{\tau}$ gives rise to a zero-chain $E(X)$; see Section 2.2. Via the first morphism we get a zero-current $\widehat{E}(X)$. More explicitly, $\widehat{E}(X)(h)=\sum_{x \in \mathcal{X}} \operatorname{IND}_{x}(X) h(x)$ for all functions $h \in \Omega^{0}(M ; \mathbb{R})$. By Hopf's theorem this is an Euler current.

A Riemannian metric $g$ has an Euler form $E(g) \in \Omega^{n}\left(M ; \mathcal{O}_{M}\right)$; see Section 2.1. Via the second morphism we get a zero-current $\widehat{E}(g)$ which, by Gauss-Bonnet, is an Euler current. More explicitly, $\widehat{E}(g)(h)=\int_{M} h E(g)$ for all functions $h \in \Omega^{0}(M ; \mathbb{R})$.

Let $\mathcal{Z}^{k}(M ; \mathbb{R}) \subseteq \Omega^{k}(M ; \mathbb{R})$ denote the space of closed $k$-forms on $M$ equipped with the $C^{\infty}$-topology. The continuous linear functionals on $\mathcal{Z}^{k}(M ; \mathbb{R})$ are referred 
to as $k$-currents rel. boundary and identify to $\mathcal{D}_{k}(M) / \delta\left(\mathcal{D}_{k+1}(M)\right)$. The two chain morphisms provide mappings

$$
\begin{gathered}
C_{k}(M ; \mathbb{R}) / \partial\left(C_{k+1}(M ; \mathbb{R})\right) \rightarrow \mathcal{D}_{k}(M) / \delta\left(\mathcal{D}_{k+1}(M)\right) \\
\Omega^{n-k}\left(M ; \mathcal{O}_{M}\right) / d\left(\Omega^{n-k-1}\left(M ; \mathcal{O}_{M}\right)\right) \rightarrow \mathcal{D}_{k}(M) / \delta\left(\mathcal{D}_{k+1}(M)\right) .
\end{gathered}
$$

For two vector fields $X_{1}$ and $X_{2}$ with isolated zeros, in Section 2.2 we have constructed the one-chain $\operatorname{cs}\left(X_{1}, X_{2}\right) \in C_{1}(M ; \mathbb{Z}) / \partial\left(C_{2}(M ; \mathbb{Z})\right)$ which then gives rise to $\operatorname{cs}\left(X_{1}, X_{2}\right) \in C_{1}(M ; \mathbb{R}) / \partial\left(C_{2}(M ; \mathbb{R})\right)$ and via $(25)$ we get a one-current rel. boundary which we will denote by $\widehat{\mathrm{cs}}\left(X_{1}, X_{2}\right)$. More precisely, for all closed one-forms $\omega \in \mathcal{Z}^{1}(M ; \mathbb{R})$, we have $\hat{\mathrm{cs}}\left(X_{1}, X_{2}\right)(\omega)=\int_{\mathrm{cs}\left(X_{1}, X_{2}\right)} \omega=R\left(\omega, X_{1}, X_{2}\right)$.

For two Riemannian metrics $g_{1}$ and $g_{2}$, we have the Chern-Simons form $\operatorname{cs}\left(g_{1}, g_{2}\right)$ in $\Omega^{n-1}\left(M ; \mathcal{O}_{M}\right) / d\left(\Omega^{n-2}\left(M ; \mathcal{O}_{M}\right)\right)$; see Section 2.1. Via (26) we get a one-current rel. boundary which we denote by $\widehat{\mathrm{cs}}\left(g_{1}, g_{2}\right)$. More precisely, for closed one-forms $\omega \in \mathcal{Z}^{1}(M ; \mathbb{R})$, we have $\widehat{\operatorname{cs}}\left(g_{1}, g_{2}\right)(\omega)=-\int_{M} \omega \wedge \operatorname{cs}\left(g_{1}, g_{2}\right)=-R\left(\omega, g_{1}, g_{2}\right)$.

Suppose $X$ is a vector field with isolated zeros and $g$ is a Riemannian metric. We define a one-current rel. boundary by $\widehat{\mathrm{cs}}(X, g)(\omega):=-R(\omega, X, g)$ for all closed one-forms $\omega \in \mathcal{Z}^{1}(M ; \mathbb{R})$; see Section 2.5. Moreover set $\hat{\operatorname{cs}}(g, X):=-\hat{\mathrm{cs}}(X, g)$.

Proposition 2.4 can now be reformulated.

Proposition 2.6 Let any of the symbols $x, y, z$ denote either a Riemannian metric or a vector field with isolated zeros. Then one has:

(i) $\delta \widehat{\operatorname{cs}}(x, y)=\widehat{E}(y)-\widehat{E}(x)$.

(ii) $\hat{\mathrm{cs}}(y, x)=-\hat{\mathrm{cs}}(x, y)$.

(iii) $\hat{\mathrm{cs}}(x, z)=\widehat{\mathrm{cs}}(x, y)+\widehat{\mathrm{cs}}(y, z)$.

\subsection{Smooth triangulations}

Smooth triangulations provide a remarkable source of vector fields with isolated zeros. To any smooth triangulation $\tau$ of a smooth manifold $M$ one can associate a smooth vector field $X_{\tau}$ called Euler vector field, with the following properties:

P1 The zeros of $X_{\tau}$ are all nondegenerate and are exactly the barycenters $x_{\sigma}$ of the simplices $\sigma$ of $\tau$.

P2 The piecewise differentiable function $f_{\tau}: M \rightarrow \mathbb{R}$ defined by $f_{\tau}\left(x_{\sigma}\right)=\operatorname{dim}(\sigma)$, $x_{\sigma}$ the barycenter of the simplex $\sigma$ and extended by linearity on each simplex of the barycentric subdivision of $\tau$, is a Lyapunov function for $-X_{\tau}$, ie, strictly decreasing on nonconstant trajectories of $-X_{\tau}$. 
P3 For each zero $x_{\sigma}$ the unstable set with respect to $-X_{\tau}$ agrees in some small neighborhood of $x_{\sigma}$ with the open simplex $\sigma$, consequently the zeros are hyperbolic. The Morse index of $-X_{\tau}$ at $x_{\sigma}$ equals $\operatorname{dim}(\sigma)$ and the (Hopf) index of $X_{\tau}$ at $x_{\sigma}$ equals $(-1)^{\operatorname{dim}(\sigma)}$.

The space of vector fields satisfying P1-P3 is a contractible space. The convex combination provides a contraction. It was pointed out to us that a somewhat similar vector field has been considered by Steenrod [29, Section 39.7].

To construct such a vector field we first construct a piecewise smooth (actually Lipschitz) vector field $X$ which satisfies $\mathrm{P} 1, \mathrm{P} 2$ and a stronger form of P3. Precisely the unstable sets of the rest points identify to the open simplices of the triangulation. ${ }^{4}$

We begin with a standard simplex $\Delta_{n}$ of vectors $\left(t_{0}, \ldots, t_{n}\right) \in \mathbb{R}^{n+1}$ which satisfy $0 \leq t_{i} \leq 1$ and $\sum t_{i}=1$.

(i) Let $E_{n}$ denote the Euler vector field of the corresponding affine space, $\sum t_{i}=1$, centered at the barycenter $O=(1 /(n+1), \ldots, 1 /(n+1))$ and restricted to $\Delta_{n}$.

(ii) Let $e: \Delta_{n} \rightarrow[0,1]$ denote the function which is 1 on the barycenter $O$, zero on the boundary and extended linearly on any ray through the barycenter $O$.

(iii) Let $r: \Delta_{n} \backslash\{O\} \rightarrow \partial \Delta_{n}$ denote the radial retraction to the boundary.

Set $X_{n}^{\prime}:=e \cdot E_{n}$, which is a vector field on $\Delta_{n}$.

By induction we will construct a canonical vector field $X_{n}$ on $\Delta_{n}$ which at any point $x \in \Delta_{n}$ is tangent to the open face the point belongs to and vanishes only at the barycenter of each face. We proceed as follows:

Suppose we have constructed such canonical vector fields on all $\Delta(k), k \leq n-1$. Using the canonical vector fields $X_{n-1}$ we define the vector field $X_{n}$ on the boundary $\partial \Delta_{n}$ and extend it to the vector field $X_{n}^{\prime \prime}$ by taking at each point $x \in \Delta_{n}$ the vector parallel to $X_{n}(r(x))$ multiplied by the function $(1-e)$ and at $O$ the vector zero. Clearly, such a vector field vanishes on the radii $\overline{O P}$ (P the barycenter of any face). We finally put

$$
X_{n}:=X_{n}^{\prime}+X_{n}^{\prime \prime}
$$

The vector field $X_{n}$ is continuous and piecewise differential (actually Lipschitz) and has a well-defined continuous flow.

Putting together the vector fields $X_{n}$ on all simplices we provide a piecewise differential (and Lipschitz) vector field $X$ on any simplicial complex or polyhedron and in particular

\footnotetext{
${ }^{4}$ It is not always possible to find a smooth vector field with such properties.
} 
on any smoothly triangulated manifold. The vector field $X$ has a flow and $f_{\tau}$ is a Lyapunov function for $-X$. The vector field $X$ is not necessary smooth but by a small (Lipschitz) perturbation we can approximate it by a smooth vector field which satisfies $\mathrm{P} 1-\mathrm{P} 3$. Any of the resulting vector fields is referred to as the Euler vector field of a smooth triangulation $\tau$ and denoted by $X_{\tau}$.

Remark 2.7 A similar construction can be done for smooth cell structures. Recall that a smooth cell structure on a smooth manifold $M$ is a CW-complex structure provided by cells whose characteristic maps $\chi: D^{k} \rightarrow M$ are smooth embeddings (one-to-one and of maximal rank at any point including the corners) of compact $k$-dimensional convex sets $D$, with the property that the image of the boundary is a union of open cells of lower dimension. ${ }^{5}$ We are led to a well-defined homotopy class of smooth vector fields whose rest points are the barycenters of the cells, are nondegenerate and of the same index as the dimension of the cell.

\section{Euler and co-Euler structures}

\subsection{Euler structures}

Euler structures have been introduced by Turaev [31] for manifolds with vanishing Euler-Poincaré characteristic. In the presentation below we remove the vanishing hypothesis at the expense of a base point.

Let $\left(M, x_{0}\right)$ be a base pointed closed connected manifold of dimension $n$. Let $X$ be a vector field and let $\mathcal{X}$ denote its zero set. Suppose the zeros of $X$ are isolated and recall the singular zero-chain $E(X)=\sum_{x \in \mathcal{X}} \operatorname{IND}_{X}(x) x$ from Section 2.2. An Euler chain for $X$ is a singular one-chain $c \in C_{1}(M ; \mathbb{Z})$ so that

$$
\partial c=E(X)-\chi(M) x_{0} .
$$

Since $\sum_{x \in \mathcal{X}} \operatorname{IND}_{X}(x)=\chi(M)$ every vector field with isolated zeros admits Euler chains.

Consider pairs $(X, c)$ where $X$ is a vector field with isolated zeros, and $c$ is an Euler chain for $X$. We call two such pairs $\left(X_{1}, c_{1}\right)$ and $\left(X_{2}, c_{2}\right)$ equivalent if

$$
c_{2}=c_{1}+\operatorname{cs}\left(X_{1}, X_{2}\right) \in C_{1}(M ; \mathbb{Z}) / \partial\left(C_{2}(M ; \mathbb{Z})\right) .
$$

For the definition of $\operatorname{cs}\left(X_{1}, X_{2}\right)$ see Section 2.2. We will write $\mathfrak{E u l}_{x_{0}}(M ; \mathbb{Z})$ for the set of equivalence classes as above and $[X, c] \in \mathfrak{E u l}_{x_{0}}(M ; \mathbb{Z})$ for the element represented

\footnotetext{
${ }^{5}$ More general cells can be considered but for the need of this paper (Section 4.3) this suffices.
} 
by the pair $(X, c)$. Elements of $\mathfrak{E u l}_{x_{0}}(M ; \mathbb{Z})$ are called Euler structures of $M$ based at $x_{0}$. There is an obvious $H_{1}(M ; \mathbb{Z})$ action on $\mathfrak{E u l}_{x_{0}}(M ; \mathbb{Z})$ defined by

$$
[X, c]+[\sigma]:=[X, c+\sigma],
$$

for $[\sigma] \in H_{1}(M ; \mathbb{Z})$ and $[X, c] \in \mathfrak{E u l}_{x_{0}}(M ; \mathbb{Z})$. Evidently this action is free and transitive. In this sense $\mathfrak{E u l}_{x_{0}}(M ; \mathbb{Z})$ is an affine version of $H_{1}(M ; \mathbb{Z})$.

Considering Euler chains with real coefficients one obtains, in exactly the same way, an affine version of $H_{1}(M ; \mathbb{R})$ which we will denote by $\mathfrak{E u l}_{x_{0}}(M ; \mathbb{R})$. Moreover, there is a natural map $\mathfrak{E u l}_{x_{0}}(M ; \mathbb{Z}) \rightarrow \mathfrak{E u l}_{x_{0}}(M ; \mathbb{R})$ which is affine over the homomorphism $H_{1}(M ; \mathbb{Z}) \rightarrow H_{1}(M ; \mathbb{R})$.

Remark 3.1 There is an alternative way of understanding the $H_{1}(M ; \mathbb{Z})$ action on $\mathfrak{E} \mathfrak{u l}_{x_{0}}(M ; \mathbb{Z})$. Suppose $n>2$ and represent $[\sigma] \in H_{1}(M ; \mathbb{Z})$ by a simple closed curve $\sigma$. Choose a tubular neighborhood $N$ of $\sigma\left(S^{1}\right)$ considered as vector bundle $N \rightarrow S^{1}$. Choose a fiber metric and a linear connection on $N$. Choose a representative of $[X, c] \in \mathfrak{E u l}_{x_{0}}(M ; \mathbb{Z})$ such that $\left.X\right|_{N}=\frac{\partial}{\partial \theta}$, the horizontal lift of the canonical vector field on $S^{1}$. Choose a function $\lambda:[0, \infty) \rightarrow[-1,1]$, which satisfies $\lambda(r)=-1$ for $r \leq \frac{1}{3}$ and $\lambda(r)=1$ for $r \geq \frac{2}{3}$. Finally, choose a function $\mu:[0, \infty) \rightarrow \mathbb{R}$ satisfying $\mu(r)=r$ for $r \leq \frac{1}{3}, \mu(r)=0$ for $r \geq \frac{2}{3}$ and $\mu(r)>0$ for all $r \in\left(\frac{1}{3}, \frac{2}{3}\right)$. Now construct a new smooth vector field $\tilde{X}$ on $M$ by setting

$$
\tilde{X}:= \begin{cases}X & \text { on } M \backslash N \\ \lambda(r) \frac{\partial}{\partial \theta}+\mu(r) \frac{\partial}{\partial r} & \text { on } N\end{cases}
$$

where $r: N \rightarrow[0, \infty)$ denotes the radius function determined by the fiber metric on $N$ and $-r \frac{\partial}{\partial r}$ is the Euler vector field of $N$. This construction is known as Reeb surgery [25]. If the zeros of $X$ are all nondegenerate the homotopy $X_{t}:=(1-t) X+t \tilde{X}$ is a nondegenerate homotopy from $X_{0}=X$ to $X_{1}=\tilde{X}$ from which one easily deduces that $[\tilde{X}, c]=[X, c]+[\sigma]$. Particularly, all the choices that entered the Reeb surgery do not affect the out-coming Euler structure $[\tilde{X}, c]$.

Let us consider a change of base point. Let $x_{0}, x_{1} \in M$ and choose a path $\sigma$ from $x_{0}$ to $x_{1}$. Define

$$
\mathfrak{E u l}_{x_{0}}(M ; \mathbb{Z}) \rightarrow \mathfrak{E u l}_{x_{1}}(M ; \mathbb{Z}), \quad[X, c] \mapsto[X, c-\chi(M) \sigma] .
$$

This is an $H_{1}(M ; \mathbb{Z})$ equivariant bijection but depends on the homology class of $\sigma$ rel. $\left\{x_{0}, x_{1}\right\}$. However, if $\chi(M)=0$, then $\mathfrak{E u l}_{x_{0}}(M ; \mathbb{Z})$ does not depend on the base point [31], and we will write $\mathfrak{E u l}(M ; \mathbb{Z})$ in this case. 
Remark 3.2 The assignment $v([X, c]):=\left[-X,(-1)^{n} c\right]$ defines an involution $v$ on $\mathfrak{E u l}_{x_{0}}(M ; \mathbb{R})$; see (17) and (18). It is affine over the homomorphism $(-1)^{n}$ from $H_{1}(M ; \mathbb{R})$ to $H_{1}(M ; \mathbb{R})$. Hence $v=$ id for even $n$. If $n$ is odd then $v$ has a unique fixed point which we denote by $\mathfrak{e}_{\text {can }} \in \mathfrak{E} \mathfrak{u l}(M ; \mathbb{R})$ and referred to as the canonical Euler structure. It can be represented as $\mathfrak{e}_{\mathrm{can}}=\left[X, \frac{1}{2} \operatorname{cs}(-X, X)\right]$ and this does not depend on $X$. Hence we obtain a canonical identification $H_{1}(M ; \mathbb{R})=\mathfrak{E u l}(M ; \mathbb{R})$, $\sigma \mapsto \mathfrak{e}_{\text {can }}+\sigma$, provided $n$ is odd. This involution makes sense on $\mathfrak{E u l}_{x_{0}}(M ; \mathbb{Z})$ too, but neither must it be trivial for even $n$, nor must it possess a (unique) fixed point if $n$ is odd. Finally, note that the natural mapping $\mathfrak{E u l}_{x_{0}}(M ; \mathbb{Z}) \rightarrow \mathfrak{E u l}_{x_{0}}(M ; \mathbb{R})$ obviously intertwines the involutions on $\mathfrak{E u l}_{x_{0}}(M ; \mathbb{Z})$ and $\mathfrak{E u l}_{x_{0}}(M ; \mathbb{R})$.

Lemma 3.3 Suppose $X$ is a smooth vector field with isolated zeros, $\mathfrak{e} \in \mathfrak{E} u l_{x_{0}}(M ; \mathbb{Z})$ and assume $X$ has at least one zero with Hopf index \pm 1 . Then one can choose a collection of smooth paths $\pi_{x}:[0,1] \rightarrow M$ with $\pi_{x}(0)=x_{0}$ and $\pi_{x}(1)=x, x \in \mathcal{X}$ so that for $c:=\sum_{x \in \mathcal{X}} \operatorname{IND}_{X}(x) \pi_{x}$ one has $\mathfrak{e}=[X, c]$. Moreover, if $\operatorname{dim} M>2$ one can choose these paths to be smoothly embedded and so that they only intersect at $x_{0}$.

Proof Let $\pi_{x}^{\prime}:[0,1] \rightarrow M$ be any collection of smooth paths with $\pi_{x}^{\prime}(0)=x_{0}$ and $\pi_{x}^{\prime}(1)=x, x \in \mathcal{X}$. Then $c^{\prime}:=\sum_{x \in \mathcal{X}} \operatorname{IND}_{X}(x) \pi_{x}^{\prime}$ is an Euler chain for $X$, and we get an Euler structure $\mathfrak{e}^{\prime}:=\left[X, c^{\prime}\right] \in \mathfrak{E u l}_{x_{0}}(M ; \mathbb{Z})$. Since the $H_{1}(M ; \mathbb{Z})$ action on $\mathfrak{E u l}_{x_{0}}(M ; \mathbb{Z})$ is transitive and the Huréwicz homomorphism $\pi_{1}\left(M, x_{0}\right) \rightarrow H_{1}(M ; \mathbb{Z})$ is onto, we find a closed smooth path $\sigma:[0,1] \rightarrow M, \sigma(0)=\sigma(1)=x_{0}$ satisfying $\mathfrak{e}=\mathfrak{e}^{\prime}+[\sigma]$. Choose a zero $y \in \mathcal{X}$ with $\operatorname{IND}_{X}(y)= \pm 1$. Define $\pi_{y}$ as the concatenation $\sigma^{\operatorname{IND}_{X}(y)} * \pi_{y}^{\prime}$, and set $\pi_{x}:=\pi_{x}^{\prime}$ for $y \neq x \in \mathcal{X}$. Then $c:=\sum_{x \in \mathcal{X}} \operatorname{IND}_{X}(x) \pi_{x}$ is an Euler chain for $X$ and $[X, c]=\mathfrak{e}^{\prime}+\operatorname{IND}_{X}(y)^{2}[\sigma]=\mathfrak{e}$.

If $\operatorname{dim}(M)>2$ it is possible to approximate the paths $\pi_{x}$ by smoothly embedded paths $\tilde{\pi}_{x}$ which intersect only at $x_{0}$, have the same endpoints, and such that the one-chain $\tilde{\pi}_{x}-\pi_{x} \in C_{1}(M ; \mathbb{Z})$ is a boundary. Then $\tilde{c}:=\sum_{x \in \mathcal{X}} \operatorname{IND}_{X}(x) \tilde{\pi}_{x}$ is an Euler chain for $X$ and $[X, \widetilde{c}]=[X, c]=\mathfrak{e}$.

Let $\mathfrak{X}\left(M, x_{0}\right)$ denote the space of vector fields which vanish at $x_{0}$ and are nonzero elsewhere. We equip this space with the $C^{\infty}$-topology. Let $\pi_{0}\left(\mathfrak{X}\left(M, x_{0}\right)\right)$ denote the set of homotopy classes of such vector fields. If $X \in \mathfrak{X}\left(M, x_{0}\right)$ we will write $[X]$ for the corresponding class in $\pi_{0}\left(\mathfrak{X}\left(M, x_{0}\right)\right)$. The following proposition is due to Turaev in the case $\chi(M)=0$.

Proposition 3.4 Suppose $n>2$. Then there exists a natural bijection

$$
\pi_{0}\left(\mathfrak{X}\left(M, x_{0}\right)\right)=\mathfrak{E u l}_{x_{0}}(M ; \mathbb{Z}), \quad[X] \mapsto[X, 0] .
$$


Proof To see that (28) is well-defined note that for $\left[X_{1}\right]=\left[X_{2}\right] \in \pi_{0}\left(\mathfrak{X}\left(M, x_{0}\right)\right)$ we find a representative of $\operatorname{cs}\left(X_{1}, X_{2}\right)$ which is supported in a contractible open neighborhood $U$ of $x_{0}$. Since $H_{1}(U ; \mathbb{Z})=0$ this representative is a boundary, hence $\left[X_{1}, 0\right]=\left[X_{2}, 0\right] \in \mathfrak{E u l}_{x_{0}}(M ; \mathbb{Z})$.

To see that (28) is onto let $\mathfrak{e} \in \mathfrak{E u l}_{x_{0}}(M ; \mathbb{Z})$ be an Euler structure. Choose a vector field $X$ on $M$ with isolated zero set $\mathcal{X}$ such that it has at least one zero with Hopf index \pm 1 . In view of Lemma 3.3 there exists an Euler chain $c$ for $X$ with $[X, c]=\mathfrak{e}$ and such that $c$ is supported in a compact contractible set containing $x_{0}$. Hence we find a smoothly embedded open disk $D \subseteq M$ so that $x_{0} \in D, \mathcal{X} \subseteq D$ and so that $c$ is supported in $D$. Choose a vector field $X^{\prime}$ which coincides with $X$ on a neighborhood of $M \backslash D$ and vanishes just at $x_{0}$. Then there exists a representative of $\operatorname{cs}\left(X, X^{\prime}\right)$ which is supported in $D$. Since $H_{1}(D ; \mathbb{Z})=0$ the difference of this representative and $c$ must be a boundary and we obtain $\left[X^{\prime}, 0\right]=[X, c]=\mathfrak{e}$. Therefore (28) is onto.

To prove injectivity of (28) let $X_{1}, X_{2} \in \mathfrak{X}\left(M, x_{0}\right)$ and suppose $\left[X_{1}, 0\right]=\left[X_{2}, 0\right] \in$ $\mathfrak{E u l}_{x_{0}}(M ; \mathbb{Z})$, ie, $\operatorname{cs}\left(X_{1}, X_{2}\right)=0 \in H_{1}(M ; \mathbb{Z})$. Let $\bar{D} \subseteq M$ denote an embedded closed disk (of full dimension) centered at $x_{0}$, and let $D$ denote its interior. Consider the vector bundle $p^{*} T M \rightarrow I \times M$ and consider the two vector fields as a nowhere vanishing section of $p^{*} T M$ defined over the set $\partial I \times \dot{M}$, where $\dot{M}:=M \backslash D$. We would like to extend it to a nowhere vanishing section over $I \times \dot{M}$. The first obstruction we meet is an element in

$$
\begin{aligned}
H^{n}\left(I \times \dot{M}, \partial I \times \dot{M} ;\left\{\pi_{n-1}\right\}\right) & =H_{1}(I \times \dot{M}, I \times \partial D ; \mathbb{Z}) \\
& =H_{1}(M, \bar{D} ; \mathbb{Z}) \\
& =H_{1}(M ; \mathbb{Z})
\end{aligned}
$$

which corresponds to $\operatorname{cs}\left(X_{1}, X_{2}\right)=0$. Here $\left\{\pi_{n-1}\right\}$ denotes the system of local coefficients determined by the sphere bundle of $p^{*} T M$ with $\pi_{n-1}=\pi_{n-1}\left(S^{n-1}\right)$. Since this obstruction vanishes by hypothesis the next obstruction is defined and is an element in

$$
\begin{aligned}
H^{n+1}\left(I \times \dot{M}, \partial I \times \dot{M} ;\left\{\pi_{n}\right\}\right) & =H_{0}\left(I \times \dot{M}, I \times \partial D ; \pi_{n}\left(S^{n-1}\right)\right) \\
& =H_{0}\left(M, \bar{D} ; \pi_{n}\left(S^{n-1}\right)\right) \\
& =0 .
\end{aligned}
$$

So obstruction theory as in Whitehead [34] tells us that we find a nowhere vanishing section of $p^{*} T M$ defined over $I \times \dot{M}$, which restricts to $X_{i}$ on $\{i\} \times \dot{M}, i=1,2$. Such a section can easily be extended to a globally defined section of $p^{*} T M \rightarrow I \times M$, 
which restricts to $X_{i}$ on $\{i\} \times M, i=1,2$, and whose zero set is precisely $I \times\left\{x_{0}\right\}{ }^{6}$ The resulting globally defined section can be considered as homotopy from $X_{1}$ to $X_{2}$ showing $\left[X_{1}\right]=\left[X_{2}\right] \in \pi_{0}\left(\mathfrak{X}\left(X, x_{0}\right)\right)$. Hence (28) is injective.

Remark 3.5 Provided $n>2$, Reeb surgery defines an $H_{1}(M ; \mathbb{Z})$ action on the set $\pi_{0}\left(\mathfrak{X}\left(M, x_{0}\right)\right)$ which via (28) corresponds to the $H_{1}(M ; \mathbb{Z})$ action on $\mathfrak{E u l}_{x_{0}}(M ; \mathbb{Z})$; cf Remark 3.1.

Let $\mathfrak{X}_{0}(M)$ denote the space of nowhere vanishing vector fields on $M$ equipped with the $C^{\infty}$-topology. Let $\pi_{0}\left(\mathfrak{X}_{0}(M)\right)$ denote the set of its connected components. For the next statement see also Turaev [31].

Proposition 3.6 Suppose $\chi(M)=0$ and $n>2$. Then we have a surjection

$$
\pi_{0}\left(\mathfrak{X}_{0}(M)\right) \rightarrow \mathfrak{E u l}_{x_{0}}(M ; \mathbb{Z}), \quad[X] \mapsto[X, 0] .
$$

Proof The assignment (29) is well-defined since for $\left[X_{1}\right]=\left[X_{2}\right] \in \pi_{0}\left(\mathfrak{X}_{0}(M)\right)$ the Chern-Simons class $\operatorname{cs}\left(X_{1}, X_{2}\right)$ can be represented by 0 .

To prove surjectivity let $\mathfrak{e} \in \mathfrak{E u l}_{x_{0}}(M ; \mathbb{Z})$ be an Euler structure. As explained in the proof of Proposition 3.4 we find a vector field $X$ and an Euler chain $c$ for $X$ with $\mathfrak{e}=[X, c]$ and such that there exists a smoothly embedded open disk $D$ with $x_{0} \in D, \mathcal{X} \subseteq D$ and so that $c$ is supported in $D$. Wlog we may assume that $\bar{D}$ is a smoothly embedded closed disk in $M$. Since $\chi(M)=0$ the degree of the map $X: \partial \bar{D} \rightarrow T \bar{D} \backslash \bar{D}$ vanishes. Hence we find a nowhere vanishing $X^{\prime}$ which coincides with $X$ on a neighborhood of $M \backslash D$. Hence $\operatorname{cs}\left(X, X^{\prime}\right)$ admits a representative supported in $D$. Since $H_{1}(D ; \mathbb{Z})=0$ the difference of this representative and $c$ must be a boundary and we obtain $\left[X^{\prime}, 0\right]=[X, c]=\mathfrak{e}$. Therefore (29) is onto.

\subsection{Co-Euler structures}

We will now describe another approach to Euler structures which is in some sense Poincaré dual to the one in Section 3.1. We still consider a closed connected base pointed manifold $\left(M, x_{0}\right)$ of dimension $n$. Consider pairs $(g, \alpha)$ where $g$ is a Riemannian metric on $M$ and $\alpha \in \Omega^{n-1}\left(M \backslash x_{0} ; \mathcal{O}_{M}\right)$ with $d \alpha$ equal to the Euler class $E(g) \in$ $\Omega^{n}\left(M ; \mathcal{O}_{M}\right)$ of $g$; see Section 2.1. Since $H^{n}\left(M \backslash x_{0} ; \mathcal{O}_{M}\right)=0$ every Riemannian metric admits such $\alpha$. We call two pairs $\left(g_{1}, \alpha_{1}\right)$ and $\left(g_{2}, \alpha_{2}\right)$ equivalent if

$$
\operatorname{cs}\left(g_{1}, g_{2}\right)=\alpha_{2}-\alpha_{1} \in \Omega^{n-1}\left(M \backslash x_{0} ; \mathcal{O}_{M}\right) / d \Omega^{n-2}\left(M \backslash x_{0} ; \mathcal{O}_{M}\right) .
$$

\footnotetext{
${ }^{6}$ For example trivialize the bundle $p^{*} T M$ over $I \times \bar{D}$ and extend the section on $\partial(I \times \bar{D})$ to $I \times \bar{D}$ by scaling radially centered at $\left(\frac{1}{2}, x_{0}\right) \in I \times \bar{D}$.
} 
Write $\mathfrak{E u r}_{x_{0}}^{*}(M ; \mathbb{R})$ for the set of equivalence classes and $[g, \alpha]$ for the equivalence class represented by the pair $(g, \alpha)$. Elements of $\mathfrak{E u l}_{x_{0}}^{*}(M ; \mathbb{R})$ are called co-Euler structures based at $x_{0}$. There is a natural $H^{n-1}\left(M ; \mathcal{O}_{M}\right)$ action on $\mathfrak{E u l}_{x_{0}}^{*}(M ; \mathbb{R})$ given for $[\beta] \in H^{n-1}\left(M ; \mathcal{O}_{M}\right)$ by

$$
[g, \alpha]+[\beta]:=[g, \alpha-\beta] .
$$

Since $H^{n-1}\left(M ; \mathcal{O}_{M}\right)=H^{n-1}\left(M \backslash x_{0} ; \mathcal{O}_{M}\right)$ this action is obviously free and transitive. In this sense $\mathfrak{E u l}_{x_{0}}^{*}(M ; \mathbb{R})$ is an affine version of $H^{n-1}\left(M ; \mathcal{O}_{M}\right)$.

Remark 3.7 Given a Riemannian metric $g$ and a class $\mathfrak{e}^{*} \in \mathfrak{E u}_{x_{0}}^{*}(M ; \mathbb{R})$ one can find $\alpha \in \Omega^{n-1}\left(M \backslash x_{0} ; \mathcal{O}_{M}\right)$ with $d \alpha=E(g)$ so that $\mathfrak{e}^{*}=[g, \alpha]$. Moreover, if $\chi(M)=0$ one can choose $\alpha \in \Omega^{n-1}\left(M ; \mathcal{O}_{M}\right)$. Hence in the case of vanishing Euler characteristic the set of co-Euler structures is defined without reference to a base point and we will denote it by $\mathfrak{E u}^{*}(M ; \mathbb{R})$.

Remark 3.8 Suppose $n$ is odd. Then $\chi(M)=0, E(g)=0$ and $\operatorname{cs}\left(g_{1}, g_{2}\right)=0$ for all Riemannian metrics $g, g_{1}$ and $g_{2}$; see (6) and (7). Hence $[g, 0]$ represents a co-Euler structure independent of the Riemannian metric $g$. We refer to this co-Euler structure as the canonical co-Euler structure and will denote it as $\mathfrak{e}_{\text {can }}^{*} \in \mathfrak{E u}^{*}(M ; \mathbb{R})$. It provides a canonical identification $H^{n-1}\left(M ; \mathcal{O}_{M}\right)=\mathfrak{E u l}^{*}(M ; \mathbb{R}), \beta \mapsto \mathfrak{e}_{\text {can }}^{*}+\beta$. This too can be understood in terms of the involution $[g, \alpha] \mapsto\left[g,(-1)^{n} \alpha\right]$ on $\mathfrak{E u l}_{x_{0}}^{*}(M ; \mathbb{R})$; cf Remark 3.2.

Suppose $[X, c] \in \mathfrak{E u l}_{x_{0}}(M ; \mathbb{R}),[g, \alpha] \in \mathfrak{E u r}_{x_{0}}^{*}(M ; \mathbb{R})$ and let $\omega \in \Omega^{1}(M ; \mathbb{R})$ be a closed one-form. Then the real number

$$
R(\omega, X, g)-S(\omega, \alpha)-\int_{c} \omega
$$

does only depend on the Euler structure $[X, c] \in \mathfrak{E u l}_{x_{0}}(M ; \mathbb{R})$, the co-Euler structure $[g, \alpha] \in \mathfrak{E u}_{x_{0}}^{*}(M ; \mathbb{R})$ and the cohomology class $[\omega] \in H^{1}(M ; \mathbb{R})$. Indeed, in view of Lemma 2.3(i), Proposition 2.4(i) and (iv) as well as $d \alpha=E(g), \partial c=E(X)-\chi(M) x_{0}$ and $\operatorname{IND}_{\alpha}\left(x_{0}\right)=\chi(M) x_{0}$ the quantity (30) remains unchanged when $\omega$ is replaced by $\omega+d h$. Proposition 2.4(x) and (23) show that it does not depend on the representative of the Euler structure $[X, c]$. In view of Proposition 2.4(ix) and (22) it does not depend on the representative of the co-Euler structure either. Thus (30) defines a coupling

$$
\mathbb{T}: \mathfrak{E u l}_{x_{0}}(M ; \mathbb{R}) \times \mathfrak{E} \mathfrak{l}_{x_{0}}^{*}(M ; \mathbb{R}) \rightarrow H_{1}(M ; \mathbb{R}) .
$$

From the very definition we have

$$
\langle[\omega], \mathbb{T}([X, c],[g, \alpha])\rangle=\int_{M} \omega \wedge\left(X^{*} \Psi(g)-\alpha\right)-\int_{c} \omega,
$$


where $\omega$ is any representative of $[\omega]$ which vanishes locally around the zeros of $X$ and vanishes locally around the base point $x_{0}$. Moreover, we have

$$
\mathbb{T}\left(\mathfrak{e}+\sigma, \mathfrak{e}^{*}+\beta\right)=\mathbb{T}\left(\mathfrak{e}, \mathfrak{e}^{*}\right)-\sigma+\mathrm{PD}^{-1}(\beta)
$$

for all $\mathfrak{e} \in \mathfrak{E u l}_{x_{0}}(M ; \mathbb{R}), \mathfrak{e}^{*} \in \mathfrak{E u l}_{x_{0}}^{*}(M ; \mathbb{R}), \sigma \in H_{1}(M ; \mathbb{R})$ and $\beta \in H^{n-1}\left(M ; \mathcal{O}_{M}\right)$. Here PD: $H_{1}(M ; \mathbb{R}) \rightarrow H^{n-1}\left(M ; \mathcal{O}_{M}\right)$ denotes the Poincaré duality isomorphism.

We have the following affine version of Poincare duality:

Proposition 3.9 There is a natural isomorphism

$$
P: \mathfrak{E u l}_{x_{0}}(M ; \mathbb{R}) \rightarrow \mathfrak{E u l}_{x_{0}}^{*}(M ; \mathbb{R})
$$

affine over the Poincaré duality isomorphism PD: $H_{1}(M ; \mathbb{R}) \rightarrow H^{n-1}\left(M ; \mathcal{O}_{M}\right)$. More precisely, for $\sigma \in H_{1}(M ; \mathbb{R})$ and $\mathfrak{e} \in \mathfrak{E u l}_{x_{0}}(M ; \mathbb{R})$ we have

$$
P(\mathfrak{e}+\sigma)=P(\mathfrak{e})+\operatorname{PD}(\sigma) .
$$

This affine isomorphism intertwines the involution on $\mathfrak{E u l}_{x_{0}}(M ; \mathbb{R})$ with the involution on $\mathfrak{E u r}_{x_{0}}^{*}(M ; \mathbb{R})$, that is, $P(v(\mathfrak{e}))=v(P(\mathfrak{e}))$ for all $\mathfrak{e} \in \mathfrak{E u l}_{x_{0}}(M ; \mathbb{R})$. Moreover, $\mathbb{T}\left(\mathfrak{e}, \mathfrak{e}^{*}\right)=P^{-1}\left(\mathfrak{e}^{*}\right)-\mathfrak{e}$.

Proof In view of (32) we may define $P$ by $\mathbb{T}(\mathfrak{e}, P(\mathfrak{e}))=0$. The equivariance property and the last equation follow at once. The bijectivity of $P$ follows from the bijectivity of PD. The statement $P(v(\mathfrak{e}))=v(P(\mathfrak{e}))$ is a consequence of $(10)$; see Remark 3.2 and Remark 3.8.

Let us collect the observations from the last two sections.

Theorem 3.10 Let $\left(M, x_{0}\right)$ be a closed connected base-pointed manifold.

(i) Let $\pi_{0}\left(\mathfrak{X}\left(M, x_{0}\right)\right)$ denote the set of connected components of the space of vector fields which just vanish in $x_{0}$, equipped with the $C^{\infty}$-topology. If $\operatorname{dim} M>2$ then we have a bijection

$$
\pi_{0}\left(\mathfrak{X}\left(M, x_{0}\right)\right) \rightarrow \mathfrak{E u l}_{x_{0}}(M ; \mathbb{Z}), \quad[X] \mapsto[X, 0] .
$$

(ii) Let $\pi_{0}\left(\mathfrak{X}_{0}(M)\right)$ denote the set of connected components of the space of nowhere vanishing vector fields, equipped with the $C^{\infty}$-topology. If $\chi(M)=0$ and $\operatorname{dim} M>2$ we have a surjection

$$
\pi_{0}\left(\mathfrak{X}_{0}(M)\right) \rightarrow \mathfrak{E u l}_{x_{0}}(M ; \mathbb{Z}), \quad[X] \mapsto[X, 0] .
$$


(iii) There exists a natural isomorphism

$$
P: \mathfrak{E u l}_{x_{0}}(M ; \mathbb{R}) \rightarrow \mathfrak{E u l}_{x_{0}}^{*}(M ; \mathbb{R})
$$

affine over the Poincaré duality PD: $H_{1}(M ; \mathbb{R}) \rightarrow H^{n-1}\left(M ; \mathcal{O}_{M}\right)$, that is, $P(\mathfrak{e}+\sigma)=P(\mathfrak{e})+\operatorname{PD}(\sigma)$, for all $\sigma \in H_{1}(M ; \mathbb{R})$.

(iv) The assignment $\mathbb{T}$ : $\mathfrak{E u l}_{x_{0}}(M ; \mathbb{R}) \times \mathfrak{E} u l_{x_{0}}^{*}(M ; \mathbb{R}) \rightarrow H_{1}(M ; \mathbb{R})$ which is given by $\mathbb{T}\left(\mathfrak{e}, \mathfrak{e}^{*}\right)=P^{-1}\left(\mathfrak{e}^{*}\right)-\mathfrak{e}$ satisfies

$$
\left\langle[\omega], \mathbb{T}\left(\mathfrak{e}, \mathfrak{e}^{*}\right)\right\rangle=\int_{M} \omega \wedge\left(X^{*} \Psi(g)-\alpha\right)-\int_{c} \omega
$$

where $\mathfrak{e}=[X, c], \mathfrak{e}^{*}=[g, \alpha]$ and $\omega \in \Omega^{1}(M ; \mathbb{R})$ is any representative of the class $[\omega] \in H^{1}(M ; \mathbb{R})$ which vanishes locally around $x_{0}$ and the zeros of $X$.

We continue to denote by $P$ resp. PD the composition of $P$ resp. PD with the canonical mapping $\mathfrak{E} u l_{x_{0}}(M ; \mathbb{Z}) \rightarrow \mathfrak{E u l}_{x_{0}}(M ; \mathbb{R})$ resp. the homomorphism $H_{1}(M ; \mathbb{Z}) \rightarrow$ $H_{1}(M ; \mathbb{R})$. The image $P\left(\mathfrak{E u l}_{x_{0}}(M ; \mathbb{Z})\right) \subseteq \mathfrak{E u l}_{x_{0}}(M ; \mathbb{R})$ is referred to as the lattice of integral co-Euler structures. If two Euler structures $\mathfrak{e}_{1}, \mathfrak{e}_{2} \in \mathfrak{E u l}_{x_{0}}(M ; \mathbb{Z})$ satisfy $P\left(\mathfrak{e}_{1}\right)=P\left(\mathfrak{e}_{2}\right)$ then $\mathfrak{e}_{2}-\mathfrak{e}_{1} \in H_{1}(M ; \mathbb{Z})$ is a torsion element.

\subsection{Milnor-Turaev torsion}

Let $M$ be a closed connected manifold of dimension $n$. Fix a base point $x_{0} \in M$ and let $\Gamma=\pi_{1}\left(M, x_{0}\right)$ denote the fundamental group. Let $\mathbb{K}$ be a field of characteristic zero and let $V$ be a finite-dimensional vector space over $\mathbb{K}$. Let $\rho: \Gamma \rightarrow \operatorname{GL}(V)$ be a representation. Consider the graded $\mathbb{K}$-vector space $H^{*}(M ; \rho)$, its determinant line $\operatorname{det} H^{*}(M ; \rho)$, and the one-dimensional $\mathbb{K}$-vector space ${ }^{7}$

$$
\operatorname{Det}_{x_{0}}(M ; \rho):=\operatorname{det} H^{*}(M ; \rho) \otimes(\operatorname{det} V)^{-\chi(M)} .
$$

Remark 3.11 If $\chi(M)=0$ then $\operatorname{Det}_{x_{0}}(M ; \rho)=\operatorname{det} H^{*}(M ; \rho)$. If $H^{*}(M ; \rho)=0$ then $\operatorname{Det}_{x_{0}}(M ; \rho)$ is canonically isomorphic to $\mathbb{K}$.

Suppose we have a smooth triangulation $\tau$ of $M$. It gives rise to a cochain complex of $\mathbb{K}$-vector spaces $C_{\tau}^{*}(M ; \rho)$ which computes the cohomology $H^{*}(M ; \rho)$. By standard linear algebra we get a canonical isomorphism of $\mathbb{K}$-vector spaces

$$
\varphi_{\tau}^{\rho}: \operatorname{det} C_{\tau}^{*}(M ; \rho) \rightarrow \operatorname{det} H\left(C_{\tau}^{*}(M ; \rho)\right)=\operatorname{det} H^{*}(M ; \rho) .
$$

\footnotetext{
${ }^{7}$ For the definition of (graded) determinant lines see the footnote in the introduction.
} 
This isomorphism is described in detail by Farber and Turaev [15, section 2, formula (2.2)] where a sign correction ${ }^{8}(-1)^{N(C)}$ of previously used definitions is added; see also (62) in the appendix of this paper.

Recall the Euler vector field $X_{\tau}$ of $\tau$ from Section 2.7. Its zero set $\mathcal{X}_{\tau}$ coincides with the set of barycenters of $\tau$. For a cell $\sigma$ of $\tau$ we let $x_{\sigma} \in \mathcal{X}_{\tau}$ denote its barycenter. We have $\operatorname{IND}_{X_{\tau}}\left(x_{\sigma}\right)=(-1)^{\operatorname{dim} \sigma}$ for every cell $\sigma$ of $\tau$. Suppose we have given an Euler structure $\mathfrak{e} \in \mathfrak{E u l}_{x_{0}}(M ; \mathbb{Z})$. By Lemma 3.3, see also Turaev [31], one can choose a collection of paths $\pi_{x}$ from $x_{0}$ to $x \in \mathcal{X}_{\tau}$ so that with $c:=\sum_{x \in \mathcal{X}_{\tau}} \operatorname{IND}_{X_{\tau}}(x) \pi_{x}$ we have $\mathfrak{e}=\left[X_{\tau}, c\right]$.

Let $v$ be a nonzero element in $\operatorname{det} V .{ }^{9}$ Using parallel transport along $\pi_{x}$ we get a nonzero element in $\operatorname{det} C_{\tau}^{*}(M ; \rho)$ with a sign ambiguity. If the set of barycenters $\mathcal{X}_{\tau}$ were ordered we would get a well-defined nonzero element in $\operatorname{det} C_{\tau}^{*}(M ; \rho)$.

Let $\mathfrak{o}$ be a cohomology orientation of $M$, ie, an orientation of det $H^{*}(M ; \mathbb{R})$. We say an ordering of the zeros is compatible with $\mathfrak{o}$ if the nonzero element in $\operatorname{det} C_{\tau}^{*}(M ; \mathbb{R})$ provided by this ordered base is compatible with the orientation $\mathfrak{o}$ via the canonical isomorphism

$$
\varphi_{\tau}^{\mathbb{R}}: \operatorname{det} C_{\tau}^{*}(M ; \mathbb{R}) \rightarrow \operatorname{det} H\left(C_{\tau}^{*}(M ; \mathbb{R})\right)=\operatorname{det} H^{*}(M ; \mathbb{R}) .
$$

So given a collection of paths $\pi_{x}$ with $\mathfrak{e}=\left[X_{\tau}, c\right], c=\sum_{x \in X_{\tau}} \operatorname{IND}_{X_{\tau}}(x) \pi_{x}$, an ordering of $\mathcal{X}_{\tau}$ compatible with $\mathfrak{o}$, and a nonzero element $v \in \operatorname{det} V$ one obtains (using Milnor's construction modified by Turaev) a nonzero element in $\operatorname{det} C_{\tau}^{*}(M ; \rho)$ corresponding to a nonzero element in $\operatorname{det} H^{*}(M ; \rho)$ via (34). We thus get a mapping

$$
\operatorname{det} V \backslash 0 \rightarrow \operatorname{det} H^{*}(M ; \rho) \backslash 0 .
$$

This mapping is obviously homogeneous of degree $\chi(M)$. A straight forward calculation shows that it is independent on the choice of $\pi_{x}$ or the ordering on $\mathcal{X}_{\tau}$ provided they define the same $\mathfrak{e}$ and $\mathfrak{o}$. As a matter of fact this mapping does not depend on $\tau$ either but only on the Euler structure $\mathfrak{e}$ and the cohomology orientation $\mathfrak{o}$. In fact it depends on $\mathfrak{o}$ only when $\operatorname{dim} V$ is odd; $\operatorname{cf}(36)$ below. This is a nontrivial fact, and its proof is contained in Milnor [24] and Turaev [30] for the acyclic case, and implicit in the existing literature [15], but see also Corollary A.3 in the appendix.

Definition 3.12 (Milnor-Turaev torsion) The element in $\operatorname{Det}_{x_{0}}(M ; \rho)$ corresponding to the homogeneous mapping (35) is referred to as the Milnor-Turaev torsion and will be denoted by $\tau_{\text {comb }}^{\rho, \mathfrak{e}, \mathfrak{o}}$; see (33).

\footnotetext{
${ }^{8}$ first introduced in [30]

${ }^{9}$ Note that a frame (basis) in $V$ determines such an element. Note that if instead of $v$ we pick up a frame $\epsilon$ of $V$, we will obtain a base in $C_{\tau}^{*}(M ; \rho)$.
} 
We obviously have

$$
\tau_{\mathrm{comb}}^{\rho, \mathfrak{e}+\sigma, \mathfrak{o}}=\tau_{\mathrm{comb}}^{\rho, \mathfrak{e}, \mathfrak{o}} \cdot[\operatorname{det} \circ \rho](\sigma)^{-1}, \quad \text { for all } \sigma \in H_{1}(M ; \mathbb{Z}) .
$$

See Remark 2.1 for the sign. Here [det $\circ \rho]: H_{1}(M ; \mathbb{Z}) \rightarrow \mathbb{K}^{*}$ denotes the homomorphism induced from the homomorphism det $\circ \rho: \Gamma \rightarrow \mathbb{K}^{*}$. In particular, if $\rho$ is an unimodular representation then $\tau_{\text {comb }}^{\rho, \mathfrak{e}, \mathfrak{o}}$ is independent of $\mathfrak{e}$. Moreover

$$
\tau_{\mathrm{comb}}^{\rho, \mathfrak{e},-\mathfrak{o}}=(-1)^{\operatorname{dim} V} \tau_{\mathrm{comb}}^{\rho, \mathfrak{e}, \mathfrak{o}} .
$$

Remark 3.13 Note that when $H^{*}(M ; \rho)=0$ then, in view of the canonical isomorphism $\operatorname{Det}_{x_{0}}(M ; \rho)=\mathbb{K}$ of Remark 3.11, the Milnor-Turaev torsion is an element in $\mathbb{K}^{*}$. To stay with traditional notation we will denote the inverse of this number by $\mathcal{T}_{\text {comb }}^{\mathfrak{e}, \mathfrak{o}}(\rho):=\left(\tau_{\text {comb }}^{\rho, \mathfrak{e}, \mathfrak{o}}\right)^{-1}$, and later consider it as a function on the space of acyclic representations.

\subsection{Modified Ray-Singer metric}

Let us briefly recall the definition of the Ray-Singer torsion [28]. Suppose $\mathbb{F}=(F, \nabla)$ is a real or complex vector bundle $F$ equipped with a flat connection $\nabla$. Let $g$ be a Riemannian metric on $M$ and let $\mu$ be a Hermitian metric on $F$. The Ray-Singer torsion $T_{\mathrm{an}}(\nabla, g, \mu)$ is the positive real number given by

$$
\log T_{\mathrm{an}}(\nabla, g, \mu):=\frac{1}{2} \sum_{k}(-1)^{k+1} k \log \operatorname{det}^{\prime} \Delta^{k},
$$

where $\Delta^{k}$ is the Laplacian acting in degree $k$ of the elliptic complex $\left(\Omega^{*}(M ; F), d_{\nabla}\right)$ equipped with the scalar product induced from the Riemannian metric $g$ and the Hermitian metric $\mu$, and $\operatorname{det}^{\prime} \Delta^{k}$ denotes its zeta regularized determinant, ignoring the zero eigenvalues.

Recall that $H^{*}(M ; \mathbb{F})$ canonically identifies with the harmonic forms and thus inherits a Hermitian metric from $g$ and $\mu$. Let us write $\|\cdot\|_{\text {Hodge }}^{\mathbb{F}, g, \mu}$ for the induced Hermitian metric on $\operatorname{det} H^{*}(M ; \mathbb{F})$. The Ray-Singer metric is the Hermitian metric

$$
\|\cdot\|_{\mathrm{RS}}^{\mathbb{F}, g, \mu}:=T_{\mathrm{an}}(\nabla, g, \mu) \cdot\|\cdot\|_{\text {Hodge }}^{\mathbb{F}, g, \mu}
$$

on $\operatorname{det} H^{*}(M ; \mathbb{F})$, see [2].

Suppose $\mathbb{K}$ is $\mathbb{R}$ or $\mathbb{C}$. Let $V$ be a finite-dimensional vector space over $\mathbb{K}$. Recall that every representation of the fundamental group $\rho: \Gamma \rightarrow \mathrm{GL}(V)$ induces a canonical vector bundle $F_{\rho}$ equipped with a canonical flat connection $\nabla_{\rho}$. They are obtained from the trivial bundle $\tilde{M} \times V \rightarrow \tilde{M}$ and the trivial connection by passing to the $\Gamma$ 
quotient spaces. Here $\tilde{M}$ is the canonical universal covering provided by the base point $x_{0}$. The $\Gamma$-action is the diagonal action of deck transformations on $\widetilde{M}$ and the action $\rho$ on $V$. The fiber of $F_{\rho}$ over $x_{0}$ identifies canonically with $V$. The holonomy representation determines a right $\Gamma$-action on the fiber of $F_{\rho}$ over $x_{0}$, ie, an anti homomorphism $\Gamma \rightarrow \operatorname{GL}(V)$. When composed with the inversion in GL $(V)$ we get back the representation $\rho$. The pair $\left(F_{\rho}, \nabla_{\rho}\right)$ will be denoted by $\mathbb{F}_{\rho}$.

A Hermitian metric on $F_{\rho}$ induces a Hermitian metric $\|\cdot\|_{x_{0}}^{\mu}$ on $(\operatorname{det} V)^{-\chi(M)}$. Moreover, on $\operatorname{det} H^{*}\left(M ; \mathbb{F}_{\rho}\right)=\operatorname{det} H^{*}(M ; \rho)$ we have the Ray-Singer metric $\|\cdot\|_{\mathrm{RS}}^{\mathbb{F}_{\rho}, g, \mu}$. Suppose $\mathfrak{e}^{*} \in \mathfrak{E u l}_{X_{0}}(M ; \mathbb{R})$ is a co-Euler structure. Choose $\alpha$ so that $\mathfrak{e}^{*}=[g, \alpha]$; see Remark 3.7. Recall the Kamber-Tondeur form $\omega\left(\nabla_{\rho}, \mu\right) \in \Omega^{1}(M ; \mathbb{R})$ from Section 2.3. Consider the Hermitian metric

$$
\|\cdot\|_{\mathrm{an}}^{\rho, \mathrm{e}^{*}}:=\|\cdot\|_{\mathrm{RS}}^{\mathbb{F}_{\rho}, g, \mu} \otimes\|\cdot\|_{x_{0}}^{\mu} \cdot e^{-S\left(\omega\left(\nabla_{\rho}, \mu\right), \alpha\right)}
$$

on $\operatorname{Det}_{x_{0}}(M ; \rho)$; see $(33)$ and Lemma 2.3.

Lemma 3.14 The Hermitian metric (37) only depends on $\rho$ and the co-Euler structure $\mathfrak{e}^{*}=[g, \alpha]$ represented by $g$ and $\alpha$.

Proof This follows from well-known anomaly formulas for the Ray-Singer metric [2]. We first keep $\mu$ fixed and consider two Riemannian metrics $g_{1}$ and $g_{2}$ as well as $\alpha_{1}$ and $\alpha_{2}$ so that $\left[g_{1}, \alpha_{1}\right]=\left[g_{2}, \alpha_{2}\right]=\mathfrak{e}^{*}$. In view of Lemma 2.3 this implies

$$
S\left(\omega, \alpha_{2}\right)-S\left(\omega, \alpha_{1}\right)=\int_{M} \omega \wedge \operatorname{cs}\left(g_{1}, g_{2}\right)
$$

for every closed one-form $\omega \in \Omega^{*}(M ; \mathbb{R})$. In this situation [2, Theorem 0.1$]$ tells, with our notation,

$$
\log \left(\frac{\|\cdot\|_{\mathrm{RS}}^{\mathbb{F}_{\rho}, g_{2}, \mu}}{\|\cdot\|_{\mathrm{RS}}^{\mathbb{F}_{\rho}, g_{1}, \mu}}\right)^{2}=2 \int_{M} \omega\left(\nabla_{\rho}, \mu\right) \wedge \operatorname{cs}\left(g_{1}, g_{2}\right) .
$$

Together with (38) we obtain

$$
\begin{aligned}
\log \frac{\|\cdot\|_{\mathrm{RS}}^{\mathbb{F}_{\rho}, g_{2}, \mu}}{\|\cdot\|_{\mathrm{RS}}^{\mathbb{F}_{\rho}, g_{1}, \mu}} \otimes\|\cdot\|_{x_{0}}^{\mu} \cdot e^{-S\left(\omega\left(\nabla_{\rho}, \mu\right), \alpha_{2}\right)} \\
\quad=\int_{M}^{\mu} \omega\left(\nabla_{\rho}, \mu\right) \wedge \operatorname{cs}\left(g_{1}, g_{2}\right)-S\left(\omega\left(\nabla_{\rho}, \mu\right), \alpha_{2}\right)+S\left(\omega\left(\nabla_{\rho}, \mu\right), \alpha_{1}\right)=0 .
\end{aligned}
$$

Thus (37) does not depend on the representative of $\mathfrak{e}^{*}$. 
Next we keep $g$ and $\alpha$ fixed and consider two Hermitian metrics $\mu_{1}$ and $\mu_{2}$. Then from (20) we have $\omega\left(\nabla_{\rho}, \mu_{2}\right)-\omega\left(\nabla_{\rho}, \mu_{1}\right)=-\frac{1}{2} d \log V\left(\mu_{1}, \mu_{2}\right)$ and Lemma 2.3 implies

$$
\begin{aligned}
S\left(\omega\left(\nabla_{\rho}, \mu_{2}\right), \alpha\right)- & S\left(\omega\left(\nabla_{\rho}, \mu_{1}\right), \alpha\right)=-\frac{1}{2} S\left(d \log V\left(\mu_{1}, \mu_{2}\right), \alpha\right) \\
& =-\frac{1}{2} \chi(M) \log V\left(\mu_{1}, \mu_{2}\right)\left(x_{0}\right)+\frac{1}{2} \int_{M} \log V\left(\mu_{1}, \mu_{2}\right) E(g) .
\end{aligned}
$$

In this situation [2, Theorem 0.1$]$ states, with our notation,

$$
\log \left(\frac{\|\cdot\|_{\mathrm{RS}}^{\mathbb{F}_{\rho}, g, \mu_{2}}}{\|\cdot\|_{\mathrm{RS}}^{\mathbb{F}_{\rho}, g, \mu_{1}}}\right)^{2}=\int_{M} \log V\left(\mu_{1}, \mu_{2}\right) E(g) .
$$

Together with (39) and since $\left(\|\cdot\|_{x_{0}}^{\mu_{2}} /\|\cdot\|_{x_{0}}^{\mu_{1}}\right)^{2}=\left(V\left(\mu_{1}, \mu_{2}\right)\left(x_{0}\right)\right)^{-\chi(M)}$ we obtain

$$
\begin{aligned}
& \log \left(\frac{\|\cdot\|_{\mathrm{RS}}^{\mathbb{F}_{\rho}, g, \mu_{2}} \otimes\|\cdot\|_{x_{0}}^{\mu_{2}} \cdot e^{-S\left(\omega\left(\nabla_{\rho}, \mu_{2}\right), \alpha\right)}}{\|\cdot\|_{\mathrm{RS}}^{\mathbb{F}_{\rho}, g, \mu_{1}} \otimes\|\cdot\|_{x_{0}}^{\mu_{1}} \cdot e^{-S\left(\omega\left(\nabla_{\rho}, \mu_{1}\right), \alpha\right)}}\right)^{2}=\int_{M} \log V\left(\mu_{1}, \mu_{2}\right) E(g)- \\
& \quad-\chi(M) \log V\left(\mu_{1}, \mu_{2}\right)\left(x_{0}\right)-2 S\left(\omega\left(\nabla_{\rho}, \mu_{2}\right), \alpha\right)+2 S\left(\omega\left(\nabla_{\rho}, \mu_{1}\right), \alpha\right)=0 .
\end{aligned}
$$

Hence (37) is independent of $\mu$.

For $\beta \in H^{n-1}\left(M ; \mathcal{O}_{M}\right)$ we have

$$
\|\cdot\|_{\mathrm{an}}^{\rho, \mathrm{e}^{*}+\beta}=\|\cdot\|_{\mathrm{an}}^{\rho, \mathrm{e}^{*}} \cdot e^{\left\langle\log |[\operatorname{det} \circ \rho]|, \mathrm{PD}^{-1}(\beta)\right\rangle}
$$

(Lemma 2.3(i)) where $\log |[\operatorname{det} \circ \rho]| \in H^{1}(M ; \mathbb{R})$ is the Kamber-Tondeur class corresponding to the homomorphism $\log |[\operatorname{det} \circ \rho]|: H_{1}(M ; \mathbb{R}) \rightarrow \mathbb{R}$ as in Remark 2.1.

Definition 3.15 (Modified Ray-Singer metric) The Hermitian metric $\|\cdot\|_{\text {an }}^{\rho, e^{*}}$ on $\operatorname{Det}_{x_{0}}(M ; \rho)$ will be referred to as the modified Ray-Singer metric; see (33) and (37).

Remark 3.16 Suppose $n$ is odd and recall the canonical co-Euler structure $\mathfrak{e}_{\text {can }}^{*}$ in $\mathfrak{E u}^{*}(M ; \mathbb{R})$. Clearly, in this situation $\|\cdot\|_{\text {an }}^{\rho, \mathfrak{e}_{\text {can }}^{*}}=\|\cdot\|_{\mathrm{RS}}^{\mathbb{F}_{\rho}, g, \mu}$. From Lemma 3.14 we recover the well-known fact, that $\|\cdot\|_{\mathrm{RS}}^{\mathbb{F}_{\rho}, g, \mu}$ is independent of $g$ and $\mu$, provided $n$ is odd.

The Bismut-Zhang theorem [2, Theorem 0.2] can now be reformulated as a statement comparing two topological invariants, one defined with the help of analysis, and one defined with the help of a triangulation. 
Theorem 3.17 (Bismut-Zhang) Let $\left(M, x_{0}\right)$ be a closed connected manifold with base point, and let $\rho: \pi_{1}\left(M, x_{0}\right) \rightarrow \mathrm{GL}(V)$ be a finite-dimensional real or complex representation. Suppose $\mathfrak{e} \in \mathfrak{E u l}_{x_{0}}(M ; \mathbb{Z})$ is an Euler structure and $\mathfrak{e}^{*} \in \mathfrak{E u l}_{x_{0}}^{*}(M ; \mathbb{R})$ a co-Euler structure. Then one has

$$
\left\|\tau_{\mathrm{comb}}^{\rho, \mathfrak{e}, \mathfrak{o}}\right\|_{\mathrm{an}}^{\rho, \mathrm{e}^{*}}=e^{\left\langle\log |[\operatorname{det} \mathrm{o} \rho]|, \mathbb{T}\left(\mathfrak{e}, \mathfrak{e}^{*}\right)\right\rangle} .
$$

Particularly, if $P(\mathfrak{e})=\mathfrak{e}^{*}$ then $\left\|\tau_{\text {comb }}^{\rho, \mathfrak{e}, \mathfrak{o}}\right\|_{a n}^{\rho, \mathfrak{e}^{*}}=1$.

For an alternative proof of the (original) Bismut-Zhang theorem; see also Burghelea, Friedlander and Kappeler [8].

Remark 3.18 If $H^{*}(M ; \rho)=0$ then $\operatorname{Det}_{x_{0}}(M ; \rho)=\mathbb{K}$; see Remark 3.11. In this case the modified Ray-Singer metric gives rise to a real number $\mathcal{T}_{\text {an }}^{\mathfrak{e}^{*}}(\rho):=\|1\|_{\text {an }}^{\rho, \mathfrak{e}^{*}}>0$. From the very definition we have

$$
\mathcal{T}_{\text {an }}^{\mathrm{e}^{*}}(\rho)=T_{\text {an }}\left(\nabla_{\rho}, g, \mu\right) \cdot e^{-S\left(\omega\left(\nabla_{\rho}, \mu\right), \alpha\right)}
$$

where $\nabla_{\rho}$ denotes the flat connection on the associated bundle $F_{\rho}, g$ is a Riemannian metric on $M, \mu$ is a Hermitian structure on $F_{\rho}$, and $\alpha$ is such that $[g, \alpha]=\mathfrak{e}^{*}$. Recall the number $\mathcal{T}_{\text {comb }}^{\mathfrak{e}, \mathfrak{o}}(\rho) \in \mathbb{K}^{*}$ from Remark 3.13. Assuming $P(\mathfrak{e})=\mathfrak{e}^{*}$ the Bismut-Zhang theorem tells

$$
1=\left\|\tau_{\text {comb }}^{\rho, \mathfrak{e}, \mathfrak{o}}\right\|_{\text {an }}^{\rho, \mathfrak{e}^{*}}=\left|\mathcal{T}_{\text {comb }}^{\mathfrak{e}, \mathfrak{o}}(\rho)\right|^{-1}\|1\|_{\text {an }}^{\rho, \mathfrak{e}^{*}}=\left|\mathcal{T}_{\text {comb }}^{\mathfrak{e}, \mathfrak{o}}(\rho)\right|^{-1} \mathcal{T}_{\text {an }}^{\mathfrak{e}^{*}}(\rho),
$$

that is $\left|\mathcal{T}_{\text {comb }}^{\mathfrak{e}, \mathfrak{o}}(\rho)\right|=\mathcal{T}_{\text {an }}^{\mathfrak{e}^{*}}(\rho)$.

\section{Torsion and representations}

Warning Following standard conventions in algebraic geometry [18] we reserve the name affine algebraic variety for an irreducible variety in the affine space, and the term algebraic set for any finite union of varieties. By a rational function on an algebraic set we simply mean a rational function on each irreducible component.

\subsection{Complex representations}

Suppose $\Gamma$ is a finitely presented group with generators $g_{1}, \ldots, g_{r}$ and relations $R_{i}\left(g_{1}, g_{2}, \ldots, g_{r}\right)=e, i=1, \ldots, p$, and $V$ be a complex vector space of dimension $N$. Let $\operatorname{Rep}(\Gamma ; V)$ be the set of linear representations of $\Gamma$ on $V$, ie, group homomorphisms $\rho: \Gamma \rightarrow \mathrm{GL}_{\mathbb{C}}(V)$. By identifying $V$ to $\mathbb{C}^{N}$ this set is in a natural way an algebraic set inside the space $\mathbb{C}^{r N^{2}+1}$ given by $p N^{2}+1$ equations. Precisely 
if $A_{1}, \ldots, A_{r}, z$ represent the coordinates in $\mathbb{C}^{r N^{2}+1}$ with $A:=\left(a^{i j}\right), a^{i j} \in \mathbb{C}$, so $A \in \mathbb{C}^{N^{2}}$ and $z \in \mathbb{C}$, then the equations defining $\operatorname{Rep}(\Gamma ; V)$ are

$$
\begin{aligned}
z \cdot \operatorname{det}\left(A_{1}\right) \cdot \operatorname{det}\left(A_{2}\right) \cdots \operatorname{det}\left(A_{r}\right) & =1 \\
R_{i}\left(A_{1}, \ldots, A_{r}\right) & =\mathrm{id}, \quad i=1, \ldots, p
\end{aligned}
$$

with each of the equalities $R_{i}$ representing $N^{2}$ polynomial equations.

Suppose $\Gamma=\pi_{1}\left(M, x_{0}\right), M$ a closed manifold. Denote by $\operatorname{Rep}_{0}^{M}(\Gamma ; V)$ the set of representations $\rho$ with $H^{*}(M ; \rho)=0$ and notice that they form a Zariski open set in $\operatorname{Rep}(\Gamma ; V)$. Denote the Zariski closure of this set by $\operatorname{Rep}^{M}(\Gamma ; V)$. This is again an algebraic set which depends only on the homotopy type of $M$, can be empty (for example if $\chi(M) \neq 0$ ) but in some interesting cases can be the full space $\operatorname{Rep}(\Gamma ; V)$; cf Section 4.3.

Definition 4.1 ( $V$-acyclicity) Given a complex vector space $V$ we say that the closed manifold $M$ is $V$-acyclic if there exist representations $\rho \in \operatorname{Rep}(\Gamma ; V)$ with $H^{*}(M ; \rho)=0$. Equivalently $\operatorname{Rep}^{M}(\Gamma ; V) \neq \varnothing$.

\subsection{The space of cochain complexes and Turaev functions}

Let $\left(k_{0}, k_{1}, \ldots, k_{n}\right)$ be a string of nonnegative integers which satisfy the following requirements:

$$
\begin{aligned}
& k_{0}-k_{1}+k_{2} \mp \cdots+(-1)^{n} k_{n}=0 \\
& k_{i}-k_{i-1}+k_{i-2} \mp \cdots+(-1)^{i} k_{0} \geq 0 \text { for any } i \leq n-1 \text {. }
\end{aligned}
$$

Denote by $\mathbb{D}\left(k_{0}, \ldots, k_{n}\right)$ the collection of cochain complexes of the form

$$
C=\left(C^{*}, d^{*}\right): 0 \rightarrow C^{0} \stackrel{d^{0}}{\longrightarrow} C^{1} \stackrel{d^{1}}{\longrightarrow} \cdots \stackrel{d^{n-2}}{\longrightarrow} C^{n-1} \stackrel{d^{n-1}}{\longrightarrow} C^{n} \rightarrow 0
$$

with $C^{i}:=\mathbb{C}^{k_{i}}$, and by $\mathbb{D}_{\mathrm{ac}}\left(k_{0}, \ldots, k_{n}\right) \subseteq \mathbb{D}\left(k_{0}, \ldots, k_{n}\right)$ the subset of acyclic complexes. The cochain complex $C$ is determined by the collection $\left\{d^{i}\right\}$ of linear maps $d^{i}: \mathbb{C}^{k_{i}} \rightarrow \mathbb{C}^{k_{i+1}}$. If $\mathbb{D}\left(k_{0}, \ldots, k_{n}\right)$ is regarded as the subset of $\left\{d^{i}\right\} \in \mathbb{L}\left(k_{0}, \ldots, k_{n}\right)=$ $\bigoplus_{i=0}^{n-1} L\left(\mathbb{C}^{k_{i}}, \mathbb{C}^{k_{i+1}}\right)^{10}$ which satisfy the quadratic equations

$$
d^{i+1} \cdot d^{i}=0
$$

then $\mathbb{D}\left(k_{0}, \ldots, k_{n}\right)$ is an affine algebraic set given by degree two homogeneous polynomials and $\mathbb{D}_{\mathrm{ac}}\left(k_{0}, \ldots, k_{n}\right)$ is a Zariski open set in $\mathbb{D}\left(k_{0}, \ldots, k_{n}\right)$. We denote its Zariski closure by $\hat{\mathbb{D}}_{\mathrm{ac}}\left(k_{0}, \ldots, k_{n}\right)$.

\footnotetext{
${ }^{10}$ We denote by $L(V, W)$ the space of linear maps from $V$ to $W$.
} 


\section{Proposition 4.2}

(i) $\mathbb{D}_{\mathrm{ac}}\left(k_{0}, \ldots, k_{n}\right)$ is a connected smooth quasialgebraic set of dimension

$$
k_{0} \cdot k_{1}+\left(k_{1}-k_{0}\right) \cdot k_{2}+\cdots+\left(k_{n-1}-k_{n-2}+\cdots \pm k_{0}\right) \cdot k_{n} .
$$

(ii) $\widehat{\mathbb{D}}_{\mathrm{ac}}\left(k_{0}, \ldots, k_{n}\right)$ is an irreducible algebraic set, hence an affine algebraic variety

Proof The map

$$
\pi_{0}: \mathbb{D}_{\mathrm{ac}}\left(k_{0}, \ldots, k_{n}\right) \rightarrow \operatorname{Emb}\left(C^{0}, C^{1}\right)
$$

which associates to $C \in \mathbb{D}_{\mathrm{ac}}\left(k_{0}, \ldots, k_{n}\right)$ the linear map $d^{0}$, is a bundle whose fiber is isomorphic to $\mathbb{D}_{\mathrm{ac}}\left(k_{1}-k_{0}, k_{2}, \ldots, k_{n}\right)$. Note that $\operatorname{Emb}\left(C^{0}, C^{1}\right)$ is connected, smooth and of dimension $k_{0} \cdot k_{1}$. By induction this implies that $\mathbb{D}_{\mathrm{ac}}\left(k_{0}, \ldots, k_{n}\right)$ is a connected smooth quasialgebraic set and the dimension is as claimed. This shows the first part. The second part follows from the well-known fact [17, page 21] that a complex algebraic set, whose nonsingular part is connected with respect to standard topology, must be irreducible.

For any cochain complex in $C \in \mathbb{D}_{\mathrm{ac}}\left(k_{0}, \ldots, k_{n}\right)$ denote by

$$
B^{i}:=\operatorname{img}\left(d^{i-1}\right) \subseteq C^{i}=\mathbb{C}^{k_{i}}
$$

and consider the short exact sequence

$$
0 \rightarrow B^{i} \stackrel{\text { inc }}{\longrightarrow} C^{i} \stackrel{d^{i}}{\longrightarrow} B^{i+1} \rightarrow 0
$$

Choose a bases $b_{i}$ for each $B_{i}$, and choose lifts $\bar{b}_{i+1}$ of $b_{i+1}$ in $C^{i}$ using $d^{i}$, ie, $d^{i}\left(\bar{b}_{i+1}\right)=b_{i+1}$. Clearly $\left\{b_{i}, \bar{b}_{i+1}\right\}$ is a base of $C^{i}$.

Consider the base $\left\{b_{i}, \bar{b}_{i+1}\right\}$ as a collection of vectors in $C^{i}=\mathbb{C}^{k_{i}}$ and write them as a columns of a matrix $\left[b_{i}, \bar{b}_{i+1}\right]$. Define the torsion of the acyclic complex $C$, by

$$
\mathfrak{t}(C):=(-1)^{N} \prod_{i=0}^{n} \operatorname{det}\left[b_{i}, \bar{b}_{i+1}\right]^{(-1)^{i}}
$$

where $(-1)^{N}$ is Turaev's sign; see Farber and Turaev [15] or appendix A. The result is independent of the choice of the bases $b_{i}$ and of the lifts $\bar{b}_{i}[24 ; 15]$ and leads to the function

$$
\mathfrak{t}: \mathbb{D}_{\mathrm{ac}}\left(k_{0}, \ldots, k_{n}\right) \rightarrow \mathbb{C} \backslash 0 .
$$

Turaev provided a simple formula for this function [32] which permits to recognize $\mathfrak{t}$ as the restriction of a rational function on $\widehat{\mathbb{D}}_{\mathrm{ac}}\left(k_{0}, \ldots, k_{n}\right)$. 
Following Turaev [32], one considers symbols $\alpha \equiv\left(\alpha_{0}, \alpha_{1}, \ldots, \alpha_{n}\right)$ with $\alpha_{i}$ subsets the set of integers $\left\{1,2, \ldots, k_{i}\right\}$ so that

$$
\begin{aligned}
\alpha_{n} & =\varnothing \\
\sharp\left(\alpha_{i}\right) & =k_{i+1}-\sharp\left(\alpha_{i+1}\right) .
\end{aligned}
$$

In Turaev [31] such an $\alpha$ is called a $\tau$-chain. There are, of course, only finitely many $\tau$-chains for a given collection $\left(k_{0}, \ldots, k_{n}\right)$ as above.

For any cochain complex $C \in \mathbb{D}\left(k_{0}, \ldots, k_{n}\right)$, and in fact for any element $d \equiv\left\{d^{i}\right\}$ in $\mathbb{L}\left(k_{0}, \ldots, k_{n}\right)$, a $\tau$-chain defines the collection of square matrices $A_{i}\left(d^{i}\right)$ representing the $\sharp\left(\alpha_{i}\right) \times\left(k_{i+1}-\sharp\left(\alpha_{i+1}\right)\right)$-minor of the matrix $d^{i}$ consisting of the columns whose indices are in $\alpha_{i}$ and the rows not in $\alpha_{i+1}$.

For $d \equiv\left\{d^{i}\right\} \in \mathbb{L}\left(k_{0}, \ldots, k_{n}\right)$ one considers the expression

$$
F_{\alpha}(d)=\epsilon(\alpha) \prod_{i=0}^{n}\left(\operatorname{det} A_{i}\left(d^{i}\right)\right)^{(-1)^{i+1}}
$$

where $\epsilon(\alpha)= \pm 1$ and is chosen according to the strategy explained by Turaev [32, Remark 2.4, page 9]. Clearly $F_{\alpha}$ is a rational function on $\mathbb{L}\left(k_{0}, \ldots, k_{n}\right)$.

The cochain complex is called $\alpha$-nondegenerate if all quantities $\operatorname{det} A_{i}\left(d^{i}\right)$ are nonzero. Turaev has shown that:

(i) For an $\alpha$-nondegenerate cochain complex $C \in \mathbb{D}_{\mathrm{ac}}\left(k_{0}, \ldots, k_{n}\right), \mathfrak{t}(C)=F_{\alpha}(d)$ where $d=\left\{d_{i}\right\}$ is collection of the differentials in the complex $C$ [32, Theorem 2.2].

(ii) If $U_{\alpha}$ denotes the subsets of $\mathbb{D}_{\mathrm{ac}}\left(k_{0}, \ldots, k_{n}\right)$ consisting $\alpha$-nondegenerate complexes then $U_{\alpha}$ is open, and $\bigcup_{\alpha} U_{\alpha}=\mathbb{D}_{\mathrm{ac}}\left(k_{0}, \ldots, k_{n}\right)$.

(iii) For any $\tau$-chain $\alpha$ there exists a cochain complex $C \in \mathbb{D}_{\mathrm{ac}}\left(k_{0}, \ldots, k_{n}\right)$ which is $\alpha$-nondegenerate.

Using (i)-(iii) and the irreducibility of $\widehat{\mathbb{D}}_{\mathrm{ac}}\left(k_{0}, \ldots, k_{n}\right)$ we conclude: every $F_{\alpha}$ restricts to a rational function on $\widehat{\mathbb{D}}_{\mathrm{ac}}\left(k_{0}, \ldots, k_{n}\right)$; as rational functions, these restrictions agree, are regular on $\mathbb{D}_{\mathrm{ac}}\left(k_{0}, \ldots, k_{n}\right)$, and they coincide with (42) on $\mathbb{D}_{\mathrm{ac}}\left(k_{0}, \ldots, k_{n}\right)$. Therefore (42) is the restriction of a rational function on $\widehat{\mathbb{D}}_{\mathrm{ac}}\left(k_{0}, \ldots, k_{n}\right)$ with zeros and poles contained in $\widehat{\mathbb{D}}_{\mathrm{ac}}\left(k_{0}, \ldots, k_{n}\right) \backslash \mathbb{D}_{\mathrm{ac}}\left(k_{0}, \ldots, k_{n}\right)$.

Consider now a smooth triangulation $\tau$ of $M$ whose set of simplices of dimension $q$ is denoted by $\mathcal{X}_{q}$ and the collection of integers $k_{i}=\sharp\left(\mathcal{X}_{i}\right) \cdot \operatorname{dim} V$ where $V$ is a fixed complex vector space. Let $\mathfrak{e} \in \mathfrak{E u l}_{x_{0}}(M ; \mathbb{Z})$ be an Euler structure, $\mathfrak{o}$ a cohomology 
orientation, and suppose that $M$ is $V$-acyclic. Then the integers $\left(k_{0}, \ldots, k_{n}\right)$ satisfy (40) and (41). As in Section 3.3, choose a collection of paths $\pi_{\mathfrak{e}} \equiv\left\{\pi_{x}\right\}$ from $x_{0}$ to the barycenters $x$, so that $\mathfrak{e}=\left[X_{\tau}, c\right]$ where $c$ is the Euler chain defined by $\left\{\pi_{x}\right\}$. Choose also an ordering $o$ of the barycenters (zeros of $X_{\tau}$ ) compatible with $\mathfrak{o}$, and a framing $\epsilon$ of $V$.

Consider the chain complex $\left(C_{\tau}^{*}(M ; \rho), d_{\tau}(\rho)\right)$ associated with the triangulation $\tau$ which computes the cohomology $H^{*}(M ; \rho)$. Using $\pi_{x}, o$ and $\epsilon$ one can identify $C_{\tau}^{q}(M ; \rho)$ with $\mathbb{C}^{k_{q}}$. We obtain in this way a map

$$
t_{\pi_{\mathfrak{e}}, o, \epsilon}: \operatorname{Rep}(\Gamma ; V) \rightarrow \mathbb{D}\left(k_{0}, \ldots, k_{n}\right)
$$

which sends $\operatorname{Rep}^{M}(\Gamma ; V) \backslash \Sigma(M)$ to $\mathbb{D}_{\mathrm{ac}}\left(k_{0}, \ldots, k_{n}\right)$. Here $\Sigma(M) \subseteq \operatorname{Rep}(\Gamma ; V)$ denotes the Zariski closed subset of representations $\rho$ which are not local minima for the function $\rho \mapsto \sum_{i=0}^{n} \operatorname{dim} H^{i}(M ; \rho)$. A look at the explicit definition of $d_{\tau}(\rho)$ implies that $t_{\pi_{\mathfrak{e}}, o, \epsilon}$ is actually a regular map between two algebraic sets. Change of $\epsilon$, $\pi_{\mathfrak{e}}$ and $o$ changes the map $t_{\pi_{\mathfrak{e}}, o, \epsilon}$. Since we are in the case $H^{*}(M ; \rho)=0$ we easily recognize that the composition $\mathfrak{t} \cdot t_{\pi_{\mathfrak{e}}, o, \epsilon}$, when restricted to $\operatorname{Rep}^{M}(\Gamma ; V) \backslash \Sigma(M)$, is exactly the Milnor-Turaev torsion $\mathcal{T}_{\text {comb }}^{\mathfrak{e}, \mathfrak{o}}$ (Remark 3.13) hence independent of all these choices $\pi_{\mathfrak{e}}, o, \epsilon$.

We summarize these observations in the following theorem.

Theorem 4.3 Let $M$ be a closed manifold, $x_{0}$ a base point, $\Gamma:=\pi_{1}\left(M, x_{0}\right)$ the fundamental group, $\mathfrak{e} \in \mathfrak{E u l}_{x_{0}}(M ; \mathbb{Z})$ an Euler structure, $\mathfrak{o}$ a cohomology orientation, and let $V$ be a finite-dimensional complex vector space. Consider the Milnor-Turaev torsion $\mathcal{T}_{\text {comb }}^{\mathfrak{e}, \mathfrak{o}}: \operatorname{Rep}^{M}(\Gamma ; V) \backslash \Sigma(M) \rightarrow \mathbb{C} \backslash 0$.

(i) $\mathcal{T}_{\text {comb }}^{\mathfrak{e}, \mathfrak{o}}$ is a rational function on the algebraic set $\operatorname{Rep}^{M}(\Gamma ; V)$ whose poles and zeros are contained in $\Sigma(M)$.

(ii) The absolute value of $\mathcal{T}_{\text {comb }}^{\mathfrak{e}, \mathfrak{o}}$ is the modified Ray-Singer torsion $\mathcal{T}_{\text {an }}^{\mathfrak{e}^{*}}$, where $\mathfrak{e}^{*}=P(\mathfrak{e}) \in \mathfrak{E u l}_{x_{0}}^{*}(M ; \mathbb{R})$; see Remark 3.18. More precisely, for a representation $\rho \in \operatorname{Rep}^{M}(\Gamma ; V) \backslash \Sigma(M)$ one has

$\left|\mathcal{T}_{\text {comb }}^{\mathfrak{e}, \mathfrak{o}}(\rho)\right|=\mathcal{T}_{\text {an }}^{\mathfrak{e}^{*}}(\rho)=T_{\text {an }}\left(\nabla_{\rho}, g, \mu, \alpha\right)=T_{\text {an }}\left(\nabla_{\rho}, g, \mu\right) \cdot e^{-S\left(\omega\left(\nabla_{\rho}, \mu\right), \alpha\right)}$

where $\left(F_{\rho}, \nabla_{\rho}\right)$ denotes the flat complex vector bundle associated to the representation $\rho, g$ is a Riemannian metric on $M, \mu$ is a Hermitian structure on $F_{\rho}, \omega\left(\nabla_{\rho}, \mu\right)$ is the Kamber-Tondeur form, and $\alpha$ is such that the pair $(g, \alpha)$ represents the co-Euler structure $\mathfrak{e}^{*}=P(\mathfrak{e})$. 
(iii) For $\sigma \in H_{1}(M ; \mathbb{Z})$, and $\rho \in \operatorname{Rep}^{M}(\Gamma ; V) \backslash \Sigma(M)$ we have $\mathcal{T}_{\text {comb }}^{\mathfrak{e}+\sigma, \mathfrak{o}}(\rho)=\mathcal{T}_{\text {comb }}^{\mathfrak{e}, \mathfrak{o}}(\rho) \cdot[\operatorname{det} \circ \rho](\sigma) \quad$ and $\quad \mathcal{T}_{\text {comb }}^{\mathfrak{e},-\mathfrak{o}}(\rho)=(-1)^{\operatorname{dim} V} \cdot \mathcal{T}_{\text {comb }}^{\mathfrak{e}, \mathfrak{o}}(\rho)$ where $[\operatorname{det} \circ \rho]: H_{1}(M ; \mathbb{Z}) \rightarrow \mathbb{C} \backslash 0$ is the homomorphism induced from the map $\operatorname{det} \circ \rho: \Gamma \rightarrow \mathbb{C} \backslash 0$.

\subsection{Milnor-Turaev torsion for mapping tori}

Let $N$ be a closed connected manifold and $\varphi: N \rightarrow N$ a diffeomorphism. Define the mapping torus $M=N_{\varphi}$ by gluing the boundaries of $N \times I$ with the help of $\varphi$, more precisely identifying $(x, 1)$ with $(\varphi(x), 0)$. The manifold $M$ comes with a projection $p: M \rightarrow S^{1}$, and an embedding $N \rightarrow M, x \mapsto(x, 0)$. Note that $\pi_{1}\left(S^{1}, 0\right)=\mathbb{Z}$. Let $\mathbb{K}$ be a field of characteristics zero, and suppose $V$ is a finite-dimensional vector space over $\mathbb{K}$. For $\rho \in \operatorname{Rep}(\mathbb{Z} ; V)=\mathrm{GL}(V)$ define

$$
P_{\varphi}^{k}(\rho):=\operatorname{det}\left(\varphi^{*} \circ \rho_{*}-\mathrm{id}: H^{k}(N ; V) \rightarrow H^{k}(N ; V)\right)
$$

and the Lefschetz zeta function

$$
\zeta_{\varphi}(\rho):=\frac{\prod_{k \text { even }} P_{\varphi}^{k}(\rho)}{\prod_{k \text { odd }} P_{\varphi}^{k}(\rho)} .
$$

This is a rational function $\zeta_{\varphi}: \mathrm{GL}(V) \rightarrow \mathbb{K}$.

Choose a base point $x_{0} \in M$ with $p\left(x_{0}\right)=0 \in S^{1}$. Every representation $\rho \in \operatorname{Rep}(\mathbb{Z} ; V)$ gives rise to a representation $p^{*} \rho$ of $\pi_{1}\left(M, x_{0}\right)$ on $V$. It is not hard to see that $H^{*}\left(M ; p^{*} \rho\right)=0$ if and only if $P_{\varphi}^{k}(\rho) \neq 0$ for all $k$. Indeed, we have a long exact sequence, the Wang sequence:

(43) $\cdots \rightarrow H^{*}\left(M ; p^{*} \rho\right) \rightarrow H^{*}(N ; V) \stackrel{\varphi^{*} \circ \rho_{*}-\mathrm{id}}{\longrightarrow} H^{*}(N ; V) \rightarrow H^{*+1}\left(M ; p^{*} \rho\right) \rightarrow \cdots$

Recall that this sequence is derived from the long exact sequence of the pair $(M, N)$, and the isomorphism $H^{*}\left(M, N ; p^{*} \rho\right)=H^{*}(N \times I, N \times \partial I ; V)=H^{*-1}(N ; V)$. The latter is the Thom isomorphism with the standard orientation on $I$, and the first comes from a trivialization of the coefficient system on $N \times I$ from $N \times\{0\}$ upwards.

Let $\omega:=p^{*} d t \in \Omega^{1}(M)$. The space of vector fields $X$ on $M$ satisfying $\omega(X)<0$ is contractible and hence defines an Euler structure $\mathfrak{e} \in \mathfrak{E u l}(M ; \mathbb{Z})$; cf Theorem 3.10(ii).

Recall that a finite-dimensional long exact sequence of $\mathbb{K}$ vector spaces

$$
\cdots \rightarrow A^{n} \rightarrow B^{n} \rightarrow C^{n} \rightarrow A^{n+1} \rightarrow \cdots
$$


induces a canonical isomorphism of graded determinant lines

$$
u: \operatorname{det}\left(A^{*}\right) \otimes \operatorname{det}\left(C^{*}\right) \rightarrow \operatorname{det}\left(B^{*}\right), \quad a \otimes b \rightarrow u(a \otimes b) .
$$

Indeed, if one regards this sequence as an acyclic cochain complex

$$
0 \rightarrow D^{0} \rightarrow D^{1} \cdots \rightarrow D^{n} \rightarrow D^{n+1} \rightarrow \cdots
$$

one obtains a canonical isomorphism $\operatorname{det}\left(D^{*}\right) \rightarrow \mathbb{K}$; see (62) in the appendix. (Since the complex is acyclic the Turaev sign correction does not appear.) In view of the identification of $\operatorname{det}\left(D^{*}\right)$ with $\operatorname{det}\left(A^{*}\right) \otimes\left(\operatorname{det}\left(B^{*}\right)\right)^{-1} \otimes \operatorname{det}\left(C^{*}\right)$ one obtains the canonical isomorphism (44). If in addition $B^{*}=C^{*}$ we obtain a canonical isomorphism $\operatorname{det}\left(A^{*}\right) \rightarrow \mathbb{K}, a \rightarrow \operatorname{tr}(u(a \otimes \cdot))$. Applying this to the Wang exact sequence for the trivial one-dimensional real representation

$$
\cdots \rightarrow H^{*}(M ; \mathbb{R}) \rightarrow H^{*}(N ; \mathbb{R}) \rightarrow H^{*}(N ; \mathbb{R}) \rightarrow H^{*+1}(M ; \mathbb{R}) \rightarrow \cdots
$$

we obtain a canonical isomorphism $\operatorname{det} H^{*}(M ; \mathbb{R})=\mathbb{R}$ hence a canonical cohomology orientation $\mathfrak{o}$.

The rest of this section is dedicated to the proof of the following proposition; see also Fried [16].

Proposition 4.4 Let $\mathfrak{e}$ and $\mathfrak{o}$ denote the canonical Euler structure and cohomology orientation on the mapping torus $M$ introduced above. If $H^{*}\left(M ; p^{*} \rho\right)=0$, then $\mathcal{T}_{\text {comb }}^{\mathfrak{e}, \mathfrak{o}}\left(p^{*} \rho\right)=(-1)^{\operatorname{dim}(V) \cdot z_{\varphi}} \zeta_{\varphi}(\rho)$ where

$$
z_{\varphi}=\sum_{q} \operatorname{dim} H^{q}(N ; \mathbb{R}) \cdot \operatorname{dim} \operatorname{ker}\left(\varphi^{*}-\mathrm{id}: H^{q}(N ; \mathbb{R}) \rightarrow H^{q}(N ; \mathbb{R})\right) .
$$

To get a hold on the Euler structure $\mathfrak{e}$ we start with the following lemma.

Lemma 4.5 Suppose $\tilde{\tau}$ is a smooth triangulation of $M$ such that $N$ is a subcomplex. For every simplex $\sigma \in \tilde{\tau}$ let $\pi_{\sigma}$ be a path in $N \times[0,1)$ considered as path in $M$ from the base point $x_{0} \in N$ to the barycenter $x_{\sigma}$. Consider the Euler structure $\tilde{\mathfrak{e}}=\left[X_{\tilde{\tau}}, c_{\tilde{\tau}}\right]$ where $c_{\tilde{\tau}}:=\sum_{\sigma \in \tilde{\tau}}(-1)^{\operatorname{dim} \sigma_{\pi}} \pi_{\sigma}$. If $\mathfrak{e}$ is the Euler structure described above then $\left[\operatorname{det} \circ p^{*} \rho\right](\tilde{\mathfrak{e}}-\mathfrak{e})=1$. Particularly, $\mathcal{T}_{\text {comb }}^{\mathfrak{e}, \mathfrak{o}}\left(p^{*} \rho\right)=\mathcal{T}_{\text {comb }}^{\tilde{\mathfrak{e}} \mathfrak{o}}\left(p^{*} \rho\right)$. The same statement remains true when $\tilde{\tau}$ is a smooth cell structure as in Remark 2.7.

Proof Let $X$ be a vector field on $M$ with $\omega(X)<0$ and recall that $\mathfrak{e}=[X, 0]$. Since $N$ is a subcomplex we find a sufficiently small $\epsilon>0$ such that $N \times[1-\epsilon, 1) \subseteq M$ does not contain barycenters of simplices of $\tilde{\tau}$. In view of P2 in Section 2.7 we may also assume $\omega\left(X_{\tilde{\tau}}\right)<0$ on $N \times[1-\epsilon, 1)$. Let us write $N_{\epsilon}:=N \times\{1-\epsilon\} \subseteq M$. We thus 
find a homotopy $\mathbb{X}$ from $X$ to $X_{\tilde{\tau}}$ which has the property that $\omega\left(\mathbb{X}_{t}\right)<0$ on $N_{\epsilon}$ for all $t \in I$. We conclude that $\operatorname{cs}\left(X, X_{\tilde{\tau}}\right)$ has a representative supported in $M \backslash N_{\epsilon}$. Since $\tilde{\mathfrak{e}}-\mathfrak{e} \in H_{1}(M ; \mathbb{Z})$ is represented by $c_{\tilde{\tau}}-\operatorname{cs}\left(X, X_{\tilde{\tau}}\right)$ it too has a representative supported in $M \backslash N_{\epsilon}$. We conclude $p_{*}(\tilde{\mathfrak{e}}-\mathfrak{e})=0 \in H_{1}\left(S^{1} ; \mathbb{Z}\right)$, and hence $\left[\operatorname{det} \circ p^{*} \rho\right](\widetilde{\mathfrak{e}}-\mathfrak{e})=1$. The last assertion follows from Theorem 4.3(iii).

Lemma 4.6 Let $\varphi_{i}: N \rightarrow N$ be diffeomorphisms, let $\mathfrak{e}_{i}$ resp. $\mathfrak{o}_{i}$ denote the Euler structure resp. cohomology orientation on $N_{\varphi_{i}}$ introduced above, and suppose $H^{*}\left(N_{\varphi_{i}} ; p^{*} \rho\right)=0, i=a, b$. Then

$$
\mathcal{T}_{\mathrm{comb}}^{N_{\varphi_{b}, \mathfrak{e}_{b}, \mathfrak{o}_{b}}}\left(p^{*} \rho\right) \cdot \mathcal{T}_{\mathrm{comb}}^{N_{\varphi_{a}, \mathfrak{e}_{a}, \mathfrak{o}_{a}}}\left(p^{*} \rho\right)^{-1}=(-1)^{\operatorname{dim}(V) \cdot\left(z_{\varphi_{b}}-z_{\varphi_{a}}\right)} \zeta_{\varphi_{b}}(\rho) \cdot \zeta_{\varphi_{a}}(\rho)^{-1}
$$

Proof We may assume, by changing $\varphi_{i}$ up to isotopy, that there are smooth triangulations $\tau_{0}$ and $\tau_{1}$ of $N$ such that both $\varphi_{a}$ and $\varphi_{b}$ are simplicial. Let $\tau$ be a smooth triangulation on $N \times[0,1]$ such that $N \times\{0\}$ and $N \times\{1\}$ are subcomplexes and the triangulation induced by $\tau$ on $N \times\{0\}$ resp. $N \times\{1\}$ is exactly $\tau_{0}$ resp. $\tau_{1}$. Let $\tilde{\tau}_{a}$ and $\tilde{\tau}_{b}$ denote the cell structures on $N_{\varphi_{a}}$ and $N_{\varphi_{b}}$ induced by $\tau \cdot{ }^{11}$ Note that $\tilde{\tau}_{a}$ and $\tilde{\tau}_{b}$ are smooth cell structures (of the type considered in Remark 2.7) but not smooth triangulations. The characteristic maps are smooth embeddings of compact affine simplices; they satisfy the axioms of a cell complex but not of a triangulation. Also recall that the torsion $\mathcal{T}_{\text {comb }}^{N_{\varphi_{i}, \mathfrak{e}, \mathfrak{o}}}\left(p^{*} \rho\right)$ can be computed using any cell structure [24]. A simple inspection of the Turaev sign corrections indicates that such correction can be performed mutatis-mutandis in this more general case.

We denote by $\mathcal{X}, \mathcal{X}_{0}$ and $\mathcal{X}_{1}$ the set of simplices (cells) of $\tau, \tau_{0}$ and $\tau_{1}$. The cells of both $\tilde{\tau}_{a}$ and $\tilde{\tau}_{b}$ are indexed by $\mathcal{X} \backslash \mathcal{X}_{0}$. Hence the graded vector spaces $C_{\tilde{\tau}_{a}}^{*}\left(N_{\varphi_{a}} ; p^{*} \rho\right)$ and $C_{\tilde{\tau}_{b}}^{*}\left(N_{\varphi_{b}} ; p^{*} \rho\right)$ are tautologically the same. Moreover the relative cochain complexes $C_{\tilde{\tau}_{a}}^{*}\left(N_{\varphi_{a}}, N ; p^{*} \rho\right), C_{\tilde{\tau}_{b}}^{*}\left(N_{\varphi_{b}}, N ; p^{*} \rho\right)$ and $C_{\tau}^{*}\left(N \times I, N \times \partial I ; p^{*} \rho\right)$ are tautologically the same; their cells are indexed by $\mathcal{X} \backslash\left\{\mathcal{X}_{0} \cup \mathcal{X}_{1}\right\}$ and the differentials coincide.

The two short exact sequences of cochain complexes

$$
C_{\tau}^{*}\left(N \times I, N \times \partial I ; p^{*} \rho\right)=C_{\tilde{\tau}_{i}}^{*}\left(N_{\varphi_{i}}, N ; p^{*} \rho\right) \rightarrow C_{\widetilde{\tau}_{i}}^{*}\left(N_{\varphi_{i}} ; p^{*} \rho\right) \rightarrow C_{\tau_{1}}^{*}(N ; V)
$$

\footnotetext{
${ }^{11}$ Observe that if $K$ is a simplicial complex with $L_{1}$ and $L_{2}$ two disjoint subcomplexes and $\varphi: L_{1} \rightarrow$ $L_{2}$ a simplicial map the quotient space $K_{\varphi}$ obtained by identifying $x \in L_{1}$ to $\varphi(x) \in L_{2}$ is a cell complex whose cells are in bijective correspondence to the open simplices of $K \backslash L_{1}$.
} 
$i=a, b$, give rise to commuting fusion diagrams as explained in Lemma A.1 in the appendix. These make up the upper half of the following diagram:

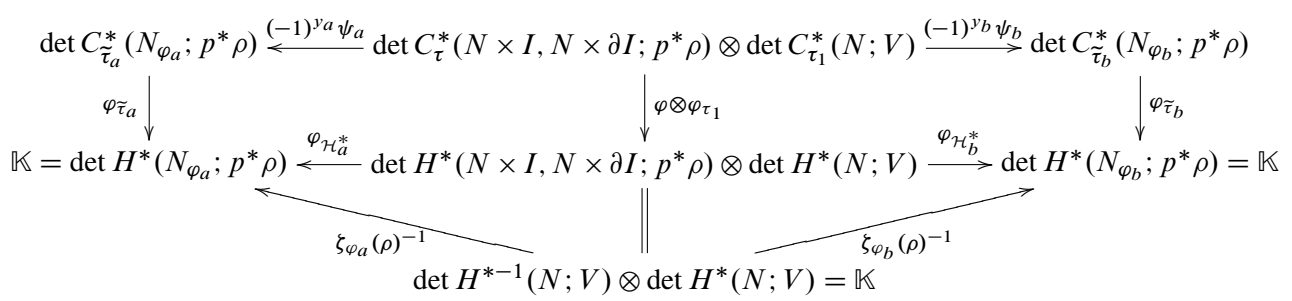

Note that Lemma A.1 is here applied to short exact sequences where the middle complex $C_{1}^{*}$ is acyclic. Therefore Lemma A.1 also implies

$$
y_{a} \equiv y_{b} \quad \bmod 2 .
$$

The vertical identification in the lower part of the diagram is explained in the text below (43). Because of (43) the lower triangles commute too.

For every simplex $\sigma \in \mathcal{X} \backslash \mathcal{X}_{0}$ let $\pi_{\sigma}$ be a path in $N \times[0,1)$ from the base point $x_{0} \in N$ to the barycenter $x_{\sigma}$ and consider the Euler chains $c_{\tau_{a}}$ resp. $c_{\tau_{b}}$ defined by the collections of paths $\left\{\pi_{\sigma}\right\}$ viewed in $N_{\varphi_{a}}$ resp. $N_{\varphi_{b}}$ (by the formula $c_{\tilde{\tau}}:=\sum_{\sigma \in \tilde{\tau}}(-1)^{\operatorname{dim} \sigma_{\pi}} \pi_{\sigma}$.) Consider the diagram

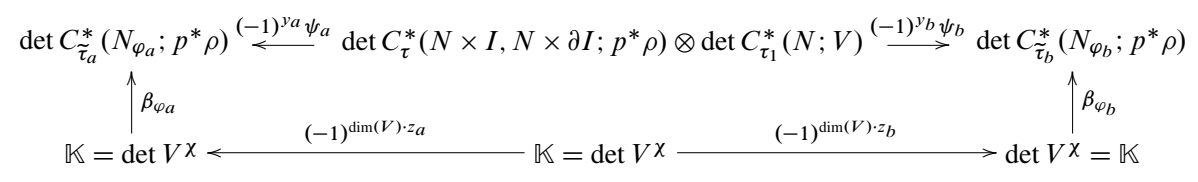

where $\beta_{\varphi_{a}}$ and $\beta_{\varphi_{b}}$ are induced from the homogeneous maps of degree $\chi=\chi\left(N_{\varphi_{a}}\right)=$ $\chi\left(N_{\varphi_{b}}\right)=0$ which associates to a frame of $V$ the equivalence class of bases induced from the frame, the collection of paths $\left\{\pi_{\sigma}\right\}$ and the ordering of the simplices in $\mathcal{X} \backslash \mathcal{X}_{0}$ representing the canonical cohomology orientation $\mathfrak{o}_{a}$ resp. $\mathfrak{o}_{b}$ of $N_{\varphi_{a}}$ resp. $N_{\varphi_{b}}$.

We would like to prove that the diagram is commutative. If so, in view of Lemma 4.5, Definition 3.12 and Remark 3.13, by putting together the two diagrams we conclude the result as stated.

It is easy to note the commutativity up to sign (independent on the representation $\rho$ ) of the second diagram, since the Euler structures $\mathfrak{e}_{a}$ resp. $\mathfrak{e}_{b}$ are realized by the same Euler chain given by $\left\{\pi_{\sigma}\right\}$; cf Lemma 4.5. We then conclude the result up to a sign independent of the representation $\rho$.

To decide about the sign we have to compare the orderings of $\mathcal{X} \backslash \mathcal{X}_{0}$ induced from the canonical cohomology orientation on $N_{\varphi_{a}}$ and on $N_{\varphi_{b}}$. To do this we consider the 
fusion diagram for the trivial one-dimensional real representation:

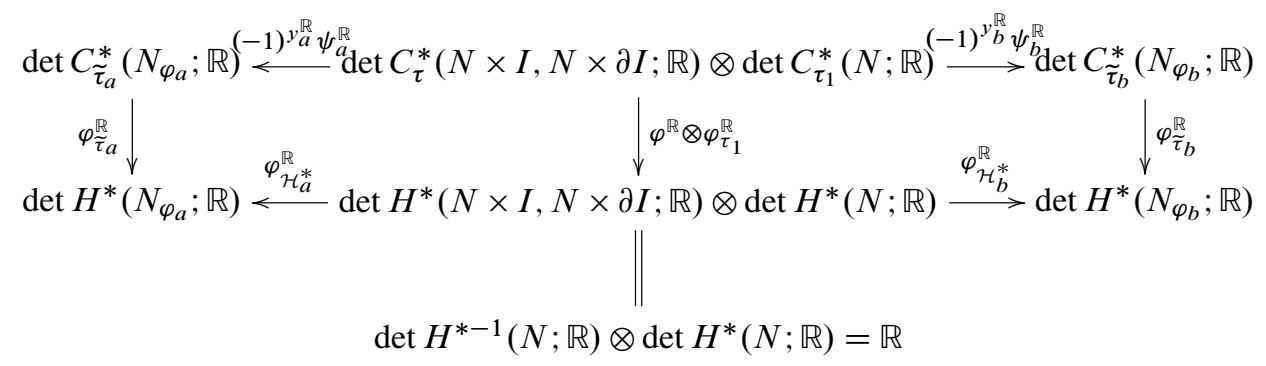

Let $o_{i}$ denote the equivalence class of orderings of $\mathcal{X} \backslash \mathcal{X}_{0}$ induced by the cohomology orientation $\mathfrak{o}_{i}, i=a, b$. The commutativity of the previous diagram tells

$$
\operatorname{sign}\left(o_{b} / o_{a}\right)=(-)^{y_{b}^{\mathbb{R}}-y_{a}^{\mathbb{R}}} .
$$

Diagram (46) will commute if and only if

$$
(-1)^{\operatorname{dim}(V) \cdot\left(z_{b}-z_{a}\right)}(-1)^{y_{b}-y_{a}} \operatorname{sign}\left(o_{b} / o_{a}\right)^{\operatorname{dim}(V)}=1 .
$$

In view of (45) and (47) it thus suffices to show

$$
y_{b}^{\mathbb{R}}-y_{a}^{\mathbb{R}} \equiv z_{b}-z_{a} \quad \bmod 2 .
$$

To establish this relation let us write for $i=a, b$

$$
\begin{aligned}
x_{i}^{q} & :=\operatorname{dim} \operatorname{ker}\left(\left(\varphi_{i}^{*}-\mathrm{id}\right): H^{q}(N ; \mathbb{R}) \rightarrow H^{q}(N ; \mathbb{R})\right) \\
f_{i, 1}^{q} & :=\operatorname{dim} \operatorname{img}\left(H^{q}\left(C_{\tau}^{*}(N \times I, N \times \partial I ; \mathbb{R})\right) \rightarrow H^{q}\left(C_{\tilde{\tau}_{i}}^{*}\left(N_{\varphi_{i}} ; \mathbb{R}\right)\right)\right) \\
f_{i, 2}^{q} & :=\operatorname{dimimg}\left(H^{q}\left(C_{\tilde{\tau}_{i}}^{*}\left(N_{\varphi_{i}} ; \mathbb{R}\right)\right) \rightarrow H^{q}\left(C_{\tau_{1}}^{*}(N ; \mathbb{R})\right)\right)
\end{aligned}
$$

where the homomorphisms in (49) and (50) are the ones from the long exact cohomology sequence associated with the short exact sequence of complexes

$$
0 \rightarrow C_{\tau}^{*}(N \times I, N \times \partial I ; \mathbb{R}) \rightarrow C_{\tilde{\tau}_{i}}^{*}\left(N_{\varphi_{i}} ; \mathbb{R}\right) \rightarrow C_{\tau_{1}}^{*}(N ; \mathbb{R}) \rightarrow 0 .
$$

Note that for $\left(\varphi_{i}^{*}-\mathrm{id}\right): H^{q}(N ; \mathbb{R}) \rightarrow H^{q}(N ; \mathbb{R})$, we have

$$
\operatorname{dim} \operatorname{ker}\left(\varphi_{i}^{*}-\mathrm{id}\right)=\operatorname{dim} \operatorname{coker}\left(\varphi_{i}^{*}-\mathrm{id}\right),
$$

and thus (43) gives

$$
f_{i, 2}^{q}=x_{i}^{q} \quad \text { and } \quad f_{i, 1}^{q}=x_{i}^{q-1}, \quad i=a, b .
$$


Let us also introduce

$$
\begin{aligned}
b_{0}^{q} & :=\operatorname{dim} B^{q}\left(C_{\tau}^{*}(N \times I, N \times \partial I ; \mathbb{R})\right) \\
b_{2}^{q} & :=\operatorname{dim} B^{q}\left(C_{\tau_{1}}^{*}(N ; \mathbb{R})\right)
\end{aligned}
$$

where $B^{q}$ denotes the space of boundaries in degree $q$. From Lemma A.1 and (51) we obtain

$$
\begin{array}{rl}
y_{b}^{\mathbb{R}}-y_{a}^{\mathbb{R}}=N & \left(C_{\tilde{\tau}_{b}}^{*}\left(N_{\varphi_{b}} ; \mathbb{R}\right)\right)-N\left(C_{\tilde{\tau}_{a}}^{*}\left(N_{\varphi_{a}} ; \mathbb{R}\right)\right) \\
& +\sum_{q}\left(f_{b, 1}^{q}-f_{a, 1}^{q}\right) b_{2}^{q}+\sum_{q}\left(f_{b, 2}^{q}-f_{b, 1}^{q}\right) b_{0}^{q+1} \\
=N & N\left(C_{\tilde{\tau}_{b}}^{*}\left(N_{\varphi_{b}} ; \mathbb{R}\right)\right)-N\left(C_{\tilde{\tau}_{a}}^{*}\left(N_{\varphi_{a}} ; \mathbb{R}\right)\right)+\sum_{q}\left(x_{b}^{q-1}-x_{a}^{q-1}\right)\left(b_{2}^{q}+b_{0}^{q}\right) .
\end{array}
$$

Let us further introduce

$$
\begin{aligned}
\alpha_{0}^{q} & :=\sum_{j \geq q} \operatorname{dim} C_{\tau}^{j}(N \times I, N \times \partial I ; \mathbb{R}) \\
\alpha_{i, 1}^{q}: & =\sum_{j \geq q} \operatorname{dim} C_{\tilde{\tau}_{i}}^{j}\left(N_{\varphi_{i}} ; \mathbb{R}\right) \quad i=a, b \\
\alpha_{2}^{q} & :=\sum_{j \geq q} \operatorname{dim} C_{\tau_{1}}^{j}(N ; \mathbb{R})
\end{aligned}
$$

and note that $\alpha_{a, 1}^{q}=\alpha_{b, 1}^{q}=\alpha_{0}^{q}+\alpha_{2}^{q}$. Finally set

$$
\begin{aligned}
\beta_{0}^{q} & :=\sum_{j \geq q} \operatorname{dim} H^{j}\left(C_{\tau}^{*}(N \times I, N \times \partial I ; \mathbb{R})\right) \\
\beta_{i, 1}^{q}: & =\sum_{j \geq q} \operatorname{dim} H^{j}\left(C_{\widetilde{\tau}_{i}}^{*}\left(N_{\varphi_{i}} ; \mathbb{R}\right)\right) \quad i=a, b \\
\beta_{2}^{q} & :=\sum_{j \geq q} \operatorname{dim} H^{j}\left(C_{\tau_{1}}^{*}(N ; \mathbb{R})\right) .
\end{aligned}
$$

Note that $\beta_{0}^{q}=\beta_{2}^{q-1}$ and $\beta_{2}^{q-1}-\beta_{2}^{q}=\operatorname{dim} H^{q-1}(N ; \mathbb{R})$ and hence

$$
\beta_{0}^{q}+\beta_{2}^{q} \equiv \operatorname{dim} H^{q-1}(N ; \mathbb{R}) \quad \bmod 2 .
$$

From (43) we find $\operatorname{dim} H^{j}\left(C_{\widetilde{\tau}_{i}}^{*}\left(N_{\varphi_{i}} ; \mathbb{R}\right)\right)=x_{i}^{j}+x_{i}^{j-1}, i=a, b$, and thus

$$
\beta_{i, 1}^{q} \equiv x_{i}^{q-1} \bmod 2 \quad i=a, b
$$


From this and $\alpha_{a, 1}^{q}=\alpha_{b, 1}^{q}=\alpha_{0}^{q}+\alpha_{2}^{q}$ we obtain

$$
\begin{aligned}
N\left(C_{\tilde{\tau}_{b}}^{*}\left(N_{\varphi_{b}} ; \mathbb{R}\right)\right)-N\left(C_{\tilde{\tau}_{a}}^{*}\left(N_{\varphi_{a}} ; \mathbb{R}\right)\right) & =\sum_{q \geq 0} \alpha_{b, 1}^{q} \beta_{b, 1}^{q}-\sum_{q \geq 0} \alpha_{a, 1}^{q} \beta_{a, 1}^{q} \\
& \equiv \sum_{q}\left(\alpha_{0}^{q}+\alpha_{2}^{q}\right)\left(x_{b}^{q-1}-x_{a}^{q-1}\right) \quad \bmod 2 .
\end{aligned}
$$

Combining this with (52) we obtain

$$
y_{b}^{\mathbb{R}}-y_{a}^{\mathbb{R}} \equiv \sum_{q}\left(\alpha_{0}^{q}+b_{0}^{q}+\alpha_{2}^{q}+\beta_{2}^{q}\right)\left(x_{b}^{q-1}-x_{a}^{q-1}\right) \quad \bmod 2 .
$$

In view of the fact that for a finite cochain complex of finite-dimensional vector spaces $\left(C^{*}, d^{*}\right)$ the total rank of $C^{*}$ is the same mod 2 as the total rank of its cohomology we have

$$
\alpha_{0}^{q}+b_{0}^{q} \equiv \beta_{0}^{q} \quad \bmod 2 \quad \text { and } \quad \alpha_{2}^{q}+b_{2}^{q} \equiv \beta_{2}^{q} \quad \bmod 2
$$

and with the help of (53) we find

$$
\alpha_{0}^{q}+b_{0}^{q}+\alpha_{2}^{q}+b_{2}^{q} \equiv \beta_{0}^{q}+\beta_{2}^{q} \equiv \operatorname{dim} H^{q-1}(N ; \mathbb{R}) \quad \bmod 2 .
$$

Together with (54) this implies (48), and the proof is completed.

Lemma 4.7 If $H^{*}\left(N_{\mathrm{id}} ; p^{*} \rho\right)=0$, then $\mathcal{T}_{\text {comb }}^{N_{\mathrm{id}}, \mathfrak{e}, \mathfrak{o}}\left(p^{*} \rho\right)=(-1)^{\operatorname{dim}(V) \cdot z_{\mathrm{id}}} \zeta_{\mathrm{id}}(\rho)$.

Proof Equip the interval $I$ with the three cell structure. Let $\tau_{1}$ be a smooth triangulation of $N, \tau$ the product cell structure on $N \times I$ and $\tilde{\tau}$ the induced (product) cell structure on $N_{\text {id }}=N \times S^{1}$. Then the complex $C_{\tau}^{*}\left(N \times I, N \times \partial I ; p^{*} \rho\right)$ is the suspension of $C_{\tau_{1}}^{*}\left(N ; p^{*} \rho\right)$. Using Lemma A.1 and Lemma 4.5 it is easy to see that $\mathcal{T}_{\text {comb }}^{N_{\text {id }, \mathfrak{e}, \mathfrak{o}}}\left(p^{*} \rho\right)= \pm \zeta_{\text {id }}(\rho)$.

To decide about the sign let $(-1)^{y^{\rho}}$ denote the sign in the fusion diagram of the short exact sequence

$$
0 \rightarrow C_{\tau}^{*}\left(N \times I, N \times \partial I ; p^{*} \rho\right) \rightarrow C_{\tilde{\tau}}^{*}\left(N_{\mathrm{id}} ; p^{*} \rho\right) \rightarrow C_{\tau_{1}}^{*}(N ; V) \rightarrow 0
$$

and let $(-1)^{y^{\mathbb{R}}}$ denote the sign appearing in the fusion diagram for the short exact sequence

$$
0 \rightarrow C_{\tau}^{*}(N \times I, N \times \partial I ; \mathbb{R}) \rightarrow C_{\tilde{\tau}}^{*}\left(N_{\mathrm{id}} ; \mathbb{R}\right) \rightarrow C_{\tau_{1}}^{*}(N ; \mathbb{R}) \rightarrow 0 .
$$

Similar to the proof of Lemma 4.6 the statement about the sign follows once we have shown

$$
\operatorname{dim}(V) \cdot y^{\mathbb{R}}-y^{\rho} \equiv \operatorname{dim}(V) \cdot z_{\text {id }} \quad \bmod 2 .
$$


Note that

$$
z_{\mathrm{id}}=\sum_{q}\left(\operatorname{dim} H^{q}(N ; \mathbb{R})\right)^{2} \equiv \sum_{q} \operatorname{dim} H^{q}(N ; \mathbb{R}) \equiv \chi(N) \quad \bmod 2 .
$$

Moreover let $(-1)^{y^{V}}$ denote the sign in the fusion diagram of

$$
0 \rightarrow C_{\tau}^{*}(N \times I, N \times \partial I ; V) \rightarrow C_{\tilde{\tau}}^{*}\left(N_{\mathrm{id}} ; V\right) \rightarrow C_{\tau_{1}}^{*}(N ; V) \rightarrow 0 .
$$

Note that $y^{V} \equiv \operatorname{dim}(V) y^{\mathbb{R}} \bmod 2$. In view of (56) it thus suffices to show

$$
y^{V}-y^{\rho} \equiv \operatorname{dim}(V) \chi(N) \bmod 2 .
$$

Using the acyclicity of $C_{\widetilde{\tau}_{\mathrm{id}}}^{*}\left(N_{\mathrm{id}} ; p^{*} \rho\right)$ and the simple nature of the long exact sequence associated to (57) we obtain from Lemma A.1

$$
\begin{aligned}
& y^{V}-y^{\rho}=N\left(C_{\widetilde{\tau}_{\mathrm{id}}}^{*}\left(N_{\mathrm{id}} ; V\right)\right)+\sum_{q} \operatorname{dim} H^{q-1}(N ; V) b_{2}^{q}+\sum_{q} \operatorname{dim} H^{q}(N ; V) b_{0}^{q+1} \\
& \quad=N\left(C_{\tilde{\mathrm{\tau}}_{\mathrm{id}}}^{*}\left(N_{\mathrm{id}} ; V\right)\right)+\sum_{q} \operatorname{dim} H^{q-1}(N ; V)\left(b_{2}^{q}+b_{0}^{q}\right)
\end{aligned}
$$

where $\quad b_{0}^{q}:=\operatorname{dim} B^{q}\left(C_{\tau}^{*}\left(N \times I, N \times \partial I ; p^{*} \rho\right)\right)=\operatorname{dim} B^{q}\left(C_{\tau}^{*}(N \times I, N \times \partial I ; V)\right)$

$$
b_{2}^{q}:=\operatorname{dim} B^{q}\left(C_{\tau_{1}}^{*}(N ; V)\right) .
$$

It is not hard to verify

$$
N\left(C_{\tilde{\tau}_{\mathrm{id}}}^{*}\left(N_{\mathrm{id}} ; V\right)\right) \equiv \sum_{q}\left(\alpha_{0}^{q}+\alpha_{2}^{q}\right) \operatorname{dim} H^{q-1}(N ; V) \bmod 2
$$

where $\alpha_{0}^{q}:=\sum_{j \geq q} \operatorname{dim} C_{\tau}^{j}\left(N \times I, N \times \partial I ; p^{*} \rho\right)=\sum_{j \geq q} \operatorname{dim} C_{\tau}^{j}(N \times I, N \times \partial I ; V)$

$$
\alpha_{2}^{q}:=\sum_{j \geq q} \operatorname{dim} C_{\tau_{1}}^{j}(N ; V)
$$

Let us introduce

$$
\begin{aligned}
\beta_{0}^{q}:=\sum_{j \geq q} \operatorname{dim} H^{j}\left(C_{\tau}^{*}\left(N \times I, N \times \partial I ; p^{*} \rho\right)\right) \\
=\sum_{j \geq q} \operatorname{dim} H^{j}\left(C_{\tau}^{*}(N \times I, N \times \partial I ; V)\right) \\
\beta_{2}^{q}:=\sum_{j \geq q} \operatorname{dim} H^{j}\left(C_{\tau_{1}}^{*}(N ; V)\right)
\end{aligned}
$$


and recall that $\alpha_{0}^{q}+b_{0}^{q} \equiv \beta_{0}^{q} \bmod 2$ as well as $\alpha_{2}^{q}+b_{2}^{q} \equiv \beta_{0}^{q} \bmod 2$. From (59) and (60) and the fact that $\beta_{2}^{q}+\beta_{0}^{q}=\beta_{2}^{q}+\beta_{2}^{q-1} \equiv \operatorname{dim} H^{q-1}(N ; V) \bmod 2$ we obtain

$$
\begin{aligned}
y^{V}-y^{\rho} & \equiv \sum_{q} \operatorname{dim} H^{q-1}(N ; V)\left(\beta_{2}^{q}+\beta_{0}^{q}\right) \bmod 2 \\
& \equiv \sum_{q}\left(\operatorname{dim} H^{q-1}(N ; V)\right)^{2} \quad \bmod 2 \\
& \equiv \sum_{q} \operatorname{dim} H^{q-1}(N ; V) \quad \bmod 2 \\
& \equiv \chi(N) \operatorname{dim}(V) \quad \bmod 2 .
\end{aligned}
$$

Hence we have established (58) and the proof is complete.

Proposition 4.4 follows from the previous two lemmas, and the simple observation that $H^{*}\left(N_{\varphi} ; p^{*} \rho\right)=0$ implies $H^{*}\left(N_{\text {id }} ; p^{*} \rho\right)=0$. Indeed, $H^{0}\left(N_{\varphi} ; p^{*} \rho\right)=0$ and (43) imply that $\rho(1) \in \mathrm{GL}(V)$ does not have 1 as an eigenvalue and then (43) implies that $H^{*}\left(N_{\mathrm{id}} ; p^{*} \rho\right)=0$.

Remark 4.8 Let $y \in N$ and suppose $\varphi(y)=y$. Denote by $\alpha: \pi_{1}(N, y) \rightarrow \pi_{1}(N, y)$ the isomorphism induced by $\varphi$. Let $\rho_{0}: \pi_{1}(N, y) \rightarrow \mathrm{GL}(V)$ be a representation and $A \in \mathrm{GL}(V)$ so that $\rho_{0}(\alpha(g), A(v))=A\left(\rho_{0}(g, v)\right), g \in \pi_{1}(N, y), v \in V$. Denote by $\varphi_{\tilde{\rho}}^{*}: H^{*}\left(N ; \rho_{0}\right) \rightarrow H^{*}\left(N ; \rho_{0}\right)$ resp. $\tilde{\rho}: \pi_{1}(N, y) \times_{\alpha} \mathbb{Z} \rightarrow \mathrm{GL}(V)$ the isomorphism induced by the triple $\left(\varphi, \rho_{0}, A\right)$ resp. the representation induced by the triple $\left(\alpha, \rho_{0}, A\right)$. The arguments in this section lead to a result similar to Proposition 4.4 where $H^{*}(N ; V)$ is replaced by $H^{*}\left(N ; \rho_{0}\right)$ and $H^{*}\left(M ; p^{*} \rho\right)$ by $H^{*}(M ; \tilde{\rho})$.

\subsection{An invariant with values in $\mathbb{R} / \pi \mathbb{Z}$}

If $M$ is a closed manifold with $\Gamma=\pi_{1}\left(M, x_{0}\right)$ and $\rho$ an acyclic (ie, $\left.H^{*}(M ; \rho)=0\right)$ unimodular representation then $\mathcal{T}_{\text {comb }}^{\mathfrak{e}, \mathfrak{o}}$ is by Theorem 4.3(iii) independent of $\mathfrak{e}$ and its argument mod $\pi$ independent of $\mathfrak{o}$. Denote by

$$
S(M ; \rho):=\arg \left(\mathcal{T}_{\text {comb }}^{\mathfrak{e}, \mathfrak{o}}\right) \in \mathbb{R} / \pi \mathbb{Z} .
$$

This is a topological invariant computable with the help of a triangulation. Proposition 4.4 gives a simple formula for the case $M$ is a mapping torus and $\rho$ is the pullback by $p$ of a unimodular representation of $\mathbb{Z}$, and implicitly establishes its nontriviality. In a forthcoming paper an analytic construction of this invariant will be given with the help of spectral geometry of nonpositive Laplacians. 
More generally, if $\mathfrak{e}$ is an Euler structure and $\rho_{1}, \rho_{2} \in \operatorname{Rep}_{0}^{M}(\Gamma ; V)$ then

$$
\arg \left(\mathcal{T}_{\text {comb }}^{\mathfrak{e}, \mathfrak{o}}\right)\left(\rho_{2}\right)-\arg \left(\mathcal{T}_{\text {comb }}^{\mathfrak{e}, \mathfrak{o}}\right)\left(\rho_{1}\right) \in \mathbb{R} / 2 \pi \mathbb{Z}
$$

is independent of $\mathfrak{o}$ and defines an invariant $S^{\mathfrak{e}}\left(M ; \rho_{1}, \rho_{2}\right) \in \mathbb{R} / 2 \pi \mathbb{Z}$ which can be derived from the function $\mathcal{T}_{\text {an }}^{\mathfrak{e}^{*}}, P(\mathfrak{e})=\mathfrak{e}^{*}$, and calculated with the help of any holomorphic path $\tilde{\rho}(z)$ between $\rho_{1}$ and $\rho_{2}$ as explain in the introduction,

$$
S^{\mathfrak{e}}\left(M ; \rho_{1}, \rho_{2}\right)=\Re\left(2 / \mathbf{i} \int_{1}^{2} \frac{\partial\left(\mathcal{T}_{\text {an }}^{\mathfrak{e}^{*}} \circ \widetilde{\rho}\right)}{\mathcal{T}_{\text {an }}^{\mathfrak{e}^{*}} \circ \widetilde{\rho}}\right)
$$

where $\partial \varphi$ denotes the one-form $\frac{\partial \varphi}{\partial z} d z$. As already pointed out in introduction given $\rho_{1}$ and $\rho_{2}$ two representations even in the same connected component of $\operatorname{Rep}_{0}^{M}(\Gamma ; V)$ a holomorphic path might not exist, however indirectly one can benefit from such formula.

It is interesting to compare this invariant with the one defined using the spectral flow in [1] for an odd-dimensional manifold and a unitary representation [14]. More will be said in the forthcoming paper; see also the recent preprints $[6 ; 4 ; 5 ; 3]$.

\subsection{Marcsik's theorem}

In this section we will use the Bismut-Zhang theorem and Proposition 4.4 to give a proof of a theorem due to J Marcsik. We continue to use the notation from Section 4.3.

Let $g$ be a Riemannian metric on $M$ and $\omega$ a real-valued closed one-form on $M$. For $z \in \mathbb{C}$ denote by $d_{\omega}(z) \alpha:=d \alpha+z \omega \wedge \alpha$ the Witten deformed differential on $\Omega^{*}(M ; \mathbb{C})$ and by $\delta_{\omega}(z)$ its adjoint. Denote by

$$
\Delta_{\omega}(z):=d_{\omega}(z) \delta_{\omega}(z)+\delta_{\omega}(z) d_{\omega}(z)
$$

the Laplacian, and by $\Delta_{\omega}^{k}(z)$ the Laplacian on $k$ forms. Introduce the Ray-Singer torsion

$$
T_{\text {an }}(\omega, g)(z):=\exp \left(\frac{1}{2} \sum_{k}(-1)^{k+1} k \log \operatorname{det}^{\prime} \Delta_{\omega}^{k}(z)\right) .
$$

Here $\operatorname{det}^{\prime} \Delta_{\omega}^{k}(z)$ denotes the regularized determinant of the nonnegative self-adjoint elliptic operator $\Delta_{\omega}^{k}(z)$ obtained by ignoring the zero modes.

Identifying $\mathbb{C}^{*}=\mathrm{GL}(\mathbb{C})$ we have $P_{\varphi}^{k}(w)$ and $\zeta_{\varphi}(w)$ defined for all $w \in \mathbb{C}^{*}$; see Section 4.3.

Theorem 4.9 (Marcsik [22]) Let $N$ be a closed manifold, $\varphi: N \rightarrow N$ a diffeomorphism, $M$ the mapping torus of $\varphi, p: M \rightarrow S^{1}$ the canonical projection, and $\omega$ the 
pullback of the canonical volume form on $S^{1}$. Let $g$ be a Riemannian metric on $M$, and suppose $X$ is a vector field on $M$ with $\omega(X)<0$. Then

$$
\log T_{\mathrm{an}}(\omega, g)(z)=\log \left|\zeta_{\varphi}\left(e^{z}\right)\right|+\Re(z) \int_{M} \omega \wedge X^{*} \Psi(g)
$$

for all $z \in \mathbb{C}$ which satisfy $P_{\varphi}^{k}\left(e^{z}\right) \neq 0$ for all $k .^{12}$

Proof Consider the trivial line bundle $M \times \mathbb{C}$ over $M$. Let $\nabla^{0}$ denote the trivial flat connection, and let $\mu$ denote the standard Hermitian structure on $M \times \mathbb{C}$. For $z \in \mathbb{C}$ define a connection $\nabla^{z}:=\nabla+z \omega$ on $M \times \mathbb{C}$. This connection is flat for $\omega$ is closed. Clearly $d^{\nabla^{z}}=d_{\omega}(z)$, and hence

$$
T_{\text {an }}(\omega, g)(z)=T_{\text {an }}\left(\nabla^{z}, g, \mu\right) .
$$

Define a co-Euler structure $\mathfrak{e}^{*}:=\left[g, X^{*} \Psi(g)\right] \in \mathfrak{E u l}^{*}(M ; \mathbb{R})$. Recall the Euler structure $\mathfrak{e}=[X, 0] \in \mathfrak{E u l}(M ; \mathbb{Z})$. From Theorem 3.10(iv) we see that $\mathbb{T}\left(\mathfrak{e}, \mathfrak{e}^{*}\right)=0$. Assume $P_{\varphi}^{k}\left(e^{z}\right) \neq 0$ for all $k$. Then the Bismut-Zhang theorem implies

$$
\left|\mathcal{T}_{\text {comb }}^{\mathfrak{e}, \mathfrak{o}}\left(e^{z}\right)\right|=T_{\text {an }}\left(\nabla^{z}, g, \mu\right) \cdot e^{-S\left(\omega\left(\nabla^{z}, \mu\right), X^{*} \Psi(g)\right)} .
$$

See Remark 3.18. Note that $\nabla^{z} \mu=-2 \Re(z \omega) \mu$, hence $\omega\left(\nabla^{z}, \mu\right)=\Re(z) \omega$, and thus

$$
S\left(\omega\left(\nabla^{z}, \mu\right), X^{*} \Psi(g)\right)=\mathfrak{i}(z) \int_{M} \omega \wedge X^{*} \Psi(g) .
$$

From Proposition 4.4 we get $\mathcal{T}_{\text {comb }}^{\mathfrak{e}, \mathfrak{o}}\left(e^{z}\right)= \pm \zeta_{\varphi}\left(e^{z}\right)$. Putting everything together and taking the logarithm completes the proof.

\subsection{An application}

The concept of holomorphic (or $\mathbb{C}$-differentiable) maps between open sets of complex Banach/Fréchet spaces of possibly infinite dimension is the same as in finite dimension and so are the concepts of meromorphic map, complex analytic set, complex analytic manifold $[13 ; 27 ; 12 ; 19 ; 21]$. The reader unfamiliar with the infinite-dimensional holomorphy should be aware that:

(i) If $U \subseteq \mathbb{C}$ is open and $E$ is a Fréchet space, then all possible definitions for holomorphic curves $U \rightarrow E$ yield the same concept; see for instance Kriegl and Michor [21, Theorem 7.4]. A curve $c: U \rightarrow E$ is holomorphic if and only if $\partial c / \partial \bar{z}=0$ and this is the case if and only if $\varphi \circ c: U \rightarrow \mathbb{C}$ is holomorphic for every continuous linear functional $\varphi \in E^{*}$.

\footnotetext{
${ }^{12}$ This statement is slightly more precise than the one formulated in [22].
} 
(ii) If $V \subseteq F$ is an open subset of a Fréchet space, and $E$ is another Fréchet space, then a map $f: V \rightarrow E$ is holomorphic if and only if it maps holomorphic curves in $V$ to holomorphic curves in $E$. In view of (i) a mapping $f: V \rightarrow E$ is holomorphic if and only if for every continuous linear functional $\varphi \in E^{*}$ and every holomorphic curve $c: U \rightarrow V$ the composition $\varphi \circ f \circ c$ is holomorphic. This is the case if and only if, $f$ is continuous and holomorphic along affine complex lines in $F$; see [21, Theorem 7.19], [19, Chapter III sections 2 and 3] or [12, pages 57, 58].

(iii) A subset $X \subseteq E$ of a Fréchet space is called complex analytic subset if for every point $x \in X$ there exists an open neighborhood $U_{x}$ of $x$ in $E$, a Fréchet space $F_{x}$ and a holomorphic map $f_{x}: U_{x} \rightarrow F_{x}$ such that $X \cap U_{x}=f_{x}^{-1}(0)$ [12, page 383].

(iv) Suppose $X$ is a complex analytic subset of a Fréchet space $E$ and $Y$ is a complex analytic subset of a Fréchet space $F$. A map $f: X \rightarrow Y$ is called holomorphic if for every point $x \in X$ there exists an open neighborhood $U_{x}$ of $x$ in $E$ and a holomorphic function $f_{x}: U_{x} \rightarrow F$ whose restriction to $X$ coincides with $f$.

(v) Suppose $X$ is a complex analytic subset of a Fréchet space $E . X$ is called locally irreducible if for any $x \in X$ the local ring of germs of holomorphic functions $\mathfrak{o}_{x}(X)$ is an integral domain. This concept is used mostly for finite-dimensional complex analytic sets.

If $S \subseteq X$ is a complex analytic subset of $X$ it is called proper for $X$, if the closure of $X \backslash S$ is dense in $X$.

(vi) If $X$ is a finite-dimensional irreducible complex analytic set of a Fréchet space $E$ and $S \subseteq X$ a proper analytic subset, a meromorphic function on $X$ with poles in $S$ is a function $f: X \backslash S \rightarrow \mathbb{C}$ s.t. for any $x \in X$ there exists a neighborhood $U_{x}$ and two holomorphic functions $h_{x}: U_{x} \rightarrow \mathbb{C}$ and $g_{x}: U_{x} \rightarrow \mathbb{C}, g_{x} \neq 0$ on $U_{x} \backslash S$, so that $\left.f\right|_{U_{x} \backslash S}=\left.\left(h_{x} / g_{x}\right)\right|_{U_{x} \backslash S}$.

If $X$ is a complex analytic subset of a Fréchet space $E$ and $S \subseteq X$ a proper analytic subset, a meromorphic function on $X$ with poles in $S$ is a function $f: X \backslash S \rightarrow \mathbb{C}$ which, when restricted to any finite-dimensional locally irreducible analytic subset $V \subseteq X$ with $V \cap S$ proper in $V$, is meromorphic with poles in $V \cap S$.

The above definitions can be formulated in the case where $E$ is replaced by a complex analytic Frechét manifold.

Suppose that $p: F \rightarrow M$ is a smooth complex vector bundle which admits flat connections. Denote by $\mathcal{C}(F)$ the space of all connections on $F$. This is an affine Fréchet space over $\mathbb{C}$ when equipped with the $C^{\infty}$-topology. In view of (ii) above the map which associates to each connection its curvature is a holomorphic map from the 
affine Fréchet space $\mathcal{C}(F)$ to the Fréchet space $\Omega^{2}(M ; \operatorname{End}(F))$. Hence the set of flat connections $\mathcal{F C}(F)$ is a closed complex analytic subset of $\mathcal{C}(F)$.

Let $x_{0} \in M$ be a base point, let $\Gamma:=\pi_{1}\left(M, x_{0}\right)$ denote the fundamental group and let $V:=F_{x_{0}}$ denote the fiber over $x_{0}$. The holonomy map $\mathcal{F C}(F) \rightarrow \operatorname{Rep}(\Gamma ; V)$ which associates to a flat connection $\nabla$ its holonomy representation $\rho_{\nabla}$, is a holomorphic map between complex analytic sets. To see this we first choose $r$ smooth paths $\gamma_{i}:[0,1] \rightarrow$ $M, i=1, \ldots, r$, with $\gamma_{i}(0)=\gamma_{i}(1)=x_{0}$, which represent a collection of generators of $\Gamma=\pi_{1}\left(M, x_{0}\right)$. For a connection $\nabla$ denote the isomorphisms defined by parallel transport along $\gamma_{i}$ by $P_{i}^{\nabla} \in \operatorname{End}(V), V=F_{x_{0}}$. Define $\pi: \mathcal{C}(F) \rightarrow \operatorname{End}(V)^{r} \times \mathbb{C}$ by

$$
\pi(\nabla):=\left(P_{1}^{\nabla}, \ldots, P_{r}^{\nabla}, \operatorname{det}\left(P_{1}^{\nabla}\right)^{-1} \cdots \operatorname{det}\left(P_{r}^{\nabla}\right)^{-1}\right) .
$$

This is a holomorphic map in view of (ii) above. Since its restriction to $\mathcal{F C}(F)$ coincides with the holonomy map $\mathcal{F C}(F) \rightarrow \operatorname{Rep}(\Gamma ; V)$, the latter is holomorphic.

Denote by $\Sigma(M)$ the set of representations $\rho \in \operatorname{Rep}(\Gamma ; V)$ which are not local minima for the function $d(\rho):=\sum_{i=0}^{n} \operatorname{dim} H^{i}(M ; \rho)$. Observe that this is an algebraic subset of $\operatorname{Rep}(\Gamma ; V)$. In particular it is a closed analytic subset of $\operatorname{End}(V)^{r} \times \mathbb{C}$. Let $\Sigma(F)$ denote the subset of flat connections $\nabla$ which are not a local minimum for the function $d: \mathcal{F C}(F) \rightarrow \mathbb{Z}$ defined by $d(\nabla):=\sum_{i=0}^{n} \operatorname{dim} H^{i}\left(M ; \rho_{\nabla}\right)$. Since $\Sigma(F)$ is the intersection $\mathcal{F C}(F) \cap \pi^{-1}(\Sigma(M))$, the set $\Sigma(F)$ is a closed analytic subset of $\mathcal{F C}(F)$.

Denote by $\mathcal{F C}^{M}(F)$ the flat connections whose holonomy representations are in $\operatorname{Rep}^{M}(\Gamma ; V)$. Observe that $\mathcal{F C}^{M}(F)$ is a closed analytic subset of $\mathcal{F C}(F)$.

Let $g$ be a Riemannian metric on $M$ and let $\mu$ be a Hermitian fiber metric on $F$. The Ray-Singer torsion $T_{\text {an }}(\nabla, g, \mu)$ defines a positive real-valued function

$$
T_{\mathrm{an}}^{g, \mu}: \mathcal{F C}(F) \rightarrow \mathbb{R}
$$

The following is a straightforward consequence of Theorem 4.3 and Proposition 2.2.

Corollary 4.10 Let $M$ be a closed connected manifold, let $F$ be a complex vector bundle over $M$, let $g$ be a Riemannian metric on $M$ and let $\mu$ be a Hermitian fiber metric on $F$. Then there exists a meromorphic function on $\mathcal{F C}^{M}(F)$ whose zeros and poles are contained in $\Sigma(F)$ and whose restriction to $\mathcal{F C}^{M}(F) \backslash \Sigma(F)$ has $T_{\text {an }}^{g, \mu}$ as absolute value.

Proof Choose a base point $x_{0} \in M$ and an Euler structure $\mathfrak{e} \in \mathfrak{E u l}_{x_{0}}(M ; \mathbb{Z})$. Choose a homology orientation $\mathfrak{o}$ of $M$. Let $\Gamma=\pi_{1}\left(M, x_{0}\right)$ denote the fundamental group, and let $V=F_{x_{0}}$ denote the fiber of $F$ over $x_{0}$. In view of Theorem 4.3(i) $\mathcal{T}_{\text {comb }}^{\mathfrak{e}, \mathfrak{o}}$ is 
a rational function on $\operatorname{Rep}^{M}(\Gamma ; V)$ with zeros and poles contained in $\Sigma(M)$. Since the holonomy map $\pi: \mathcal{F C}^{M}(F) \rightarrow \operatorname{Rep}^{M}(\Gamma ; V)$ is holomorphic, the composition $f_{1}:=\mathcal{T}_{\text {comb }}^{\mathfrak{e}, \mathfrak{o}} \circ \pi$ is thus a meromorphic function on $\mathcal{F C}^{M}(F)$ with zeros and poles contained in $\Sigma(F)$. Consider the co-Euler structure $\mathfrak{e}^{*}:=P(\mathfrak{e})$ and choose $\alpha$ such that $\mathfrak{e}^{*}=[g, \alpha]$. Let $\widetilde{\omega}: \mathcal{C}(F) \rightarrow \Omega^{1}(M ; \mathbb{C})$ be an affine map as in Proposition 2.2. Its restriction to $\mathcal{F C}(F)$ is certainly holomorphic, and thus the map $f_{2}: \mathcal{F C}(F) \rightarrow \mathbb{C}^{*}$, $f_{2}(\nabla):=e^{S(\widetilde{\omega}(\nabla, \mu), \alpha)}$ is holomorphic too. The product $f:=f_{1} \cdot f_{2}$ therefore is a meromorphic function on $\mathcal{F C}^{M}(F)$ with zeros and poles are contained $\Sigma(F)$. In view of Theorem 4.3(ii) we have

$$
|f(\nabla)|=\left|f_{1}(\nabla)\right| e^{\Re(S(\widetilde{\omega}(\nabla, \mu), \alpha))}=\left|\mathcal{T}_{\text {comb }}^{\mathfrak{e}, \mathfrak{o}}\left(\rho_{\nabla}\right)\right| e^{S(\Re(\widetilde{\omega}(\nabla, \mu)), \alpha)}=\left|T_{\mathrm{an}}^{g, \mu}(\nabla)\right|
$$

for all $\nabla \in \mathcal{F C}^{M}(F) \backslash \Sigma(F)$.

If the dimension of $M$ is odd, the Ray-Singer torsion defines a positive real-valued function

$$
T_{\text {an }}: \operatorname{Rep}^{M}(\Gamma ; V) \backslash \Sigma(M) \rightarrow \mathbb{R}, \quad T_{\text {an }}(\rho):=T_{\text {an }}\left(\nabla_{\rho}, g, \mu\right)
$$

where $g$ is any Riemannian metric on $M$, and $\mu$ is any fiber metric in $F_{\rho}$. In view of the Hermitian anomaly [2, Theorem 0.1 ] and because the Euler form $E(g)$ is identically zero, this is indeed independent of $\mu$ and $g$.

Corollary 4.11 Let $M$ be an odd-dimensional closed connected manifold, let $\Gamma=$ $\pi_{1}\left(M, x_{0}\right)$ denote its fundamental group and let $V$ be a finite-dimensional complex vector space. Then there exists a rational function on $\operatorname{Rep}^{M}(\Gamma ; V)$ whose restriction to $\operatorname{Rep}^{M}(\Gamma ; V) \backslash \Sigma(M)$ has $\left(T_{\mathrm{an}}\right)^{2}$ as absolute value.

If the involution $v$ on $\mathfrak{E u l}(M ; \mathbb{Z})$ has a fixed point, or more generally, if the canonical Euler structure $\mathfrak{e}_{\text {can }} \in \mathfrak{E u r}(M ; \mathbb{R})$ is integral (Remark 3.2) then even $T_{\text {an }}$ is the absolute value of a rational function on $\operatorname{Rep}^{M}(\Gamma ; V)$.

Proof Choose an Euler structure $\mathfrak{e} \in \mathfrak{E u t}(M ; \mathbb{Z})$ and a homology orientation $\mathfrak{o}$ of $M$. From Theorem 4.3(i) we know that $h_{1}:=\left(\mathcal{T}_{\text {comb }}^{\mathfrak{e}, \mathfrak{o}}\right)^{2}$ is a rational function on $\operatorname{Rep}^{M}(\Gamma ; V)$. We want to construct an additional regular function $h_{2}: \operatorname{Rep}(\Gamma ; V) \rightarrow \mathbb{C}$ so that the product $h_{1} \cdot h_{2}$ has as absolute value $\left(T_{\mathrm{an}}\right)^{2}$.

Choose a Riemannian metric $g$ on $M$. Consider the co-Euler structure $\mathfrak{e}^{*}:=P(\mathfrak{e})$, and choose $\alpha \in \Omega^{n-1}\left(M ; \mathcal{O}_{M}\right)$ such that $\mathfrak{e}^{*}=[g, \alpha]$. Note that $d \alpha=E(g)=0$, hence $\alpha$ defines a cohomology class $[\alpha] \in H^{n-1}\left(M ; \mathcal{O}_{M}\right)$. We claim that the preimage $\operatorname{PD}^{-1}([2 \alpha]) \in H_{1}(M ; \mathbb{R})$ is integral. We postpone this first step to the second half of this proof. 
Next, we choose closed loops $\gamma_{i}$ based at $x_{0}$ in $M, 1 \leq i \leq r$, which form a set of generators of $\Gamma$. Since $\mathrm{PD}^{-1}([2 \alpha])$ is integral we find integers $m_{i} \in \mathbb{Z}$ such that $\mathrm{PD}^{-1}([2 \alpha])=\sum_{i=1}^{r} m_{i} \gamma_{i}$ in $H_{1}(M ; \mathbb{R})$. We then have

$$
\sum_{i=1}^{r} m_{i} \int_{\gamma_{i}} \omega=2 \int_{M} \omega \wedge \alpha
$$

for every closed 1 -form $\omega \in \Omega^{1}(M ; \mathbb{R})$. Consider the regular map

$$
h_{2}: \operatorname{Rep}(\Gamma ; V) \rightarrow \mathbb{C}, \quad h_{2}(\rho):=\prod_{i=1}^{r}\left(\operatorname{det}\left(\rho\left(\gamma_{i}\right)\right)\right)^{m_{i}}
$$

The product $h:=h_{1} \cdot h_{2}$ is a rational function on $\operatorname{Rep}^{M}(\Gamma ; V)$. We claim that $T_{\text {an }}(\rho)^{2}=|h(\rho)|$ for all $\rho \in \operatorname{Rep}^{M}(\Gamma ; V) \backslash \Sigma(M)$.

To see this, fix $\rho \in \operatorname{Rep}^{M}(\Gamma ; V) \backslash \Sigma(M)$, consider the vector bundle $F_{\rho}$ over $M$ with flat connection $\nabla_{\rho}$ and choose a Hermitian fiber metric $\mu$ on $F_{\rho}$. Recall from Remark 2.1 that

$$
\left|\operatorname{det}\left(\rho\left(\gamma_{i}\right)\right)\right|=\exp \int_{\gamma_{i}} \omega\left(\nabla_{\rho}, \mu\right)
$$

for all $1 \leq i \leq r$. We conclude

$$
\begin{aligned}
\left|h_{2}(\rho)\right| & =\prod_{i=1}^{r}\left|\operatorname{det}\left(\rho\left(\gamma_{i}\right)\right)\right|^{m_{i}}=\prod_{i=1}^{r}\left(\exp \int_{\gamma_{i}} \omega\left(\nabla_{\rho}, \mu\right)\right)^{m_{i}} \\
& =\exp \left(\sum_{i=1}^{r} m_{i} \int_{\gamma_{i}} \omega\left(\nabla_{\rho}, \mu\right)\right)=\exp \left(2 \int_{M} \omega\left(\nabla_{\rho}, \mu\right) \wedge \alpha\right)=e^{2 S\left(\omega\left(\nabla_{\rho}, \mu\right), \alpha\right)}
\end{aligned}
$$

Together with Theorem 4.3(ii) we obtain

$$
|h(\rho)|=\left|h_{1}(\rho)\right|\left|h_{2}(\rho)\right|=\left|\mathcal{T}_{\text {comb }}^{\mathfrak{e}, \mathfrak{o}}(\rho)\right|^{2} e^{2 S\left(\omega\left(\nabla_{\rho}, \mu\right), \alpha\right)}=T_{\text {an }}\left(\nabla_{\rho}, g, \mu\right)^{2}=T_{\text {an }}(\rho)^{2} .
$$

Thus $\left(T_{\mathrm{an}}\right)^{2}$ is indeed the absolute value of the rational map $h$.

Next we show that $\operatorname{PD}^{-1}([2 \alpha]) \in H_{1}(M ; \mathbb{R})$ is an integral class. Recall from Remark 3.2 that there is a canonical Euler structure $\mathfrak{e}_{\text {can }} \in \mathfrak{E} \mathfrak{E} l(M ; \mathbb{R})$ which is the unique fixed point of the involution $v$ on $\mathfrak{E u l}(M ; \mathbb{R})$. Note that $\mathfrak{e}_{\text {can }}$ will in general not be an integral Euler structure. We identify $H_{1}(M ; \mathbb{R})$ with $\mathfrak{E} u \mathfrak{l}(M ; \mathbb{R})$ by the map $a \mapsto \mathfrak{e}_{\text {can }}+a$, and denote this identification by $\mathfrak{e}_{\text {can }}+\bullet: H_{1}(M ; \mathbb{R}) \rightarrow \mathfrak{E u l}(M ; \mathbb{R})$.

Similarly we use the canonical co-Euler structure $\mathfrak{e}_{\text {can }}^{*}=[g, 0] \in \mathfrak{E} \mathfrak{l}^{*}(M ; \mathbb{R})$ of Remark 3.8 to identify $H^{n-1}\left(M ; \mathcal{O}_{M}\right)$ with $\mathfrak{E u l}^{*}(M ; \mathbb{R})$ by the map $[\beta] \mapsto \mathfrak{e}_{\text {can }}^{*}+[\beta]=[g,-\beta]$, and denote this identification by $\mathfrak{e}_{\text {can }}^{*}+\bullet: H^{n-1}\left(M ; \mathcal{O}_{M}\right) \rightarrow \mathfrak{E u l}^{*}(M ; \mathbb{R})$. 
Since from Proposition 3.9, $P: \mathfrak{E u l}(M ; \mathbb{R}) \rightarrow \mathfrak{E u l}^{*}(M ; \mathbb{R})$ intertwines the canonical involutions on $\mathfrak{E u l}(M ; \mathbb{R})$ and $\mathfrak{E u l}^{*}(M ; \mathbb{R})$ we must have $P\left(\mathfrak{e}_{\text {can }}\right)=\mathfrak{e}_{\text {can }}^{*}$.

We also use the Euler structure $\mathfrak{e}$ to identify $H_{1}(M ; \mathbb{Z})$ with $\mathfrak{E} u l(M ; \mathbb{Z})$ by the map $c \mapsto \mathfrak{e}+c$, and denote this identification by $\mathfrak{e}+\bullet: H_{1}(M ; \mathbb{Z}) \rightarrow \mathfrak{E u l}(M ; \mathbb{Z})$.

Then there exists $a_{0} \in H_{1}(M ; \mathbb{R})$ so that the canonical map $\mathfrak{E u l}(M ; \mathbb{Z}) \rightarrow \mathfrak{E u l}(M ; \mathbb{R})$ becomes, via the above identifications, the map $c \mapsto a_{0}+c$.

We summarize these observations in the following commutative diagram:

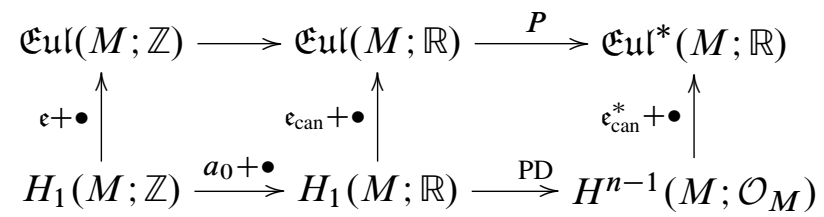

The commutativity of the diagram gives $\mathrm{PD}^{-1}([2 \alpha])=-2 a_{0}$.

To see that $2 a_{0}$ is actually an integral homology class we note that via the identification $\mathfrak{e}_{\text {can }}+\bullet$ the canonical involution on Euler structures with real coefficients is given by $v(a)=-a, a \in H_{1}(M ; \mathbb{R})$. Via the identification $\mathfrak{e}+\bullet$ the canonical involution on Euler structures with integer coefficients must be of the form $v(c)=c_{0}-c$ for some $c_{0} \in H_{1}(M ; \mathbb{Z})$. Since the canonical map $\mathfrak{E u l}(M ; \mathbb{Z}) \rightarrow \mathfrak{E u l}(M ; \mathbb{R})$ intertwines these canonical involutions, we must have $a_{0}+c_{0}=-a_{0}$. Hence $\mathrm{PD}^{-1}([2 \alpha])=-2 a_{0}=c_{0}$ is indeed integral.

For the last statement of the proposition, note first that any fixed point of the involution on $\mathfrak{E} u l(M ; \mathbb{Z})$ must be mapped to $\mathfrak{e}_{\text {can }}$ via the canonical map from $\mathfrak{E} \mathfrak{u l}(M ; \mathbb{Z})$ to $\mathfrak{E} \mathfrak{u l}(M ; \mathbb{R})$. If the involution on $\mathfrak{E u l}(M ; \mathbb{Z})$ admits a fixed point then the Euler structure $\mathfrak{e}_{\text {can }}$ must therefore be integral. If $\mathfrak{e}_{\text {can }}$ is integral then $\operatorname{PD}^{-1}([\alpha])=-a_{0}$ must be integral. Proceeding as above, one constructs in this case a rational function on $\operatorname{Rep}^{M}(\Gamma ; V)$ with absolute value $T_{\text {an }}$.

Example 4.12 Consider the circle $M=S^{1}$ and the one-dimensional vector space $V=\mathbb{C}$. In this case $\Gamma=\mathbb{Z}, \operatorname{Rep}(\Gamma ; V)=\mathbb{C} \backslash\{0\}, \operatorname{Rep}_{0}^{M}(\Gamma ; V)=\mathbb{C} \backslash\{0,1\}$ and $\operatorname{Rep}^{M}(\Gamma ; V)=\mathbb{C} \backslash\{0\}$. From Theorem 4.9, or via a direct computation, we see that in this case the Ray-Singer torsion is given by

$$
T_{\text {an }}: \operatorname{Rep}^{M}(\Gamma ; V)=\mathbb{C} \backslash\{0\} \rightarrow \mathbb{C}, \quad T_{\text {an }}(z)=\frac{|z-1|}{\sqrt{|z|}} .
$$

Therefore the Ray-Singer torsion will in general not be the absolute value of a rational function on $\operatorname{Rep}^{M}(\Gamma ; V)$. The square of the Ray-Singer torsion, however, is the 
absolute value of the rational function $z \mapsto(z-1)^{2} / z$ which happens to have a zero at $\Sigma(M)=\{1\}$.

\section{Appendix A Some homological algebra}

Let $\mathbb{K}$ be a field of characteristics zero. Suppose $C^{*}$ is a finite-dimensional $\mathbb{Z}$-graded complex over $\mathbb{K}$ with differential of degree one, $d^{q}: C^{q} \rightarrow C^{q+1}$. We will always assume $C^{q}=0$ for $q<0$. Let us write $Z^{q}:=\operatorname{ker} d^{q} \subseteq C^{q}$ for the cocycles, $B^{q}:=\operatorname{img} d^{q-1} \subseteq C^{q}$ for the coboundaries, and $H^{q}:=Z^{q} / B^{q}$ for the cohomology.

Let $\mathfrak{c}^{q}$ be a base of $C^{q}$, let $\mathfrak{h}^{q}$ be a base of $H^{q}$, and let $\mathfrak{b}^{q}$ be a base of $B^{q}$. Recall the short exact sequences

$$
0 \rightarrow Z^{q} \rightarrow C^{q} \stackrel{d}{\rightarrow} B^{q+1} \rightarrow 0 \quad \text { and } \quad 0 \rightarrow B^{q} \rightarrow Z^{q} \rightarrow H^{q} \rightarrow 0 .
$$

Choose lifts $\widetilde{\mathfrak{h}}^{q}$ in $Z^{q}$ of $\mathfrak{h}^{q}$. Then $\mathfrak{b}^{q} \widetilde{\mathfrak{h}}^{q}$ is a base of $Z^{q}$. Choose lifts $\widetilde{\mathfrak{b}}^{q+1}$ in $C^{q}$ of $\mathfrak{b}^{q+1}$. Then $\left(\mathfrak{b}^{q} \tilde{\mathfrak{h}}^{q}\right) \tilde{\mathfrak{b}}^{q+1}$ is a base of $C^{q}$. For two bases $\mathfrak{a}_{1}$ and $\mathfrak{a}_{2}$ of a common vector space we write $\left[\mathfrak{a}_{1} / \mathfrak{a}_{2}\right]$ for the determinant of the matrix expressing $\mathfrak{a}_{1}$ in terms of $\mathfrak{a}_{2}$. The quantity $\prod_{q}\left[\mathfrak{c}^{q} /\left(\mathfrak{b}^{q} \tilde{\mathfrak{h}}^{q}\right) \tilde{\mathfrak{b}}^{q+1}\right]^{(-1)^{q}}$ does not depend on the choice of $\mathfrak{b}^{q}$ and does not depend on the choice of lifts $\tilde{\mathfrak{b}}^{q}$ and $\tilde{\mathfrak{h}}^{q}$ [24].

Recall that the determinant line of a finite-dimensional vector space $V$ is defined as $\operatorname{det} V:=\Lambda^{\operatorname{dim} V} V$. Moreover, we will write $L^{1}:=L$ and $L^{-1}:=\operatorname{Hom}(L ; \mathbb{K})$ for a line, ie, one-dimensional vector space, $L$. Consider the graded determinant line of $C^{*}$

$$
\operatorname{det} C^{*}:=\bigotimes_{q}\left(\operatorname{det} C^{q}\right)^{(-1)^{q}}
$$

and let $\mathfrak{c}$ denote the base of $\operatorname{det} C^{*}$ induced from the bases $\mathfrak{c}^{q}$. Similarly, let $\mathfrak{h}$ denote the base of det $H^{*}$ induced from the bases $\mathfrak{h}^{q}$. Consider the isomorphism

$$
\varphi_{C^{*}}: \operatorname{det} C^{*} \rightarrow \operatorname{det} H^{*}, \quad \mathfrak{c} \mapsto(-1)^{N} \prod_{q}\left[\mathfrak{c}^{q} /\left(\mathfrak{b}^{q} \tilde{\mathfrak{h}}^{q}\right) \tilde{\mathfrak{b}}^{q+1}\right]^{(-1)^{q}} \mathfrak{h}
$$

where $N:=\sum_{q \geq 0} \alpha^{q} \beta^{q}$ with $\alpha^{q}:=\sum_{j \geq q} \operatorname{dim} C^{j}$ and $\beta^{q}:=\sum_{j \geq q} \operatorname{dim} H^{j}$ [15; 32]. The isomorphism $\varphi_{C^{*}}: \operatorname{det} C^{*} \rightarrow \operatorname{det} H^{*}$ does not depend on the choice of bases $\mathfrak{c}^{q}$ or $\mathfrak{h}^{q}$. The sign $(-1)^{N}$ is referred to as Turaev's sign refinement. Note that $(-1)^{N}=1$ if $C^{*}$ is acyclic.

Suppose we have a short exact sequence of finite-dimensional $\mathbb{Z}$-graded cochain complexes over $\mathbb{K}$ :

$$
0 \rightarrow C_{0}^{*} \rightarrow C_{1}^{*} \rightarrow C_{2}^{*} \rightarrow 0
$$


Then there are isomorphisms $\varphi_{C_{i}^{*}}: \operatorname{det} C_{i}^{*} \rightarrow \operatorname{det} H_{i}^{*}, i=0,1,2$. Let $\mathcal{H}^{*}$ denote the corresponding long exact sequence

(64) $0 \rightarrow H_{0}^{0} \rightarrow H_{1}^{0} \rightarrow H_{2}^{0} \stackrel{\delta^{0}}{\rightarrow} H_{0}^{1} \rightarrow \cdots \stackrel{\delta^{q-1}}{\rightarrow} H_{0}^{q} \rightarrow H_{1}^{q} \rightarrow H_{2}^{q} \stackrel{\delta^{q}}{\rightarrow} H_{0}^{q+1} \rightarrow \cdots$

considered as (acyclic) $\mathbb{Z}$-graded cochain complex, the grading so that $\mathcal{H}^{3 q+i}=H_{i}^{q}$. As an acyclic complex it provides an isomorphism $\varphi_{\mathcal{H}^{*}}: \operatorname{det} \mathcal{H}^{*} \rightarrow \mathbb{K}$ which we regard as an isomorphism $\varphi_{\mathcal{H}^{*}}$ : $\operatorname{det} H_{0}^{*} \otimes \operatorname{det} H_{2}^{*} \rightarrow \operatorname{det} H_{1}^{*}$.

The short exact sequence (63) provides isomorphisms $\operatorname{det} C_{0}^{q} \otimes \operatorname{det} C_{2}^{q}=\operatorname{det} C_{1}^{q}$ for all $q$, and then an isomorphism $\psi: \operatorname{det} C_{0}^{*} \otimes \operatorname{det} C_{2}^{*} \rightarrow \operatorname{det} C_{1}^{*}$. For a proof of the following lemma consult Nicolaescu [25, Proposition 1.18] or look carefully at the proof of Theorem 3.2 in Milnor [24].

Lemma A.1 The following diagram commutes:

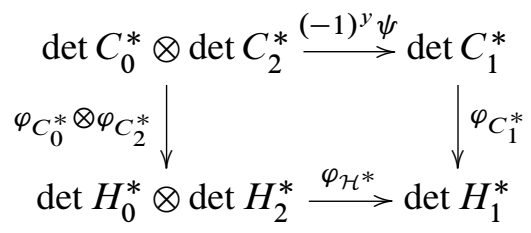

Here $y=N\left(C_{0}^{*}\right)+N\left(C_{1}^{*}\right)+N\left(C_{2}^{*}\right)+\sum_{q} f_{1}^{q} \cdot b_{2}^{q}+b_{2}^{q} \cdot b_{0}^{q+1}+f_{2}^{q} \cdot b_{0}^{q+1}$ with $b_{i}^{q}:=\operatorname{dim} B^{q}\left(C_{i}^{*}\right)$ and $f_{i}^{q}:=\operatorname{dim} B^{3 q+i}\left(\mathcal{H}^{*}\right)$.

Following Farber and Turaev [15] we call the above diagram the fusion diagram, and $(-1)^{y} \psi$ the fusion isomorphism. Note however, that the fusion homomorphism in general depends on the differentials of the complexes. If the short exact sequence of complexes splits it does not [15].

Lemma A.2 In the situation of Lemma $A .1$ suppose in addition that $C_{2}^{*}$ is acyclic and two-dimensional, concentrated in degree $k$ and $k+1$. Then the sign $(-1)^{y}$ does not depend on the differentials on $C_{0}^{*}$ and $C_{1}^{*}$.

Proof Indeed we have

$$
\begin{aligned}
N\left(C_{0}^{*}\right)+N\left(C_{1}^{*}\right) & =\beta^{k+1}\left(C_{0}^{*}\right) \quad \bmod 2 \\
N\left(C_{2}^{*}\right) & =0 \\
f_{1}^{q} & =\operatorname{dim} H_{1}^{q}=\operatorname{dim} H_{0}^{q} \\
f_{2}^{q} & =0 \\
b_{2}^{q} & =0 \quad \text { for } q \neq k+1 \\
b_{2}^{k+1} & =1 .
\end{aligned}
$$


Using the general fact $\alpha^{q}\left(C_{0}^{*}\right)+\beta^{q}\left(C_{0}^{*}\right)+b_{0}^{q}=0 \bmod 2$ this implies $y=\beta^{k+1}\left(C_{0}^{*}\right)+\operatorname{dim} H_{0}^{k+1}+b_{0}^{k+2}=\beta^{k+2}\left(C_{0}^{*}\right)+b_{0}^{k+2}=\alpha^{k+2}\left(C_{0}^{*}\right) \bmod 2$.

Hence $(-)^{y}$ does not depend on the differentials.

Since elementary expansions give rise to short exact sequences as in Lemma A.2, we have the following corollary.

Corollary A.3 The Milnor-Turaev torsion (Definition 3.12) does indeed not depend on the triangulation.

\section{References}

[1] M F Atiyah, V K Patodi, I M Singer, Spectral asymmetry and Riemannian geometry. II, Math. Proc. Cambridge Philos. Soc. 78 (1975) 405-432 MR0397798

[2] J-M Bismut, W Zhang, An extension of a theorem by Cheeger and Müller, Astérisque (1992) 235 MR1185803

[3] M Braverman, T Kappeler, Ray-Singer type theorem for the refined analytic torsion arXiv:math.GT/0603638

[4] M Braverman, T Kappeler, Refined analytic torsion arXiv:math.DG/0505537

[5] M Braverman, T Kappeler, Refined analytic torsion as an element of the determinant line arXiv:math.GT/0510532

[6] M Braverman, T Kappeler, A refinement of the Ray-Singer torsion, C. R. Math. Acad. Sci. Paris 341 (2005) 497-502 MR2180817

[7] D Burghelea, Removing metric anomalies from Ray-Singer torsion, Lett. Math. Phys. 47 (1999) 149-158 MR1682302

[8] D Burghelea, L Friedlander, T Kappeler, Relative torsion, Commun. Contemp. Math. 3 (2001) 15-85 MR1820014

[9] D Burghelea, S Haller, Complex valued Ray-Singer torsion arXiv: math.DG/0604484

[10] D Burghelea, S Haller, Dynamics, Laplace transform and spectral geometry arXiv: math.DG/0508216

[11] D Burghelea, S Haller, Torsion, as function on the space of representations arXiv: math.DG/0507587

[12] S Dineen, Complex analysis in locally convex spaces, North-Holland Mathematics Studies 57, North-Holland Publishing Co., Amsterdam (1981) MR640093Notas de Matemática [Mathematical Notes], 83 
[13] J Eells, Jr, A setting for global analysis, Bull. Amer. Math. Soc. 72 (1966) 751-807 MR0203742

[14] M Farber, Absolute torsion and eta-invariant, Math. Z. 234 (2000) 339-349 MR1765885

[15] M Farber, V Turaev, Poincaré-Reidemeister metric, Euler structures, and torsion, J. Reine Angew. Math. 520 (2000) 195-225 MR1748274

[16] D Fried, Lefschetz formulas for flows, from: "The Lefschetz centennial conference, Part III (Mexico City, 1984)", Contemp. Math. 58, Amer. Math. Soc., Providence, RI (1987) 19-69 MR893856

[17] P Griffiths, J Harris, Principles of algebraic geometry, Wiley-Interscience [John Wiley \& Sons], New York (1978) MR507725

[18] R Hartshorne, Algebraic geometry, Springer, New York (1977) MR0463157

[19] E Hille, R S Phillips, Functional analysis and semi-groups, American Mathematical Society Colloquium Publications, vol. 31, American Mathematical Society, Providence, R. I. (1957) MR0089373

[20] F Kamber, P Tondeur, Flat bundles and characteristic classes of grouprepresentations, Amer. J. Math. 89 (1967) 857-886 MR0220309

[21] A Kriegl, P W Michor, The convenient setting of global analysis, Mathematical Surveys and Monographs 53, American Mathematical Society, Providence, RI (1997) MR1471480

[22] J Marcsik, Analytic torsion and closed one forms, $\mathrm{PhD}$ thesis, Ohio State University (1995)

[23] V Mathai, D Quillen, Superconnections, Thom classes, and equivariant differential forms, Topology 25 (1986) 85-110 MR836726

[24] J Milnor, Whitehead torsion, Bull. Amer. Math. Soc. 72 (1966) 358-426 MR0196736

[25] L I Nicolaescu, The Reidemeister torsion of 3-manifolds, de Gruyter Studies in Mathematics 30, Walter de Gruyter \& Co., Berlin (2003) MR1968575

[26] D Quillen, Determinants of Cauchy-Riemann operators on Riemann surfaces, Funktsional. Anal. i Prilozhen. 19 (1985) 37-41, 96 MR783704

[27] J-P Ramis, Sous-ensembles analytiques d'une variété banachique complexe, Springer, Berlin (1970) MR0293126

[28] D B Ray, I M Singer, $R$-torsion and the Laplacian on Riemannian manifolds, Advances in Math. 7 (1971) 145-210 MR0295381

[29] N Steenrod, The topology of fibre bundles, Princeton Landmarks in Mathematics, Princeton University Press, Princeton, NJ (1999) MR1688579

[30] V G Turaev, Reidemeister torsion in knot theory, Uspekhi Mat. Nauk 41 (1986) 97-147, 240 MR832411 
[31] V G Turaev, Euler structures, nonsingular vector fields, and Reidemeister-type torsions, Izv. Akad. Nauk SSSR Ser. Mat. 53 (1989) 607-643, 672 MR1013714

[32] V Turaev, Introduction to combinatorial torsions, Lectures in Mathematics ETH Zürich, Birkhäuser Verlag, Basel (2001) MR1809561

[33] V Turaev, Torsions of 3-dimensional manifolds, Progress in Mathematics 208, Birkhäuser Verlag, Basel (2002) MR1958479

[34] G W Whitehead, Elements of homotopy theory, Graduate Texts in Mathematics 61, Springer, New York (1978) MR516508

Dept. of Mathematics, The Ohio State University

231 West 18th Avenue, Columbus, OH 43210, USA

Department of Mathematics, University of Vienna

Nordbergstrasse 15, A-1090, Vienna, Austria

burghele@mps.ohio-state.edu, stefan.haller@univie.ac.at

Proposed: Wolfgang Lück

Seconded: Bill Dwyer, Haynes Miller
Received: 16 December 2005

Revised: 27 March 2006 